\title{
Psychosocial interventions for benzodiazepine harmful use, abuse or dependence (Review)
}

Darker CD, Sweeney BP, Barry JM, Farrell MF, Donnelly-Swift E

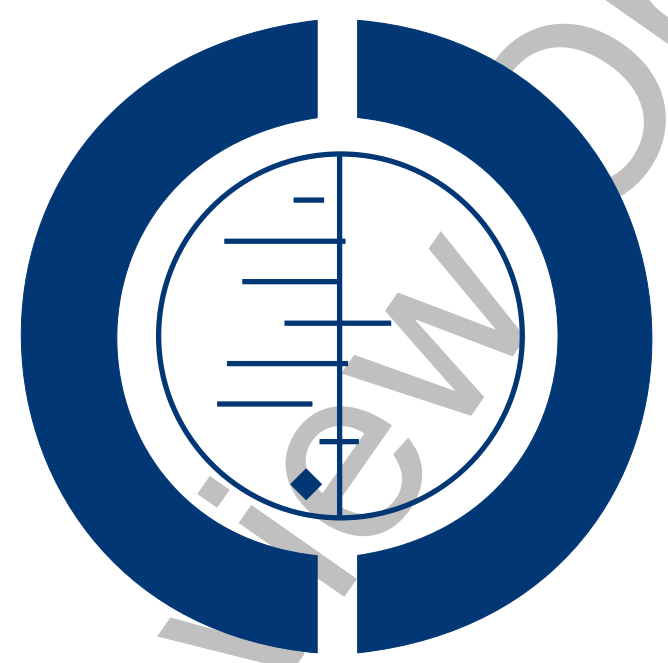

\section{THE COCHRANE COLLABORATION ${ }^{\circledR}$}

This is a reprint of a Cochrane review, prepared and maintained by The Cochrane Collaboration and published in The Cochrane Library 2015, Issue 5

http://www.thecochranelibrary.com

\section{WILEY}

Psychosocial interventions for benzodiazepine harmful use, abuse or dependence (Review)

Copyright @ 2015 The Cochrane Collaboration. Published by John Wiley \& Sons, Ltd. 
TABLE OF CONTENTS

HEADER

ABSTRACT . . . . . . . . . . . . . . . . . . . . . . . . . . . . . . . . . . . . . .

PLAIN LANGUAGE SUMMARY . . . . . . . . . . . . . . . . . . . . . . . . . . . . . . . .

SUMMARY OF FINDINGS FOR THE MAIN COMPARISON . . . . . . . . . . . . . . . . . . . . . . . 4

BACKGROUND . . . . . . . . . . . . . . . . . . . . . . . . . . . . . . . . . . . . . 6

OBJECTIVES . . . . . . . . . . . . . . . . . . . . . . . . . . . . . . . . . . . . . . . . . . . . . . . . . . .

METHODS . . . . . . . . . . . . . . . . . . . . . . . . . . . . . . . . . . . . . . 99

RESUlTS . . . . . . . . . . . . . . . . . . . . . . . . . . . . . . . . . . . . 12

Figure 1. . . . . . . . . . . . . . . . . . . . . . . . . . . . . . . . . . . 13

Figure 2. . . . . . . . . . . . . . . . . . . . . . . . . . . . . . . . . . 16

Figure 3. . . . . . . . . . . . . . . . . . . . . . . . . . . . . . . . . . . . . . 17

ADDITIONAL SUMMARY OF FINDINGS . . . . . . . . . . . . . . . . . . . . . . . . . . . $\quad$. 21

DISCUSSION . . . . . . . . . . . . . . . . . . . . . . . . . . . . . . . . . . . . . . . . .

AUTHORS' CONCLUSIONS . . . . . . . . . . . . . . . . . . . . . . . . . . . . . . . . . . .

ACKNOWLEDGEMENTS . . . . . . . . . . . . . . . . . . . . . . . . . . . . . . . . . 27

REFERENCES . . . . . . . . . . . . . . . . . . . . . . . . . . . . . . . . . . . . . . 27

CHARACTERISTICS OF STUDIES . . . . . . . . . . . . . . . . . . . . . . . . . . . . . . . . . . . . . .

DATA AND ANALYSES . . . . . . . . . . . . . . . . . . . . . . . . . . . . . . . . . . . . . . . . . . . . . . . . . . .

Analysis 1.1. Comparison 1 CBT (plus taper) versus taper, Outcome 1 Successful discontinuation of BZDs. $\quad$. $\quad$. $\quad$. 77

Analysis 1.2. Comparison 1 CBT (plus taper) versus taper, Outcome 2 Sensitivity analysis (Allocation concealment): successful discontinuation of BZDs.

Analysis 1.3. Comparison 1 CBT (plus taper) versus taper, Outcome 3 Sensitivity analysis (Blinding of assessor): successful discontinuation of BZDs. . . . . . . . . . . . . . . . . . . . . . . . . . . . . . . . . . . 80

Analysis 1.4. Comparison 1 CBT (plus taper) versus taper, Outcome 4 Reduce BZDs $>50 \%$. . . . . . . . . . . . . 81

Analysis 1.5. Comparison 1 CBT (plus taper) versus taper, Outcome 5 Drop-outs or lost to follow-up. . . . . . . $\quad 83$

Analysis 2.1. Comparison 2 MI versus TAU, Outcome 1 Successful discontinuation of BZDs. . . . . . . . . . . 84

Analysis 2.2. Comparison 2 MI versus TAU, Outcome 2 Reduce BZD $>50 \%$. . . . . . . . . . . . . . . . . . . . . 85

Analysis 3.1. Comparison 3 Standardised interview (taper) versus TAU, Outcome 1 Successful discontinuation of BZDs. 86

Analysis 3.2. Comparison 3 Standardised interview (taper) versus TAU, Outcome 2 Reduce BZD > 50\%. . . . . . 87

Analysis 4.1. Comparison 4 CBT (no taper) versus TAU, Outcome 1 BZD positive urine rate. . . . . . . . . . . . 88

Analysis 5.1. Comparison 5 Self-help booklet plus CBT versus self-help booklet, Outcome 1 Change in OTI score for BZD use.

Analysis 6.1. Comparison 6 Complaints management (additional relaxation) versus anxiety management (relaxation),

Outcome 1 Successful discontinuation of BZDs. . . . . . . . . . . . . . . . . . . . . . . . . . . . . . 90

Analysis 7.1. Comparison 7 Consultation (plus letter) versus TAU, Outcome 1 Successful discontinuation of BZD. . 91

Analysis 8.1. Comparison 8 E-counselling versus onsite counselling, Outcome 1 Positive BZD urine toxicology. . . . 91

Analysis 9.1. Comparison 9 Relaxation versus TAU, Outcome 1 Successful discontinuation of BZDs. . . . . . . . . 92

Analysis 10.1. Comparison 10 Tailored letter versus GP letter, Outcome 1 Successful discontinuation of BZDs. $\quad . \quad$. 93

Analysis 11.1. Comparison 11 Relaxation (plus taper) versus taper, Outcome 1 Successful discontinuation of BZDs. . 94

Analysis 12.1. Comparison 12 Enhanced skills training (relaxation) versus limited skills training (relaxation), Outcome 1

Change in prescribed diazepam dose $(\mathrm{mg}) . \quad$. . . . . . . . . . . . . . . . . . . . . . . . . . 95

APPENDICES . . . . . . . . . . . . . . . . . . . . . . . . . . . . . . . . . . . . . 95

CONTRIBUTIONS OF AUTHORS . . . . . . . . . . . . . . . . . . . . . . . . . . . . . . . . . . . . . 107

DECLARATIONS OF INTEREST . . . . . . . . . . . . . . . . . . . . . . . . . . . . . . . . . . . . . . . . .

SOURCES OF SUPPORT . . . . . . . . . . . . . . . . . . . . . . . . . . . . . . . . . . . . . . . . . . . . . 108

DIFFERENCES BETWEEN PROTOCOL AND REVIEW . . . . . . . . . . . . . . . . . . . . . . . . 108

Psychosocial interventions for benzodiazepine harmful use, abuse or dependence (Review)

Copyright $\odot 2015$ The Cochrane Collaboration. Published by John Wiley \& Sons, Ltd. 


\title{
[Intervention Review]
}

\section{Psychosocial interventions for benzodiazepine harmful use, abuse or dependence}

\author{
Catherine D Darker ${ }^{1}$, Brion P Sweeney ${ }^{2}$, Joe M Barry ${ }^{1}$, Michael F Farrell ${ }^{3}$, Erica Donnelly-Swift ${ }^{4}$ \\ ${ }^{1}$ Department of Public Health \& Primary Care, Trinity College Dublin, Dublin, Ireland. ${ }^{2}$ Health Service Executive Addiction Service, \\ Dublin, Ireland. ${ }^{3}$ National Drug and Alcohol Research Centre, University of New South Wales, Sydney, Australia. ${ }^{4}$ Trinity College \\ Dublin, Dublin, Ireland
}

Contact address: Catherine D Darker, Department of Public Health \& Primary Care, Trinity College Dublin, Adelaide \& Meath Hospital Dublin, Incorporating the National Childrens Hospital, Tallaght, Dublin, D24, Ireland. catherine.darker@tcd.ie.

Editorial group: Cochrane Drugs and Alcohol Group.

Publication status and date: New, published in Issue 5, 2015.

Review content assessed as up-to-date: 9 December 2014.

Citation: Darker CD, Sweeney BP, Barry JM, Farrell MF, Donnelly-Swift E. Psychosocial interventions for benzodiazepine harmful use, abuse or dependence. Cochrane Database of Systematic Reviews 2015, Issue 5. Art. No.: CD009652. DOI: 10.1002/14651858.CD009652.pub2.

Copyright (C) 2015 The Cochrane Collaboration. Published by John Wiley \& Sons, Ltd.

\section{Background}

\section{A B S T R A C T}

Benzodiazepines (BZDs) have a sedative and hypnotic effect upon people. Short term use can be beneficial but long term BZD use is common, with several risks in addition to the potential for dependence in both opiate and non-opiate dependent patients.

\section{Objectives}

To evaluate the effectiveness of psychosocial interventions for treating BZD harmful use, abuse or dependence compared to pharmacological interventions, no intervention, placebo or a different psychosocial intervention on reducing the use of BZDs in opiate dependent and non-opiate dependent groups.

\section{Search methods}

We searched the Cochrane Central Register of Controlled Trials (CENTRAL- the Cochrane Library issue 12, 2014) which includes the Cochrane Drugs and Alcohol Group Specialized Register; PubMed (from 1966 to December 2014); EMBASE (from 1988 to December 2014); CINAHL Cumulative Index to Nursing and Allied Health Literature (1982 to September 2013 ); PsychINFO (1872 to December 2014); ERIC (Education Resources Information Centre, (January 1966 to September 2013); All EBM Reviews (1991 to September 2013, Ovid Interface); AMED (Allied \& Alternative Medicine) 1985 to September 2013); ASSIA (Applied Social Sciences Index \& Abstracts (1960 to September 2013); LILACS (January 1982 to September 2013); Web of Science (1900 to December 2014); Electronic Grey Literature Databases: Dissertation Abstract; Index to Theses.

\section{Selection criteria}

Randomised controlled trials examining the use of a psychosocial intervention to treat BZDs versus pharmacological interventions, no intervention, placebo or a different psychosocial intervention on reducing the use of BZDs in opiate dependent and non-opiate dependent groups.

\section{Data collection and analysis}

We used the standard methodological procedures outlined in Cochrane Guidelines.

Psychosocial interventions for benzodiazepine harmful use, abuse or dependence (Review)

Copyright (๑) 2015 The Cochrane Collaboration. Published by John Wiley \& Sons, Ltd. 


\section{Main results}

Twenty-five studies including 1666 people met the inclusion criteria. The studies tested many different psychosocial interventions including cognitive behavioural therapy (CBT) (some studies with taper, other studies with no taper), motivational interviewing (MI), letters to patients advising them to reduce or quit BZD use, relaxation studies, counselling delivered electronically and advice provided by a general practitioner (GP). Based on the data obtained, we performed two meta-analyses in this Cochrane review: one assessing the effectiveness of CBT plus taper versus taper only (575 participants), and one assessing MI versus treatment as usual (TAU) (80 participants).

There was moderate quality of evidence that CBT plus taper was more likely to result in successful discontinuation of BZDs within four weeks post treatment compared to taper only (Risk ratio (RR) 1.40, 95\% confidence interval (CI) 1.05 to 1.86 ; nine trials, 423 participants) and moderate quality of evidence at three month follow-up (RR 1.51, 95\% CI 1.15 to 1.98) in favour of CBT (taper) for 575 participants. The effects were less certain at 6, 11, 12, 15 and 24 months follow-up. The effect of CBT on reducing BZDs by $>50 \%$ was uncertain for all time points examined due to the low quality evidence. There was very low quality evidence for the effect on drop-outs at any of the time intervals; post-treatment (RR 1.05, 95\% CI 0.66 to 1.66), three month follow-up (RR $1.71,95 \%$ CI 0.16 to 17.98 ) and six month follow-up (RR $0.70,95 \%$ CI 0.17 to 2.88 ).

Based on the very low quality of evidence available, the effect of MI versus TAU for all the time intervals is unclear; post treatment (RR 4.43, 95\% CI 0.16 to 125.35; two trials, 34 participants), at three month follow-up (RR 3.46, 95\% CI 0.53 to 22.45; four trials, 80 participants), six month follow-up (RR $0.14,95 \%$ CI 0.01 to 1.89 ) and 12 month follow-up (RR 1.25 , 95\% CI 0.63 to 2.47 ). There was very low quality of evidence to determine the effect of MI on reducing BZDs by $>50 \%$ at three month follow-up (RR 1.52 , 95\% CI 0.60 to 3.83) and 12 month follow-up (RR 0.87, 95\% CI 0.52 to 1.47). The effects on drop-outs from treatment at any of the time intervals between the two groups were uncertain due to the wide CIs; post-treatment (RR 0.50, 95\% CI 0.04 to 7.10), three month follow-up (RR 0.46, 95\% CI 0.06 to 3.28), six month follow-up (RR 8.75, 95\% CI 0.61 to 124.53 ) and 12 month follow-up (RR $0.42,95 \%$ CI 0.02 to 7.71$)$.

The following interventions reduced BZD use - tailored GP letter versus generic GP letter at 12 month follow-up (RR 1.70, 95\% CI 1.07 to 2.70; one trial, 322 participants), standardised interview versus TAU at six month follow-up (RR 13.11, 95\% CI 3.25 to 52.83; one trial, 139 participants) and 12 month follow-up (RR 4.97, 95\% CI 2.23 to 11.11), and relaxation versus TAU at three month follow-up (RR 2.20, $95 \%$ CI 1.23 to 3.94 ).

There was insufficient supporting evidence for the remaining interventions.

We performed a 'Risk of bias' assessment on all included studies. We assessed the quality of the evidence as high quality for random sequence generation, attrition bias and reporting bias; moderate quality for allocation concealment, performance bias for objective outcomes, and detection bias for objective outcomes; and low quality for performance bias for subjective outcomes and detection bias for subjective outcomes. Few studies had manualised sessions or independent tests of treatment fidelity; most follow-up periods were less than 12 months.

Based on decisions made during the implementation of protocol methods to present a manageable summary of the evidence we did not collect data on quality of life, self-harm or adverse events.

\section{Authors' conclusions}

CBT plus taper is effective in the short term (three month time period) in reducing BZD use. However, this is not sustained at six months and subsequently. Currently there is insufficient evidence to support the use of MI to reduce BZD use. There is emerging evidence to suggest that a tailored GP letter versus a generic GP letter, a standardised interview versus TAU, and relaxation versus TAU could be effective for BZD reduction. There is currently insufficient evidence for other approaches to reduce BZD use.

\section{PLAIN LANGUAGE SUMMARY}

Psychosocial interventions to reduce sedative use, abuse and dependence

\section{Background}

In this Cochrane review we aimed to measure the effectiveness of psychosocial interventions for treating people who harmfully use, abuse or are dependent on benzodiazepines (BZDs). BZDs are a type of drug that can be used to treat people who have anxiety, panic 
disorder, insomnia and a range of other conditions. Long term use of BZDs is not generally recommended and can lead to physical and psychological dependence and withdrawal symptoms when patients reduce or stop using them. Previous systematic reviews, examining other drugs like heroin, cocaine or alcohol, have suggested some benefits of psychosocial interventions to reduce these substances. There has been no Cochrane review of psychosocial interventions to reduce BZD use.

\section{Study characteristics}

We searched electronic databases and did handsearches to identify and report on all studies (up to December 2014) where participants were randomly assigned to active treatment with a psychosocial intervention or to a control group of no intervention or treatment as usual (TAU). We included 25 studies with 1666 participants in total that fulfilled these criteria. Two psychosocial methods, in particular cognitive behavioural therapy (CBT) (11 studies, 575 participants) and motivational interviewing (MI) (4 studies, 80 participants) were of high enough quality and sufficiently similar to one another to perform meta-analyses. We did not subject the other included studies (10 studies, 1042 participants) to meta-analysis. These smaller studies used a range of approaches including: a tailored letter and standardised interview between patients with their prescribing general practitioner (GP) and relaxation techniques.

\section{Key findings}

We found that CBT studies showed a short term benefit when added to taper but this benefit was not sustained beyond three months. MI studies did not support the use of MI to reduce BZD use.

Three smaller studies showed some promise. One trial showed that tailored letters sent by GPs to patients versus standard GP letter encouraged patients to cease or reduce their BZD use (one trial, 322 participants) where there was evidence in favour of tailored letter (twice as likely) to cease BZD use at 12 months follow-up. A study with 139 participants which compared standardised interview plus taper versus TAU and showed evidence of benefit in both discontinuation and reduction of BZDs at six and 12 months, but not 36 months. One relaxation study, with 60 participants, comparing relaxation versus TAU was significant at three-month follow-up for the successful discontinuation of BZDs.

Other studies using a variety of interventions including self help booklet, e-counselling, self help booklet plus minimal dose of CBT or CBT without taper did not show a benefit in reducing BZD use.

Based on decisions made during the implementation of protocol methods to present a manageable summary of the evidence we did not collect data on quality of life, self-harm or adverse events.

\section{Quality of evidence}

We downgraded the quality of the evidence for many of the outcomes in this review. Some studies relied almost entirely on patients self report to clinicians which is not a very reliable way of measuring outcomes, especially in substance misuse research. Most studies involved small numbers of participants, and there was some inconsistency in the findings. In addition, many of the smaller studies were potentially confounded by having poorly defined control groups; e.g. advanced skills training in symptom management versus limited skills training or in another study anxiety management plus relaxation versus relaxation alone or e-counselling versus onsite counselling in a clinic.

\section{Conclusion}

CBT plus taper is effective in the short term (three month time period) in reducing BZD use. However, this is not maintained at six months and subsequently. The possibility of including a 'top-up' of CBT to sustain long term effects should be investigated. Currently there is insufficient evidence to support the use of MI to reduce BZD use. There is some evidence to suggest that a tailored GP letter versus a general GP letter, standardised interview versus TAU and relaxation versus TAU could be effective for BZD reduction. There is currently insufficient evidence for other psychosocial approaches to reduce BZD use.

Psychosocial interventions for benzodiazepine harmful use, abuse or dependence (Review) 


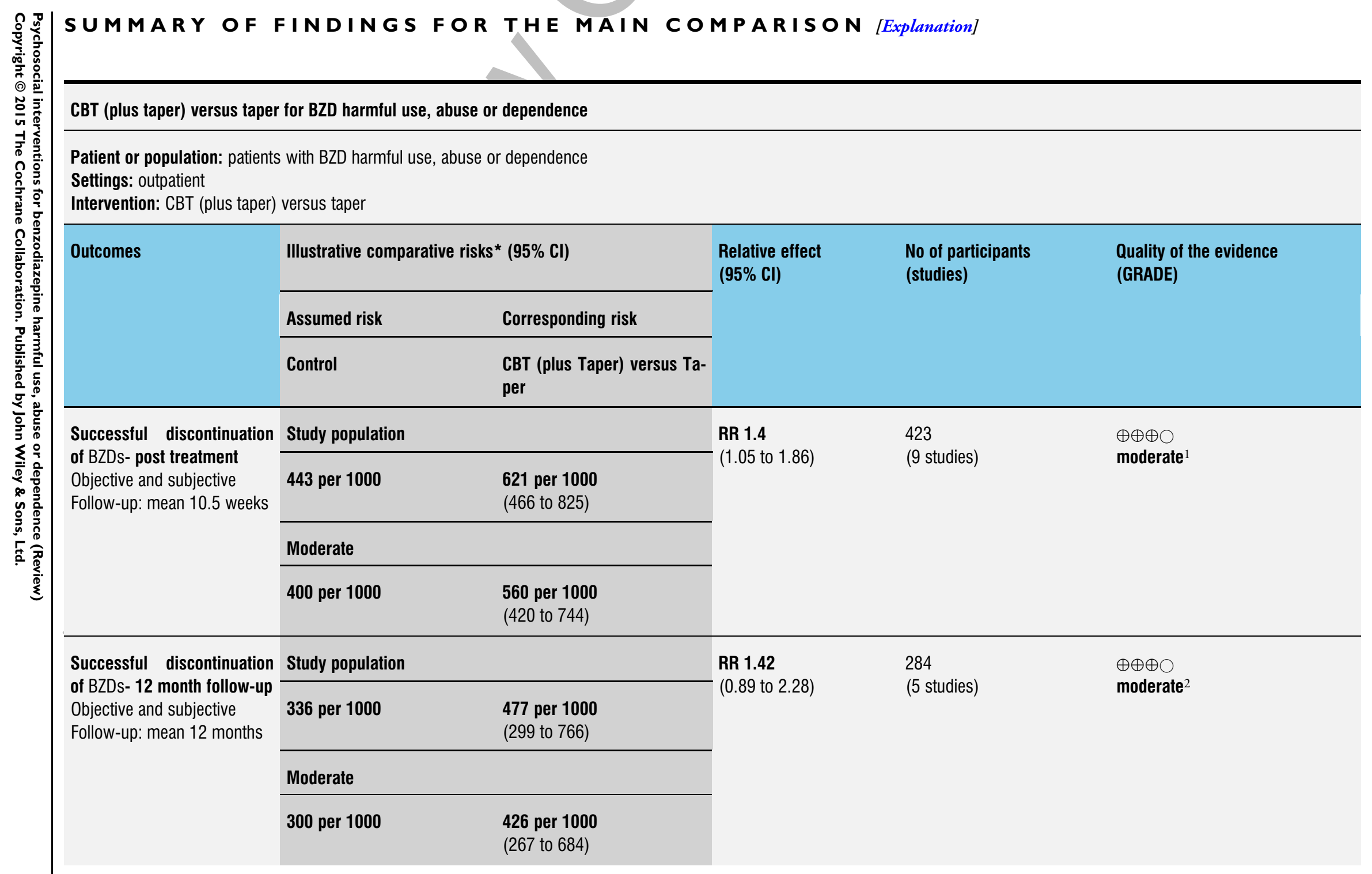




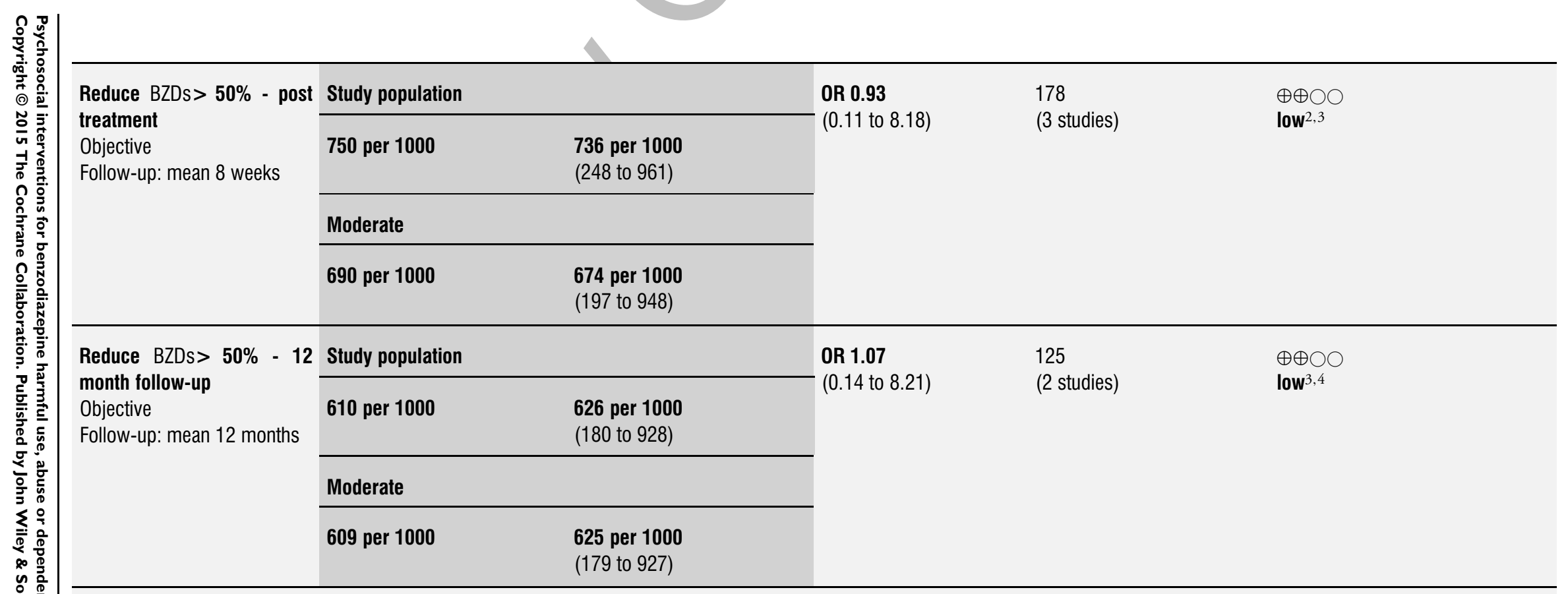

*The basis for the assumed risk (e.g. the median control group risk across studies) is provided in footnotes. The corresponding risk (and its $95 \% \mathrm{Cl}$ ) is based on the assumed risk in the comparison group and the relative effect of the intervention (and its $95 \% \mathrm{Cl}$ ).

Cl: confidence interval; RR: risk ratio; OR: odds ratio; BZD: benzodiazepine; CBT: cognitive behavioural therapy.

\section{GRADE Working Group grades of evidence}

High quality: Further research is very unlikely to change our confidence in the estimate of effect.

Moderate quality: Further research is likely to have an important impact on our confidence in the estimate of effect and may change the estimate.

Low quality: Further research is very likely to have an important impact on our confidence in the estimate of effect and is likely to change the estimate.

Very low quality: We are very uncertain about the estimate.

'Downgraded by one level due to serious risk of bias. Five studies were at high risk of detection bias; one study was also at high risk of attrition bias and another one at high risk of detection bias.

${ }^{2}$ Downgraded by one level due to serious risk of bias. Two studies were at high risk of detection bias and one study each was also at high risk of attrition and selection bias.

${ }^{3}$ Downgraded by one level due to serious imprecision (wide Cls).

${ }^{4}$ Downgraded by one level due to serious risk of bias. One study was at high risk of detection and attrition bias. 


\section{B A C K G RO U N D}

Benzodiazepines (BZDs) enhance the effects of the major inhibitory neurotransmitter gamma-aminobutyric acid (GABA) in the central nervous system (CNS) (Oliver 2007). Slowing down the CNS has a range of effects, including inducing sleep, causing sedation, reducing anxiety and panic and relaxing muscles. BZDs are used mainly as sedatives or hypnotics, muscle relaxants, and anti-epileptics, and were once referred to by the now-deprecated term "tranquillisers" (WHO 2010). BZDs are widely prescribed for the treatment of anxiety and insomnia. While they can initially be helpful in relieving the symptoms of these problems, many people develop a tolerance to their effects, gain little therapeutic benefit from chronic consumption, become dependent on them and suffer a withdrawal syndrome when they stop taking them. The withdrawal syndrome may be prolonged and can develop at any time up to three weeks after cessation of a long-acting BZD, or a few hours after cessation of a short-acting one. The syndrome includes rebound anxiety, depression, nausea, perceptual changes and even epileptic seizures and psychosis in rare instances. Some people may intentionally abuse BZDs. The individuals who intentionally abuse BZDs and those who inadvertently become dependent on them may differ substantially in clinical and demographic characteristics and possibly in response to treatment. Misuse of BZDs is most often found within a polydrug use pattern (the use of two or more psychoactive drugs in combination to achieve a particular effect) as an attempt to achieve a subjective euphoria or reduce anxiety symptoms or treat the side or withdrawal effects of other drugs of abuse (WHO 2010).

\section{Description of the condition}

BZDs are widely prescribed for the treatment of people with anxiety and insomnia. While BZDs can initially be helpful in relieving the symptoms of these problems they carry a risk of dependence and withdrawal. Long-term use of BZDs have recently been associated with dementia (Billioti de Gage 2012), and impaired cognitive attention (Petursson 1993) and verbal memory (Barker 2004), increased risk of road traffic accidents (Smink 2010), hip fractures (Wagner 2004), and falls (Bartlett 2009) in the elderly. Some people, such as those dependent on opiates, may concomitantly use BZDs as a way of augmenting the effects of the opiates. BZD use in people dependent on opioids is correlated with a history of more severe drug abuse (Chutuape 1997; Darke 1993), a high level of psychological distress (Bleich 1999), more HIV risktaking behaviours (Darke 1994) and a higher prevalence of hepatitis C virus (HCV) infection (van den Hoek 1990). The synergistic effect of BZD and opiate use increases the risk of overdose due to a synergistic depressant effect on the respiratory system (Jones 2012).

\section{Harmful use}

The World Health Organization (WHO) has characterised harmful use as:

- A pattern of psychoactive substance use that is causing damage to the mental (e.g. episodes of depressive disorder secondary to heavy consumption of alcohol) or physical health (e.g. in cases of hepatitis from the self-administration of injected drugs) of the user (WHO 2009a).

\section{Abuse}

Substance abuse is defined by the American Psychiatric Association (DSM-IV 1994) as a maladaptive pattern of substance use leading to clinically significant impairment or distress as manifested by one (or more) of the following, occurring within a 12-month period:

- Recurrent BZD use resulting in a failure to fulfil major role obligations at work, school or home.

- Recurrent BZD use in situations in which it is physically hazardous.

- Recurrent BZD related legal problems.

- Continued BZD use despite having persistent or recurrent social or interpersonal problems caused or exacerbated by the effects of BZDs.

\section{Dependence}

Dependence is characterised by the International Classification of Diseases and Related Health Problems, WHO as a cluster of physiological, behavioural, and cognitive phenomena as manifested by three (or more) of the following, occurring within a 12-month period (WHO 2009a):

- A strong desire or sense of compulsion to take BZDs.

- Difficulties in controlling BZD consumption in terms of its onset, termination, or levels of use.

- A physiological withdrawal when BZD use has ceased or has been reduced.

- Evidence of tolerance, such that increased doses of BZDs are required in order to achieve effects originally produced by lower doses.

- Progressive neglect of alternative pleasures or interests because of BZD use, increased amount of time necessary to obtain or take the substance or to recover from its effects.

- Persisting with BZD use despite clear evidence of overtly harmful consequences.

\section{Misuse and aberrant abuse or dependence}

Misuse of BZDs occurs in people who have been prescribed BZDs to treat underlying conditions such as sleep, anxiety or panic disorders. Aberrant use can occur in patients who begin using BZDs to treat a diagnosed disorder and end up using them inappropriately. Individuals who abuse prescribed BZDs take them in higher 
doses than their prescribing doctor intended or for a longer duration than needed after remission of the condition for which they were prescribed BZDs, to enhance the effects of other drugs or to reduce withdrawal symptoms and who then may progress to dependence. Some people may also use BZDs in very high doses or may inject or obtain them illicitly. Within this constituency, BZDs may also be used to self-medicate the withdrawal effects of other substances. For example, methadone maintained opiate dependent patients may take a BZD after taking methadone to augment the subjective effects they experience by increasing sedation. Individuals who are opiate dependent can co-inject BZDs with opiates to intensify the effect and in both instances are also cotreating their BZD dependence (Backmund 2005). BZDs are also used to self-medicate the symptoms of opiate withdrawal or treat the adverse effects of other drugs like cocaine or alcohol (O'Brien 2005).

People who abuse BZDs may become dependent on them, although abuse and dependency are not always mutually exclusive. Some patients' use of BZDs may progress to dependence. During therapeutic use, the risk of developing BZD dependence increases with the dose and duration of treatment, the nature of the illness, the severity of symptoms, the expectation of beneficial effect and the intensity of stress factors. Long term use of BZDs for the treatment of generalised anxiety disorder is now contraindicated (British National Formulary 2010; NICE 2011).

Dependence on BZDs has been recognised as a significant clinical problem for over 30 years (Tyrer 2010). Dependence is now recognised as a significant risk in patients receiving BZD treatment for longer than one month (Department of Health \& Children 2002).

\section{Description of the intervention}

For the purposes of this Cochrane review, we defined psychosocial interventions to include "any non-pharmacological intervention carried out in a therapeutic context at an individual, family or group level" (WHO 2009b). We included any psychosocial intervention as long as they were validated or described by the trial author(s), allowing reproduction. There can be a wide range of psychosocial interventions that target BZD abuse or dependence. Hence, this Cochrane review is comprehensive in the list of interventions which were considered with the aim of including every type of psychosocial intervention provided to patients.

The most commonly used approaches are:

- Cognitive behavioural therapy (CBT) is a discrete, timelimited, structured psychological intervention, derived from a cognitive model of drug misuse (Beck 1993). There is an emphasis on identifying and modifying irrational thoughts, managing negative mood and intervening after a lapse to prevent a full-blown relapse. CBT in addiction is based on the principle that addictions are learned behaviours that are capable of being modified. Cognitive approaches primarily aim to change addictive behaviour through changes in faulty cognitions (e.g. dysfunctional beliefs) that serve to maintain the behaviour, or through the promotion of positive cognitions (e.g. self efficacy) or motivation to change behaviour. Behavioural approaches primarily aim to modify behaviours underpinned by conditioned learning: classical and operant conditioning. Such approaches are many and varied, but include interventions aimed at extinguishing classically conditioned responding (e.g. cue exposure and response prevention) whereby patients are helped deal with stimuli (triggers) that lead to relapse such as external cues (sight of your dealer) to internal cues, such as mood states.

- In the motivational approach (motivational interviewing (MI), motivational enhancement therapy) rather than confront the patient's resistance to abstinence in a direct and sometimes confrontational manner, the therapist "rolls with resistance" and tries to help the patient develop more self-motivation to stop using via specified techniques (Miller 1991).

- Brief interventions (BIs) are time limited, structured and directed toward a specific goal (SAMHSA 1999). There is much recent interest in Screening, Brief Intervention and Referral To Treatment (SAMHSA 2014). Definitions of BIs vary and in recent literature have been referred to as "simple advice", "minimal interventions", "brief counselling" or "short-term counselling". They can be simple suggestions to reduce substance use given by a professional (e.g. social worker, nurse, counsellor, doctor) or a series of interventions provided within a treatment programme. They can follow a specific plan (and in some cases a workbook) and have timelines for the adoption of specific behaviours.

- Contingency management considers drug use as an example of operant behaviour that is maintained partly by the pharmacological effects of the drug in combination with other social and non-drug reinforcement provided by the drug using lifestyle (Stitzer 2006). Contingency management uses positive and negative contingencies to enhance motivation whereby substance use may lead to a loss of reinforcement (often monetary reward), while abstinence leads to positive reinforcement.

- Drug counselling includes a strong emphasis on abstinence, and assistance with social, family and legal problems. It focuses on behaviours and external events rather than intrapsychic processes (Onken 1990).

- The 12-step approach is a self help approach based on a set of guiding principles outlining a course of action for recovery from addiction, compulsion or other behavioural problems. Originally proposed by Alcoholics Anonymous as a method of recovery from alcoholism, the method was then adapted and became the foundation of other 12-step programmes such as Narcotics Anonymous. Members are encouraged to regularly attend meetings with other members who share their particular recovery philosophy. 
- Psychotherapy includes many different approaches and is based on the concept that psychiatric disorders, including substance addiction, are intimately associated with disturbances in intrapersonal and interpersonal functioning, which may be associated with the genesis and perpetuation of the disorder (Rounsaville 1983). Supportive expressive techniques aim to help the participant feel comfortable in discussing his or her personal experiences. The expressive techniques aim to help the participant identify and work through problematic relationship themes. Special attention is paid to themes that are involved in drug dependence, the role of drugs in relation to problem feelings and behaviours and how problems may be solved without recourse to drugs. Short-term psychodynamic interventions are derived from a psychodynamic/psychoanalytic model in which: a) therapist and patient explore and gain insight into conflicts and how these are represented in current situations and relationships, including the therapy relationship by exploring transference issues in a very direct way; b) patients are given an opportunity to explore feelings and conscious and unconscious conflicts originating in the past, with the technical focus on interpreting and working through conflicts; c) therapy is non-directive and patients are not taught specific skills such as thought monitoring, re-evaluation or problem solving. Treatment typically consists of 16 to 30 sessions.

- A long term residential approach views substance use as a disorder of the whole person, involving the possibility of impeded personality development with concomitant deficits in social, educational and economic/survival skills. This global perspective of the problem recommends a multidimensional rehabilitative approach that occurs in a 24-hour residential setting removing a person from an ongoing unmanageable and sometime dangerous community setting (Brunette 2004).

- Social behaviour and network therapy (SBNT) is built upon the premise that social network support for change is central to the resolution of addictive behaviour (UKATT 2001). Wherever possible, SBNT engages families and friends of the person with the addiction problem in the treatment process in order to mobilise and develop social network support for change of the addictive behaviour.

- The community-reinforcement approach (CRA) is a treatment approach that aims to achieve abstinence by eliminating positive reinforcement of drug taking and enhancing positive reinforcement for sobriety. CRA integrates several treatment components, including building the patient's motivation to quit, helping the patient initiate sobriety, analysing the patients' drug and drinking pattern, increasing positive reinforcement, learning new coping behaviours, and involving significant others in the recovery process. In community reinforcement emphasis is placed on environmental contingencies in aspects of life such as work, recreation, family involvement and so on, to promote a lifestyle that is more rewarding than drug misuse (Miller 1999).

- Relapse prevention (Marlatt 1985) places emphasis on training people who misuse drugs to develop skills to identify situations or states where they are most vulnerable to drug use, to avoid high-risk situations and to use a range of cognitive and behavioural strategies to cope effectively with these situations (Carroll 1996). Relapse prevention strategies also target the person's lifestyle and the rewards they get from ordinary tasks of living and encourage an increase in life enhancing lifestyles.

- Couples-based interventions involve the spouse or partner expressing active support for the person who uses drugs in reducing drug use, including via the use of behavioural contracts. Couples are helped to improve their relationship through more effective communication skills and encouraged to increase positive behavioural exchanges through acknowledgement of pleasing behaviours and engagement in shared recreational activities (Fals-Stewart 2005).

\section{How the intervention might work}

Psychosocial interventions vary depending on the theoretical model underpinning them and can have a number of aims, such as:

- Facilitate the withdrawal itself.

- Treat or modify any underlying disorder or comorbidity that either complicates the addictive disorder or acts as a trigger for relapse.

- Generate and encourage alternative behaviours based on rewards.

- Modify underlying unconscious dynamic aspects.

- Work directly with cognitions that lead to substance misuse.

- Work with conditioned and operant response.

- Encourage engagement with pharmacotherapy.

- Maintain abstinence over time.

Clearly the contexts in which these different approaches are used will vary. Approaches to modify addictive behaviour can be used in any treatment context either as an adjunct to pharmacotherapy or as the primary treatment intervention. Psychosocial treatments to enhance compliance with pharmacotherapy are context specific. Psychosocial interventions to treat psychiatric comorbidity are clearly targeted at subgroups of addicted individuals with specific comorbidities. However, the literature on psychosocial interventions is often unclear regarding what is the specific aim of the therapy or the specific comorbidities of the patient group.

\section{Why it is important to do this review}

The Cochrane Drugs and Alcohol Group (CDAG) has conducted nine reviews of psychosocial interventions on a range of substances, 
such as opioids (Amato 2011a; Amato 2011b; Mayet 2005) alcohol (Ferri 2006; Kaner 2007; Lui 2008; McQueen 2009) and cocaine (Denis 2006; Knapp 2007). Some trials included in these Cochrane reviews have suggested that psychosocial interventions can be effective in reducing substance abuse and dependence. However, there has never been a review of the evidence for psychosocial interventions for the treatment of BZD harmful use, abuse or dependence.

Overall the psychosocial component of therapy is thought to be a critical component of the holistic treatment and is delivered in various ways in different countries and across a range of treatment settings. What is striking is the heterogeneous range of psychosocial interventions that are provided in the field of drug abuse and dependence. This heterogeneity makes comparison of psychosocial interventions a significant challenge across the field of substance misuse research. It remains unclear if psychosocial treatments are effective for the treatment of BZD harmful use, abuse or dependence and which intervention is most effective.

Evidence from randomised controlled trials (RCTs) on the subject of this topic needs to be summarised.

\section{O B J E C T I VES}

To assess the effectiveness of psychosocial interventions for treating BZD harmful use, abuse or dependence compared to pharmacological interventions, no intervention, placebo or a different psychosocial intervention on reducing use of BZDs in opiate dependent and non-opiate dependent groups.

\section{METHODS}

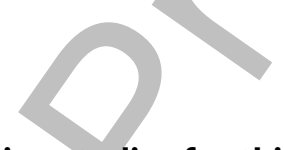

\section{Criteria for considering studies for this review}

\section{Types of studies}

RCTs evaluating the effectiveness of psychosocial interventions for BZD harmful use, abuse or dependence within opiate dependent populations and non-opiate dependent populations. We included trials undertaken in residential and outpatient facilities in primary and secondary care settings.

\section{Types of participants}

Opiate dependent populations and non-opiate dependent populations.

Exclusion criteria:

- People 15 years of age or younger.

Inclusion criteria:
- People with a dual diagnosis. The WHO has described dual diagnosis as a general term referring to comorbidity or the cooccurrence in the same individual suffering from both a substance problem and another mental health issue such as depression or an anxiety disorder (WHO 2010). Dual diagnosis can occur within an opiate dependent and a non-opiate dependent population.

\section{Types of interventions}

We included any psychosocial intervention as long as it was validated or described by the study author(s), allowing reproduction. Psychosocial interventions are defined to include "any non-pharmacological intervention carried out in a therapeutic context at an individual, family or group level" (WHO 2009b).

The intervention group should not have included any pharmacotherapy and could include interventions such as:

contingency management, community reinforcement approaches, CBTs, relapse prevention, couples based interventions, familybased interventions, psychodynamic therapies, drug abuse counselling, BIs, coping skills training, supportive expressive therapy, social skills training, stress management, relaxation therapy, relapse prevention, dialectical behavioural therapy, MI or motivational enhancement therapies.

Comparisons:

- Pharmacotherapy alone.

- No intervention (untreated control groups; usual care; waiting list controls).

- Placebo or sham method.

- A different psychosocial intervention.

\section{Types of outcome measures}

We only included validated measures for all outcomes.

\section{Primary outcomes}

We were particularly interested in reduction of BZD use classified as either successful discontinuation of BZD use or reduction of BZD use by $>50 \%$.

Use of BZDs at the end of treatment was measured by:

- Any biological marker of BZD metabolites provided in original studies (e.g. urine drug screen or hair analysis).

- Self-reported use of BZDs.

- Degree of effective dose reduction (e.g. frequency of BZD intake).

- Abstinence rates.

- Time to relapse.

- Drop-outs/loss to follow-up.

Secondary outcomes

Psychosocial interventions for benzodiazepine harmful use, abuse or dependence (Review) 
In this Cochrane review we adopted a very broad approach, both in terms of defining an intervention and picking a condition/diagnosis to examine. Because of these factors we already had a degree of heterogeneity and analysis of secondary outcomes would have given rise to a less accessible and intelligible review. Concentrating on the primary outcome gives a set of messages that are more clinically relevant and useful.

\section{Search methods for identification of studies}

- Electronic searches of databases.

Other sources of literature:

- Grey literature.

- Handsearching.

- References lists.

- Personal communication.

- Institutional repositories.

\section{Electronic searches}

We obtained relevant trials by searching the following sources:

- Electronic bibliographic databases:

1. Cochrane Central Register of Controlled Trials (CENTRAL- the Cochrane Library, Issue 12, 2014) which include the CDAG Specialized Register (Appendix 1).

2. PubMed (from 1966 to December 2014) (Appendix 2).

3. EMBASE (from 1988 to December 2014) (Appendix 3).

4. CINAHL Cumulative Index to Nursing and Allied Health Literature (1982 to September 2013) (Appendix 4).

5. PsychINFO (1872 to December 2014) (Appendix 5).

6. ERIC (Education Resources Information Centre, (January 1966 to September 2013) (Appendix 6).

7. All EBM Reviews (1991 to September 2013, Ovid Interface) (Appendix 7).

8. AMED (Allied \& Complementary Medicine) 1985 to September 2013) (Appendix 8).

9. ASSIA (Applied Social Sciences Index \& Abstracts (1960 to September 2013) (Appendix 9).

10. LILACS (Jan 1982 to September 2013) (Appendix 10).

11. Web of Science (1900 to December 2014) (Appendix 11).

12. National Register (1990 to September 2013).

- Electronic grey literature databases:

i) Dissertation Abstract (Appendix 12).

ii) Index to Theses (Appendix 13).

We combined the search strategies in PubMed, EMBASE, CINAHL, PsychINFO, ERIC, Ovid, AMED, ASSIA, LILACS, Web of Science, Dissertation Abtracts and Index to Theses with adaptations of the Cochrane RCT search filter as detailed in Lefebvre 2011.

We searched for ongoing clinical trials and unpublished studies via Internet searches on the following websites:
1. www.controlled-trials.com

2. http://clinicalstudyresults.org

3. http://centrewatch.com

\section{Searching other resources}

We searched the reference lists of all relevant papers to identify further studies, as well as conference proceedings likely to contain trials relevant to this Cochrane review. We contacted investigators to ask for information about incomplete trials.

All searches included non-English language literature, and we assessed studies with English language abstracts for inclusion. When considered likely to meet inclusion criteria, the studies were translated to English for subsequent full-text assessment.

\section{Data collection and analysis}

\section{Selection of studies}

One review author (CD) inspected the search hits by reading the titles and the abstracts. We obtained the full text article of each potentially relevant study located in the search and three review authors (CD, BS, JB) independently assessed the article for inclusion. We resolved any doubts about inclusion of a study through discussion, with reference to agreed and written selection criteria.

\section{Data extraction and management}

We used the data collection form template as used by the CDAG. We extracted information from each included study regarding verification of the eligibility of the study in the review, general eligibility criteria specific to this review (including participants, interventions, control group, outcomes), the study characteristics (including methods and specific data relating to participants such as age and sex), details relating to the intervention (such as timing and duration), specific details relating to outcomes measured (including methods of assessment, timings of assessment and length of follow-up), and results for both continuous and dichotomous data for intervention and control arms.

Three review authors (CD, BS, JB) independently extracted data from published sources using a data extraction form. We resolved any disagreements by consensus.

\section{Assessment of risk of bias in included studies}

We assessed risk of bias of the included RCTs by using the criteria recommended in the Cochrane Handbook for Systematic Reviews of Interventions (Higgins 2011). The recommended approach for assessing risk of bias in studies included in a Cochrane review is a two-part tool, addressing eight specific domains, namely: sequence generation and allocation concealment (selection bias), blinding of participants and providers for subjective and objective outcomes 
(performance bias), blinding of outcome assessor for subjective and objective outcomes (detection bias), incomplete outcome data (attrition bias), and selective outcome reporting (reporting bias). The first part of the 'Risk of bias' assessment tool involves describing what was reported to have happened in the study. The second part of the tool involves assigning a judgement relating to the risk of bias for that entry, in terms of 'low', 'high' or 'unclear' risk. To make these judgments we used the criteria indicated by the handbook adapted to the addiction field.

In trials in which no subjective outcomes were utilised (i.e. trial authors used only objective outcomes), we judged performance bias and detection bias for subjective outcomes as unclear risk and stated in the comments section that no subjective outcomes were utilised in the trial. We followed the same process in trials in which no objective outcomes were employed.

We have presented the 'Risk of bias' assessment tool from the CDAG in Appendix 14.

\section{Measures of treatment effect}

In each meta-analysis, data were dichotomous. We analysed dichotomous outcomes by calculating the Risk Ratio (RR) for each trial with the uncertainty in each result being expressed by their confidence intervals (CIs). Continuous data were present in a small number of the single studies which we discussed qualitatively.

\section{Unit of analysis issues}

We included three multi-arm studies in the meta-analysis which were not used more than once in any of the comparisons.

\section{Dealing with missing data}

We contacted the authors of original studies by email (up to three times) for missing data. If no information were available (either from report or the authors) for dichotomous data we assumed that drop-out was due to treatment failure. In cases of missing data about the standard deviation (SD) of the change, we aimed to impute this measure using the SD at the end of treatment for each group. All of the studies analysed in each meta-analysis contained dichotomous data, thus imputing continuous data was not necessary.

\section{Assessment of heterogeneity}

We tested the presence of heterogeneity between the included trials using the $\mathrm{I}^{2}$ statistic. A P value of the $\mathrm{Chi}^{2}$ test $<0.05$ indicated significant heterogeneity.

\section{Assessment of reporting biases}

According to Higgins 2011, tests for funnel plot asymmetry are not viable if all studies are of similar sizes and there are fewer than ten studies in each analysis. We planned to explore the potential for reporting bias further. However, due to the small numbers within each analysis, we did not create funnel plots.

\section{Data synthesis}

We first assessed the effectiveness of psychosocial interventions by considering all types of interventions together (any type) - provided that this made sense from a theoretical, but also practical, approach. We then assessed the effectiveness separately for different types of therapy (i.e. contingency management, psychodynamic approach, counselling). The outcomes from the individual trials were combined through meta-analysis where possible (comparability of intervention and outcomes between trials). We based the choice between random-effects model and fixed-effect model on the observed heterogeneity and on the preliminary assumption about the known or supposed similarity of populations and intervention between the included trials. Fixed-effect meta-analyses ignore heterogeneity, according to Higgins 2011. The populations and interventions evaluated by the studies were so heterogenous that we deemed it more appropriate to use a random-effects model for all analyses.

\section{Subgroup analysis and investigation of heterogeneity}

We had initially planned subgroup analyses for: (i) opiate dependent versus non-opiate dependent; (ii) comparisons between men and women; (iii) residential versus out-patient facility; (iv) harmful use of BZD versus BZD abuse versus BZD dependence; (v) alcohol dependent or not alcohol dependent; (vi) trained people delivering the intervention versus non-trained people; (vii) duration of contact between patient and deliverer of intervention; (viii) supervised withdrawal versus non-specific support; and (ix) gradual or abrupt withdrawal. However, due to the size of the Cochrane review and the complexity of the meta-analyses, we decided to concentrate on the primary outcomes, which would give a set of recommendations that are more clinically relevant and useful. We looked at different follow-up times e.g. post treatment, 3 months, 6 months, 12 months and $>24$ months.

In order to minimise the likelihood of heterogeneity either as a result of methodological diversity (e.g. studies with markedly different durations of follow-up timelines) or clinical diversity (e.g. patient characteristics), we utilised the strategies for addressing heterogeneity outlined in Higgins 2011.

\section{Sensitivity analysis}

To incorporate assessment in the review process we first plotted the intervention effects estimates stratified for risk of bias for each relevant domain. If differences in results were present among studies at different risk of bias, we performed a sensitivity analysis excluding from the analysis studies at high risk of bias. We performed subgroup analysis for studies at low and unclear risk of bias for each of the categories of bias. 
RE S U L T S

\section{Description of studies}

See: Characteristics of included studies; Characteristics of excluded studies.

\section{Results of the search}

We identified 4227 studies (2572 after duplicates removed). Of these, we excluded 2511 on the basis of title and abstract, and retrieved 61 full text articles. Of these 61, we excluded 30 and listed the reason for exclusion in the Characteristics of excluded studies section. We included 25 studies (31 references), of which 15 studies were included in quantitative synthesis (meta-analyses). See Figure 1. 
Figure I. Study flow diagram.

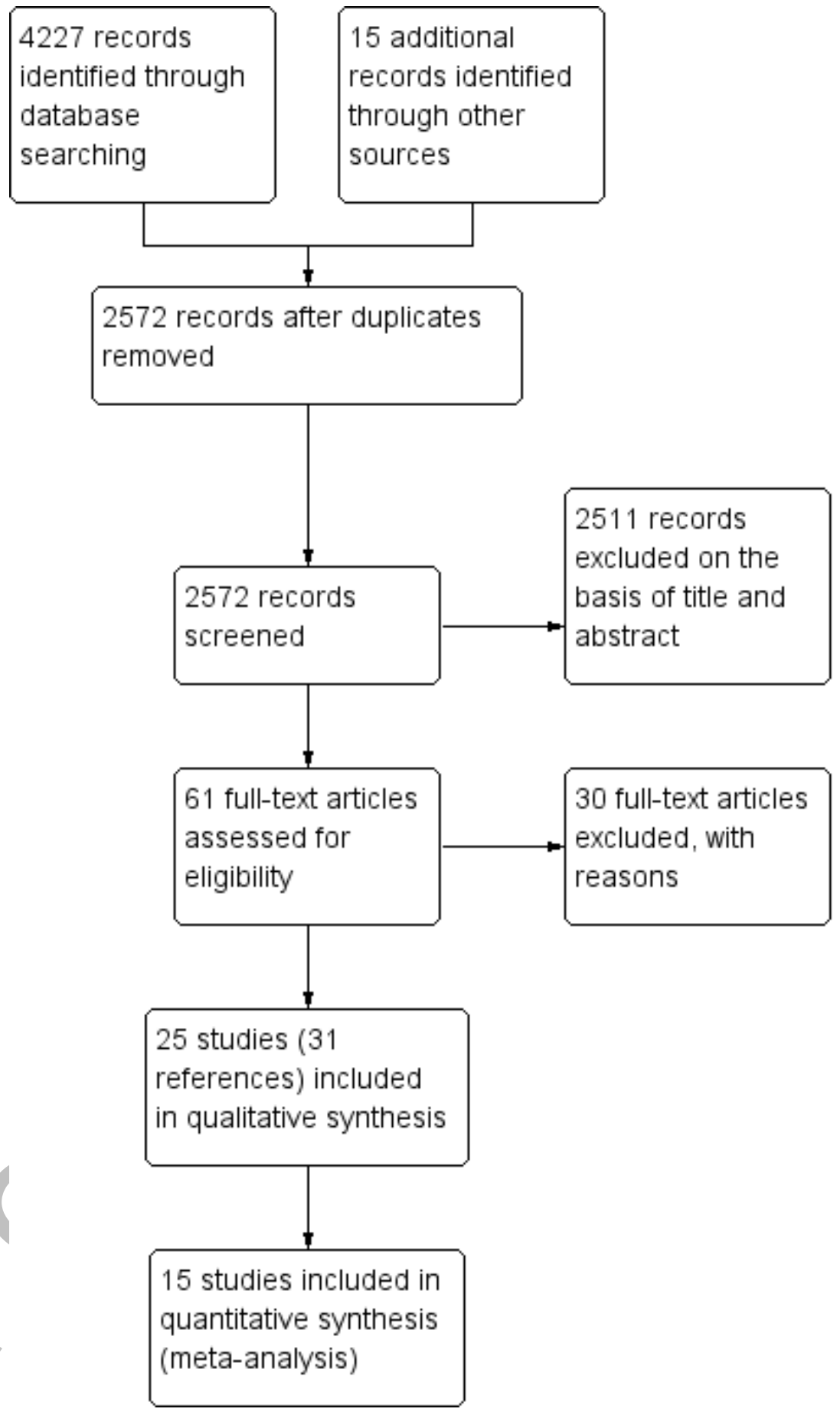




\section{Included studies}

Twenty-five studies (31 references) met the inclusion criteria for this review (see Characteristics of included studies).

\section{Type of psychosocial intervention}

Of the 25 included studies, 11 studies utilised CBT plus taper (Baillargeon 2003; Belleville 2007; Gosselin 2006; Morin 2004; O'Connor 2008; Otto 1993; Otto 2010; Oude Voshaar 2003; Parr 2013; Spiegel 1994; Vorma 2002; ). Two studies utilised CBT without taper (Baker 2005; Scherbaum 2005). Four studies utilised MI (Bagøien 2013; Becka 2004; Carroll 2006; Zahradnik 2009). Two studies utilised letters (Heather 2004; Ten Wolde 2008). Four studies utilised relaxation orientated interventions (Elliott 2005; Elsesser 1996; Gilbert 1993; Nathan 1986). One study used e-counselling (King 2009). One study used advice from a general practitioner (GP) (Vicens 2006).

\section{Participants and settings}

We performed a meta-analysis in relation to CBT comprising 11 unique studies. These studies included a total of 575 participants, 368 women and 207 men. Mean ages in various studies were 55 , $42,39,36$, 'all over 50'. The settings were mostly specialised clinics for insomnia (three trials), panic/anxiety (four trials) and a number of primary care settings where people were on long term BZDs. Six studies were conducted in Canada, three in the USA, one in the Netherlands and one in Finland.

Another four unique studies examining MI were the subject of a separate meta-analysis. There were 80 participants in these studies, 32 women, 38 men and 10 with sex not stated. Two studies took place in opiate dependency clinics (34 participants) and the other two in the acute hospital setting, gynaecology (39 participants) and psychiatry (seven participants). Ages were not given in the opiate clinics. Of the 31 opiate-dependent participants where gender was specified, 23 were men. Studies were conducted in Norway, the Czech Republic, USA and Germany.

The other included studies were not the subject of meta-analysis. Baker 2005 used brief cognitive behavioural interventions for regular amphetamine users. The comparator group were given the same self-help booklet that the intervention group received. The participants were 214 regular amphetamine users recruited through public advertisements in Brisbane, Queensland and Newcastle, New South Wales, Australia.

Elliott 2005 utilised an enhanced intervention consisting of skills training and reinforcement. The comparator was a limited intervention where patients initially received skills training and thereafter only advice. The participants were 119 illicit drug users un- dergoing mandatory reduction of BZD prescription in Dundee, Scotland.

The intervention relating to Elsesser 1996 comprised complaints management training and the comparator comprised anxiety management training. The participants were 44 chronic BZD users recruited through public advertisements and an outpatient treatment centre in Wuppertal, Germany.

Gilbert 1993 reported that there were multiple components to the intervention. These included alerting the patients' doctors, relaxation courses, eight 40-minute sessions over three weeks and the handing out of information. The comparator is not described and is assumed to be none of the above. The participants were 60 residents of aged-care accommodation, who were chronic BZD users in Adelaide, South Australia. The intervention aimed to reduce BZD use.

Heather 2004 reported on the intervention of a letter signed by a GP advising gradual reduction in BZD intake. One comparator was the offer of a short consultation with the patient's GP (or practice nurse/pharmacist) and the other was usual GP care plus assessment. The participants were 299 patients of a range of GPs in Newcastle, England who were long term BZD users.

The intervention relating to King 2009 comprised an internetbased videoconferencing platform for delivering intensified substance abuse counselling. The comparator was onsite group counselling. The participants were 37 illicit drug users attending outpatient drug treatment in Baltimore, Maryland, USA.

Nathan 1986 used supportive withdrawal, weekly 10-minute sessions to stimulate counselling and encouragement of traditional medical care. The comparator was bio-feedback assisted stress management, with individual weekly therapy for 10 weeks. The participants were seven people with BZD dependence recruited through public means in Shreveport, Louisiana, USA.

The intervention reported in Scherbaum 2005 was group psychotherapy, 20 sessions over 20 weeks, and the comparator was treatment as usual (TAU). The participants were 73 opiate addicts attending a methadone maintenance clinic at a psychiatric department of a university hospital in Essen, Germany.

Ten Wolde 2008 reported on use of a computer-generated tailored patient education intervention of varying intensity and the comparator was an existing letter that Dutch GPs use to inform patients about BZD discontinuation. The participations were 508 chronic BZD users recruited through 30 general practices throughout the Netherlands.

Vicens 2006 used standardised advice supplemented with a tapering off schedule with biweekly follow-up visits and the comparator was standardised advice. The participants were 139 adults taking BZDs for more than a year in one of three urban healthcare centres in Mallorca, Spain. 


\section{Excluded studies}

Thirty studies did not meet the criteria for inclusion in this review. We excluded these studies for the following reasons: type of intervention (one study), study design (ten studies), type of participants (two studies), type of participants and type of intervention (one study), type of outcomes (16 studies) (see Characteristics of excluded studies section).

\section{Risk of bias in included studies}

We included 25 trials in this Cochrane review. We have presented the results of our 'Risk of bias' assessment for each included study ( Figure 2) and as percentages across all included studies (Figure 3). We have provided further details of 'Risk of bias' judgements for each included study in the Characteristics of included studies tables. 
Figure 2. Methodological quality summary: review authors' judgements about each methodological quality item for each included study.

\begin{tabular}{|c|c|c|c|c|c|c|c|c|}
\hline & 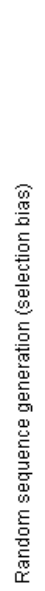 & 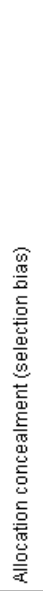 & 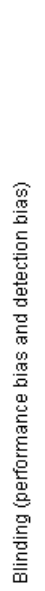 & 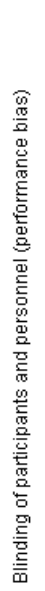 & 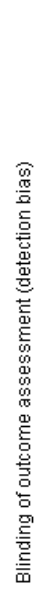 & 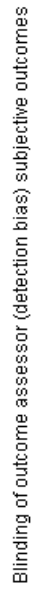 & 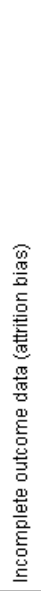 & 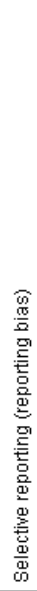 \\
\hline Bagøien 2013 & + & + & $?$ & $\Theta$ & $?$ & $\Theta$ & $\Theta$ & $\odot$ \\
\hline Baillargeon 2003 & 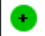 & $?$ & 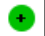 & - & 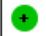 & $\odot$ & 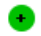 & + \\
\hline Baker 2005 & 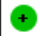 & 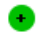 & $?$ & - & $?$ & $\odot$ & $\odot$ & + \\
\hline Becka 2004 & $\odot$ & $\Theta$ & $?$ & $?$ & 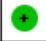 & $?$ & $\odot$ & $\odot$ \\
\hline Belleville 2007 & + & $\Theta$ & ? & $\Theta$ & $?$ & $\odot$ & $\odot$ & + \\
\hline Carroll 2006 & $\oplus$ & $?$ & $?$ & $\odot$ & 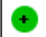 & $?$ & $\odot$ & 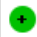 \\
\hline Elliott 2005 & + & + & $\odot$ & $\Theta$ & + & $\odot$ & $\odot$ & + \\
\hline Elsesser 1996 & $?$ & ? & $?$ & $\odot$ & $?$ & $?$ & $\odot$ & $\odot$ \\
\hline Gilbert 1993 & + & $?$ & + & $\Theta$ & + & $?$ & $\odot$ & + \\
\hline Gosselin 2006 & $\oplus$ & 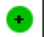 & $\bullet$ & $\odot$ & 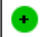 & $\odot$ & $\odot$ & 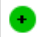 \\
\hline Heather 2004 & $?$ & + & $\Theta$ & ? & + & $?$ & + & + \\
\hline King 2009 & $?$ & ? & $?$ & $\odot$ & 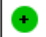 & $?$ & $\odot$ & $\odot$ \\
\hline Morin 2004 & + & + & 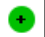 & $\odot$ & 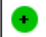 & $\odot$ & $\odot$ & 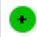 \\
\hline Nathan 1986 & $?$ & ? & $\bullet$ & $?$ & 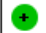 & $?$ & $\odot$ & $?$ \\
\hline O'Connor 2008 & 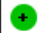 & ? & $\odot$ & $\odot$ & 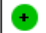 & $?$ & $?$ & + \\
\hline Otto 1993 & 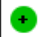 & ? & $?$ & $\odot$ & $?$ & $\odot$ & $\odot$ & $\odot$ \\
\hline Otto 2010 & + & ? & + & $\Rightarrow$ & + & $\odot$ & $\odot$ & + \\
\hline Oude Voshaar 2003 a & + & ? & ? & - & 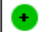 & $?$ & $\odot$ & $\odot$ \\
\hline Parr 2013 & + & + & $?$ & - & $?$ & $\odot$ & $\odot$ & + \\
\hline Scherbaum 2005 & + & ? & + & ? & + & $?$ & 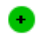 & + \\
\hline Spiegel 1994 & $?$ & ? & ? & $\oplus$ & $?$ & 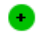 & $\odot$ & + \\
\hline Ten Wolde 2008 & $?$ & - & ? & - & $?$ & $\odot$ & 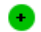 & + \\
\hline Vicens 2006 & 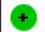 & + & ? & $\odot$ & $?$ & $\odot$ & $\odot$ & $?$ \\
\hline Vorma 2002 & $?$ & + & 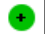 & ? & + & $?$ & $\odot$ & $\odot$ \\
\hline Zahradnik 2009 & $?$ & - & ? & - & $?$ & $\odot$ & $\odot$ & $\odot$ \\
\hline
\end{tabular}

Psychosocial interventions for benzodiazepine harmful use, abuse or dependence (Review)

Copyright $\odot 2015$ The Cochrane Collaboration. Published by John Wiley \& Sons, Ltd. 
Figure 3. Methodological quality graph: review authors' judgements about each methodological quality item presented as percentages across all included studies.

\begin{tabular}{|c|c|}
\hline Random sequence generation (selection bias) \\
Allocation concealment (selection bias) \\
Blinding (performance bias and detection bias) \\
Blinding of participants and personnel (performance bias) \\
Blinding of outcome assessor (detection bias) subjective outcomes \\
Incomplete outcome data (attrition bias) \\
\hline Low risk of bias
\end{tabular}

\section{Allocation}

\section{Random sequence generation}

We considered 16 studies to be at low risk of bias (Bagøien 2013; Baillargeon 2003; Baker 2005; Belleville 2007; Carroll 2006; Elliott 2005; Gilbert 1993; Gosselin 2006; Morin 2004; O’Connor 2008; Otto 1993; Otto 2010; Oude Voshaar 2003 a; Parr 2013; Scherbaum 2005; Vicens 2006). Eight studies (Elsesser 1996; Heather 2004; King 2009; Nathan 1986; Spiegel 1994; Ten Wolde 2008; Vorma 2002; Zahradnik 2009) were judged as at unclear risk of bias and one study as at high risk (Becka 2004).

\section{Allocation concealment}

We judged nine studies to be at low risk of bias (Bagøien 2013; Baker 2005; Elliott 2005; Gosselin 2006; Heather 2004; Morin 2004; Parr 2013; Vicens 2006; Vorma 2002), 12 studies to be at unclear risk of bias (Baillargeon 2003; Carroll 2006; Elsesser 1996; Gilbert 1993; King 2009; Nathan 1986; O’Connor 2008; Otto 1993; Otto 2010; Oude Voshaar 2003 a; Scherbaum 2005; Spiegel 1994) and four studies at high risk of bias (Becka 2004; Belleville 2007; Ten Wolde 2008; Zahradnik 2009).

\section{Blinding}

We considered 10 studies to be at low risk of bias because participants and providers were blinded and it was unlikely that the blinding could have been broken(Baillargeon 2003; Elliott 2005; Gilbert 1993; Gosselin 2006; Morin 2004; Nathan 1986; O'Connor 2008; Otto 2010; Scherbaum 2005; Vorma 2002) . Fourteen studies were judged to be at unclear risk of bias because it was not clear if a blinding condition had been undertaken (Bagøien 2013; Baker 2005; Becka 2004; Belleville 2007; Carroll 2006; Elsesser 1996; King 2009; Otto 1993; Oude Voshaar 2003 a; Parr 2013; Spiegel 1994; Ten Wolde 2008; Vicens 2006; Zahradnik 2009). We judged one study at high risk of bias (Heather 2004).

\section{Blinding of participants and personnel}

Two studies were judged to be at low risk of bias (O'Connor 2008; Spiegel 1994). We judged five studies to be at unclear risk of bias (Becka 2004; Heather 2004; Nathan 1986; Scherbaum 2005; Vorma 2002) and 18 studies at high risk of bias (Bagøien 2013; Baillargeon 2003; Baker 2005; Belleville 2007; Carroll 2006; Elliott 2005; Elsesser 1996; Gilbert 1993; Gosselin 2006; King 2009; Morin 2004; Otto 1993; Otto 2010; Oude Voshaar 2003 a; Parr 2013; Ten Wolde 2008; Vicens 2006; Zahradnik 2009). 
We judged 15 studies to be at low risk of bias(Baillargeon 2003; Becka 2004; Carroll 2006; Elliott 2005; Gilbert 1993; Gosselin 2006; Heather 2004; King 2009; Morin 2004; Nathan 1986; O'Connor 2008; Otto 2010; Oude Voshaar 2003 a; Scherbaum 2005; Vorma 2002). Ten studies were judged to be at unclear risk of bias (Bagøien 2013; Baker 2005; Belleville 2007; Elsesser 1996; Otto 1993; Parr 2013; Spiegel 1994; Ten Wolde 2008; Vicens 2006; Zahradnik 2009) and no studies were judged as high risk.

\section{Blinding of outcome assessor}

We considered six studies at low risk of bias because the trial authors specified that the outcome assessor was blinded (Baker 2005; Elliott 2005; Otto 2010; Parr 2013; Spiegel 1994; Zahradnik 2009). Eleven studies were judged at unclear risk of bias because it was unclear if the outcome assessors were blinded to treatment allocation (Becka 2004; Carroll 2006; Elsesser 1996; Gilbert 1993; Heather 2004; King 2009; Nathan 1986; O’Connor 2008; Oude Voshaar 2003 a; Scherbaum 2005; Vorma 2002). We judged eight studies at high risk of bias because there was no blinding of outcome assessments (Bagøien 2013; Baillargeon 2003; Belleville 2007; Gosselin 2006; Morin 2004; Otto 1993; Ten Wolde 2008; Vicens 2006).

\section{Incomplete outcome data}

We judged that 21 studies were at low risk of attrition bias because all randomised patients were reported/analysed in the group to which they were allocated by randomisation, irrespective of noncompliance and co-interventions (intention-to-treat (ITT)) or had no missing outcome data (Baillargeon 2003; Baker 2005; Becka 2004; Belleville 2007; Carroll 2006; Elliott 2005; Gilbert 1993; Gosselin 2006; Heather 2004; King 2009; Morin 2004; Otto 1993; Otto 2010; Oude Voshaar 2003 a; Parr 2013; Scherbaum 2005; Spiegel 1994; Ten Wolde 2008; Vicens 2006; Vorma 2002; Zahradnik 2009). One study, O'Connor 2008, was judged at unclear risk of bias as the number of drop-outs were not reported for each group. We considered three studies at high risk of attrition bias as there was an imbalance in numbers across groups and 'as treated' analysis was performed (Bagøien 2013; Elsesser 1996; Nathan 1986).

\section{Selective reporting}

Twenty three studies were judged at low risk of reporting bias as study protocols were available (Bagøien 2013; Baillargeon 2003; Baker 2005; Becka 2004; Belleville 2007; Carroll 2006; Elliott 2005; Elsesser 1996; Gilbert 1993; Gosselin 2006; Heather 2004; King 2009; Morin 2004; O'Connor 2008; Otto 1993; Otto 2010; Oude Voshaar 2003 a; Parr 2013; Scherbaum 2005; Spiegel 1994; Ten Wolde 2008; Vorma 2002; Zahradnik 2009). We considered two studies at unclear risk of bias because it was unclear if pre- specified variables had been reported (Nathan 1986; Vicens 2006). No studies were judged at high risk of bias.

\section{Effects of interventions}

See: Summary of findings for the main comparison CBT (plus taper) versus taper for BZD harmful use, abuse or dependence; Summary of findings $2 \mathrm{MI}$ versus TAU for BZD harmful use, abuse or dependence

We could not perform meta-analysis of all included studies. Comparison 1 and comparison 2 provide meta-analytic synthesis. We summarised results according to the type of psychosocial intervention with comparisons of quantitative data where possible. Five studies (seven references) contained three arms and were entered into two separate comparisons (group and single format), so they were not counted twice.

\section{Comparison I: CBT (taper) versus taper}

We counted 11 studies with 575 participants at entry in this comparison. See Summary of findings for the main comparison.

\section{Successful discontinuation of BZDs}

Nine studies reported outcomes within four weeks post-treatment (Baillargeon 2003; Belleville 2007; Gosselin 2006; Morin 2004; O'Connor 2008; Otto 1993; Otto 2010; Spiegel 1994; Vorma 2002) and nine studies reported outcomes at three month followup (Baillargeon 2003; Gosselin 2006; O'Connor 2008; Morin 2004; Otto 1993; Otto 2010; Oude Voshaar 2003 a; Parr 2013; Spiegel 1994). Three studies reported outcomes at six month follow-up (Gosselin 2006; Vorma 2002; Morin 2004). One study reported outcomes at 11 month follow-up (O'Connor 2008). Four studies reported outcomes for 12 month follow-up (Baillargeon 2003; Gosselin 2006; Morin 2004; Vorma 2002) and one study reported outcomes at 15 months follow-up (Oude Voshaar 2003 a). Two studies reported outcomes greater than 24 month followup (Morin 2004; Spiegel 1994).

Trial authors provided additional unpublished data (Belleville 2007; Vorma 2002)

\section{I.I to I.3 Successful discontinuation of BZDs}

We performed meta-analysis on dichotomous data for the number of participants that successfully discontinued BZDs. Results showed a significant difference within four weeks post treatment (RR 1.40, 95\% CI 1.05 to 1.86; nine studies, 423 participants) and at three month follow-up (RR $1.51,95 \%$ CI 1.15 to 1.98 ; 9 studies, 460 participants) in favour of CBT (taper) for the successful discontinuation of BZDs. However, there was significant heterogeneity at post treatment $\left(\mathrm{I}^{2}\right.$ statistic $\left.=60 \%, \mathrm{P}=0.01\right)$ and three month follow-up $\left(\mathrm{I}^{2}\right.$ statistic $\left.=40 \%, \mathrm{P}=0.10\right)$. Few studies 
contributed to the meta-analysis for subsequent follow-up assessments, thus no significant difference between CBT (taper) and taper for the successful discontinuation of BZDs was found at six month (RR 1.94, 95\% CI 0.88 to 4.30; three studies, 155 participants), $11 / 12$ month (RR $1.42,95 \%$ CI 0.89 to 2.28 ; five studies, 284 participants), 15 month (RR $0.80,95 \%$ CI 0.49 to 1.31 ; one study, 146 participants) and greater than 24 month followup (RR 1.77, 95\% CI 0.98 to 3.17; two studies, 73 participants). See Analysis 1.1.

We performed a sensitivity analysis excluding studies at high risk of bias for allocation concealment. Results indicate significant difference within four weeks post treatment follow-up (RR 1.50, 95\% CI 1.12 to 2.02; eight studies, 370 participants) in favour of CBT (taper). Heterogeneity remained significant $\left(\mathrm{I}^{2}\right.$ statistic $=55 \%, \mathrm{P}$ $=0.03$ ). See Analysis 1.2.

We conducted a sensitivity analysis excluding studies at high risk of bias for blinding of outcome assessor. Results indicated no significant difference within four weeks post treatment follow-up (RR 1.08, 95\% CI 0.73 to 1.59; four studies, 159 participants). However, significant difference was found at three month followup (RR 1.60, 95\% CI 1.08 to 2.36; four studies, 103 participants) and greater than 24-month follow-up in favour of CBT (taper) (RR 2.73, 95\% CI 1.02 to 7.32; one study, 21 participants). No significant difference was found at six month follow-up (RR 1.98, 95\% CI 0.37 to 10.47 ; two studies, 94 participants); 12 month follow-up (RR 0.70, 95\% CI 0.28 to 1.79 ; one study, 62 participants). Heterogeneity was not significant post treatment ( $\mathrm{I}^{2}$ statistic $=44 \%, P=0.15)$ and at three-month follow-up ( $I^{2}$ statistic $=$ $0 \%, \mathrm{P}=0.55)$. However it was significant at six month follow-up $\left(\mathrm{I}^{2}\right.$ statistic $\left.=78 \%, \mathrm{P}=0.03\right)$. See Analysis 1.3 .

\section{I.4 Reduce BZD by $>50 \%$}

Three studies reported outcomes within four weeks post-treatment (Baillargeon 2003; Belleville 2007; Vorma 2002), two studies reported outcomes at three months follow-up (Baillargeon 2003; Parr 2013) and one study at six months follow-up (Vorma 2002). Two studies reported outcomes at 12 months follow-up (Baillargeon 2003; Vorma 2002).

We received additional unpublished data from the trial authors of Vorma 2002.

Few studies contributed to the meta-analysis on dichotomous data for the number of participants that successfully reduced BZDs greater than $50 \%$. There was no significant difference between CBT (taper) and TAU at any time point - within four weeks post treatment (RR 0.93, 95\% CI 0.11 to 8.18; three studies, 178 participants), three month follow-up (RR $1.99,95 \%$ CI 0.47 to 8.47; two studies, 69 participants), six month (RR 0.76, 95\% CI 0.28 to 2.07; one study, 62 participants) and 12 month follow-up (RR 1.07, 95\% CI 0.14 to 8.21; two studies, 125 participants). See Analysis 1.4 .

\section{I.5 Drop-outs/lost to follow-up}

Nine studies reported post-treatment drop-outs (Baillargeon 2003; Belleville 2007; Gosselin 2006; Morin 2004; O’Connor 2008; Otto 1993; Otto 2010; Oude Voshaar 2003 a; Spiegel 1994), one study reported drop-outs/participants lost to followup outcomes by three month follow-up (Baillargeon 2003; 65 participants), one study reported drop-out/lost to follow-up by six month follow-up (Vorma 2002) and two studies reported dropouts/lost to follow-up by 12 month follow-up (Baillargeon 2003; Vorma 2002).

There was no significant difference at any of the time intervals; within four weeks post-treatment (RR 1.05, 95\% CI 0.66 to 1.66 ; nine studies, 478 participants), three months follow-up (RR 1.71, $95 \%$ CI 0.16 to 17.98 ; one study, 65 participants), six months follow-up (RR 0.70, 95\% CI 0.17 to 2.88 ; one study, 62 participants) and 12 month follow-up (RR 2.57, 95\% CI 0.28 to 23.44; one study, 65 participants). See Analysis 1.5.

\section{Comparison 2: MI versus TAU}

We included four studies with 80 participants at entry in this comparison. See Summary of findings 2.

We received additional unpublished data from the trial authors of Bagøien 2013, Becka 2004 and Zahradnik 2009.

Two studies reported outcomes within four weeks post treatment (Becka 2004; Carroll 2006), four studies reported outcomes at three month follow-up (Bagøien 2013; Becka 2004; Carroll 2006; Zahradnik 2009), one study reported outcomes at six month follow-up (Bagøien 2013) and two studies reported outcomes at 12 month follow-up (Bagøien 2013; Zahradnik 2009).

\section{I Successful discontinuation of BZDs}

Meta-analysis on dichotomous data for the number of participants that successfully discontinued BZDs indicated no statistically significant difference at any of the time intervals; post treatment (RR 4.43, $95 \%$ CI 0.16 to 125.35 ; two studies, 34 participants), three months follow-up (RR 3.46, 95\% CI 0.53 to 22.45 ; four studies, 80 participants). See Analysis 2.1 .

\subsection{Reduce BZD by $>50 \%$}

Meta-analysis on dichotomous data for the number of participants that successfully reduced BZDs greater than 50\% indicated insufficient evidence to suggest a statistically significant difference at any of the time intervals; three months follow-up (RR 1.52, 95\% CI 0.60 to 3.83; one study, 39 participants) and 12 months followup (RR 0.87, 95\% CI 0.52 to 1.47; one study, 39 participants). See Analysis 2.2. 


\section{Comparison 3: GP advice (taper) versus TAU}

We included one study with 139 participants in this comparison. The trial authors of Vicens 2006 provided unpublished data.

\section{I Successful discontinuation of BZDs}

Analysis of dichotomous data for the number of participants who successfully discontinued BZDs indicated a significant difference at six months follow-up (RR 13.11, 95\% CI 3.25 to 52.83) and 12 months follow-up (RR 4.97, 95\% CI 2.23 to 11.11) in favour of standardised interview. No statistically significant difference between treatments was found at three years follow-up (RR 1.61, 95\% CI 0.92 to 2.84). See Analysis 3.1.

\subsection{Reduce BZD by $>\mathbf{5 0} \%$}

Analysis of dichotomous data for the number of participants who successfully reduced BZDs by $50 \%$ indicated a significant difference at six months follow-up (RR 3.32, 95\% CI 1.43 to 7.67 ) and 12 months follow-up (RR 13.11, 95\% CI 3.25 to 52.83 ) in favour of standardised interview. See Analysis 3.2.

\section{Comparison 4: CBT (no taper) versus TAU}

We included one study of 73 participants in this comparison ( Scherbaum 2005).

\section{I BZD positive urine rate}

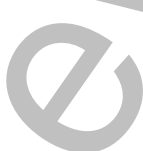

Analysis of continuous data indicated no statistically significant difference post treatment (mean difference (MD) - $0.01,95 \% \mathrm{CI}$ 0.19 to 0.17 ), three months follow-up (MD - 0.08 , 95\% CI -0.25 to 0.09 ) and six months follow-up (MD $-0.09,95 \% \mathrm{CI}-0.25$ to 0.07). See Analysis 4.1.

\section{Comparison 5: Self-help booklet plus CBT versus self- help booklet}

We included one study of 29 participants in this comparison ( Baker 2005).

We obtained unpublished data from the trial author.

\section{I Change in OTI score for BZD use}

Analysis of continuous data indicated no statistically significant difference post treatment (MD 0.73, 95\% CI -1.94 to 3.40) and six months follow-up (MD $-0.27,95 \%$ CI -4.06 to 3.52 ). See Analysis 5.1.

\section{Comparison 6: Complaints management (additional relaxation) versus anxiety management (relaxation)}

For this comparison we included one study of 19 participants (Elsesser 1996).

\section{I Successful discontinuation of BZDs}

Analysis of dichotomous data for the number of participants who successfully discontinued BZDs indicated there was no difference post treatment (RR 1.56, $95 \% \mathrm{CI} 0.76$ to 3.17) and at six months follow-up (RR 0.93, 95\% CI 0.43 to 2.01). See Analysis 6.1.

\section{Comparison 7: Consultation (plus letter) versus TAU}

We included one study with 272 participants in this comparison (Heather 2004).

\section{I Successful discontinuation of BZDs}

Analysis of dichotomous data for the number of participants who successfully discontinued BZDs indicated there was no significant difference at six months follow-up (RR 1.54, 95\% CI 0.64 to 3.72). See Analysis 7.1.

\section{Comparison 8: E-counselling versus onsite counselling}

For this comparison, we included one study of 37 participants (King 2009).

We obtained unpublished data from the trial author.

\section{I Positive BZD urine toxicology}

Analysis of continuous data indicated no statistically significant difference six weeks follow-up (MD -0.01, 95\% CI -0.04 to 0.02; Analysis 8.1).

\section{Comparison 9: Relaxation versus TAU}

We included one study with 60 participants in this comparison (Gilbert 1993).

\section{I Successful discontinuation of BZDs}

Analysis of dichotomous data for the number of participants who successfully discontinued BZDs indicated no statistically significant difference post treatment (RR $1.90,95 \%$ CI 0.98 to 3.70 ) but there was a statistically significant difference at three months follow-up (RR 2.20, 95\% CI 1.23 to 3.94) in favour of relaxation. See Analysis 9.1. 


\section{Comparison 10: Tailored letter versus GP letter}

For this comparison we included one study with 322 participants (Ten Wolde 2008).

\section{I Successful discontinuation of BZDs}

Analysis of dichotomous data for the number of participants who successfully discontinued BZDs indicated a statistically significant difference at 12 months follow-up in favour of tailored letter (RR $1.70,95 \%$ CI 1.07 to 2.70; Analysis 10.1).

\section{Comparison I I: Taper (relaxation) versus taper only}

One study of 31 participants was included in this comparison (Otto 2010).

\section{I.I Successful discontinuation of BZDs}

Analysis of dichotomous data for the number of participants who successfully discontinued BZDs indicated no statistically significant difference post treatment (RR $0.78,95 \%$ CI 0.30 to 2.03), at three months follow-up (RR $0.47,95 \%$ CI 0.10 to 2.20 ) or six months follow-up (RR $0.47,95 \%$ CI 0.10 to 2.20 ) in favour of tailored letter. See Analysis 11.1.

\section{Comparison I2: Enhanced skills training (relaxation) versus limited skills training (relaxation)}

We included one study with 53 participants in this comparison (Elliott 2005).

\section{I Change in prescribed diazepam dose (mg)}

Analysis of continuous data indicated no statistically significant difference at six months follow-up (MD 4.40, 95\% CI -0.01 to 8.81; Analysis 12.1).

We took a very broad approach, both in terms of defining an intervention and picking a condition/diagnosis to examine. Due to these factors we already had a degree of heterogeneity and analysis of secondary outcomes would have given rise to a less accessible and intelligible review. Concentrating on the primary outcome gives a set of messages that are more clinically relevant and useful. Several authors with clinical backgrounds read all included studies. One of the notable features was the absence of commentary on adverse effects in the papers. 


\begin{tabular}{|c|c|c|c|c|c|}
\hline \multicolumn{6}{|c|}{ MI versus TAU for BZD harmful use, abuse or dependence } \\
\hline \multicolumn{6}{|c|}{$\begin{array}{l}\text { Patient or population: patients with BZD harmful use, abuse or dependence } \\
\text { Settings: outpatient } \\
\text { Intervention: MI versus TAU }\end{array}$} \\
\hline \multirow[t]{3}{*}{ Outcomes } & \multicolumn{2}{|c|}{ Illustrative comparative risks* $(95 \% \mathrm{Cl})$} & \multirow{3}{*}{$\begin{array}{l}\text { Relative effect } \\
(95 \% \mathrm{Cl})\end{array}$} & \multirow{3}{*}{$\begin{array}{l}\text { No of participants } \\
\text { (studies) }\end{array}$} & \multirow{3}{*}{$\begin{array}{l}\text { Quality of the evidenc } \\
\text { (GRADE) }\end{array}$} \\
\hline & Assumed risk & Corresponding risk & & & \\
\hline & Control & MI versus TAU & & & \\
\hline \multirow{4}{*}{$\begin{array}{l}\text { Successful discontinuation } \\
\text { of BZDs- post treatment } \\
\text { Objective and subjective } \\
\text { Follow-up: mean } 6 \text { weeks }\end{array}$} & Study populatic & & \multirow{4}{*}{$\begin{array}{l}\text { RR } 4.43 \\
-(0.16 \text { to } 125.35)\end{array}$} & \multirow{4}{*}{$\begin{array}{l}34 \\
\text { (2 studies) }\end{array}$} & \multirow{4}{*}{$\begin{array}{l}\oplus \bigcirc \bigcirc \bigcirc \\
\text { very low } 1,2\end{array}$} \\
\hline & 59 per 1000 & $\begin{array}{l}261 \text { per } 1000 \\
(9 \text { to } 1000)\end{array}$ & & & \\
\hline & \multicolumn{2}{|l|}{ Moderate } & & & \\
\hline & 250 per 1000 & $\begin{array}{l}1000 \text { per } 1000 \\
(40 \text { to } 1000)\end{array}$ & & & \\
\hline \multirow{4}{*}{$\begin{array}{l}\text { Successful discontinuation } \\
\text { of BZDs- } 3 \text { month follow-up } \\
\text { Objective and subjective } \\
\text { Follow-up: mean } 3 \text { months }\end{array}$} & Study populatic & & \multirow{4}{*}{$\begin{array}{l}\text { RR } 3.46 \\
\text { (0.53 to 22.45) }\end{array}$} & \multirow{4}{*}{$\begin{array}{l}80 \\
\text { (4 studies) }\end{array}$} & \multirow{4}{*}{$\begin{array}{l}\oplus \bigcirc \bigcirc \bigcirc \\
\text { very low } 3,4\end{array}$} \\
\hline & 100 per 1000 & $\begin{array}{l}346 \text { per } 1000 \\
\text { (53 to } 1000)\end{array}$ & & & \\
\hline & \multicolumn{2}{|l|}{ Moderate } & & & \\
\hline & 250 per 1000 & $\begin{array}{l}\mathbf{8 6 5} \text { per } \mathbf{1 0 0 0} \\
(132 \text { to } 1000)\end{array}$ & & & \\
\hline
\end{tabular}

*The basis for the assumed risk (e.g. the median control group risk across studies) is provided in footnotes. The corresponding risk (and its $95 \% \mathrm{Cl}$ ) is based on the assumed risk in the comparison group and the relative effect of the intervention (and its $95 \% \mathrm{Cl}$ ).

Cl: confidence interval; RR: risk ratio; BZD: benzodiazepine; MI: motivational interviewing; TAU: treatment as usual. 


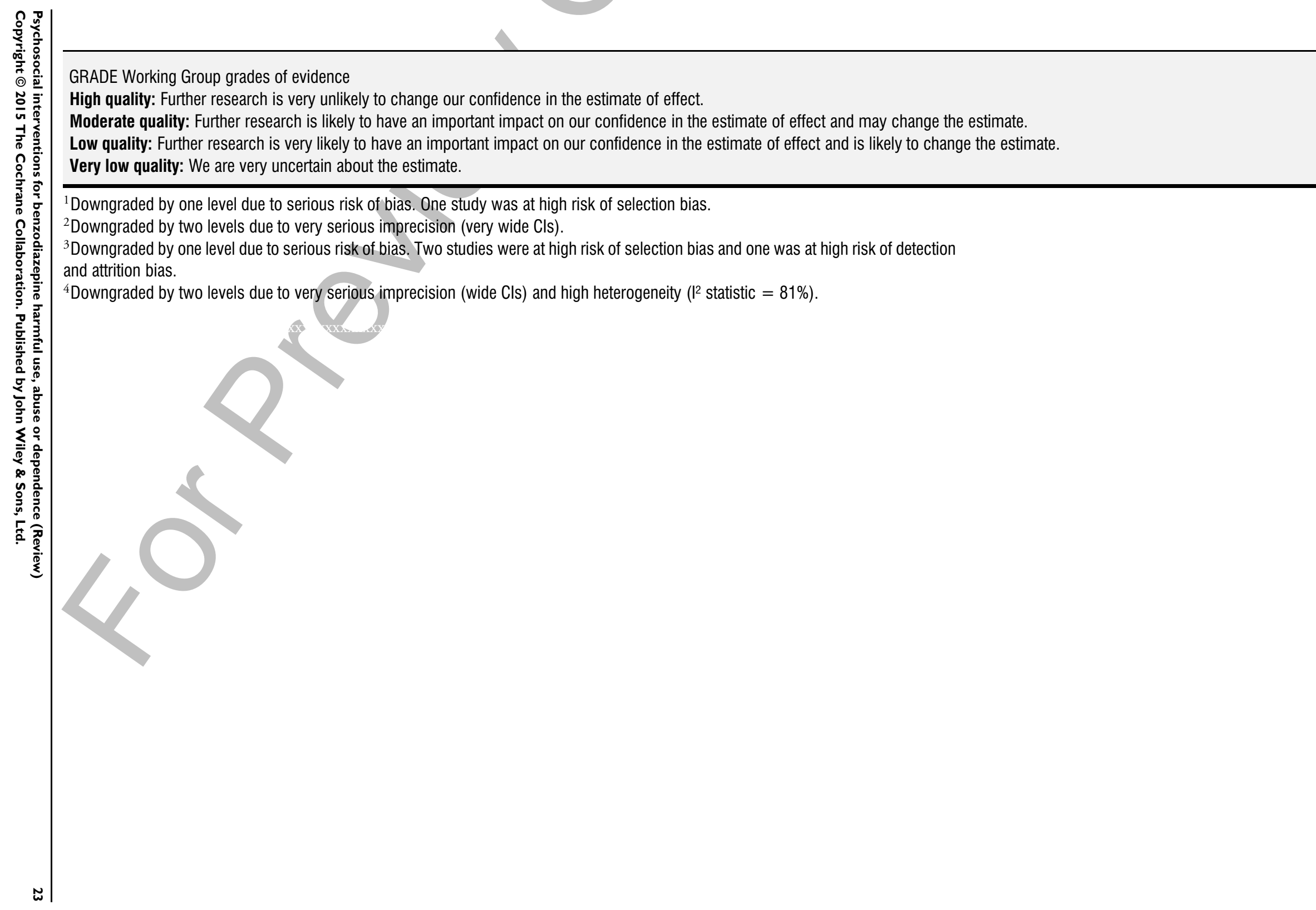




\section{DISCUSSION}

\section{Summary of main results}

The included studies tested an array of different psychosocial interventions, including CBT (some studies with taper, other studies with no taper), MI, letters to patients advising them to reduce or quit BZD use, relaxation studies, counselling delivered electronically and advice provided by a GP.

There was moderate quality of evidence when comparing CBT plus taper versus taper only in the short term (Summary of findings for the main comparison). Comparing CBT plus taper versus taper only, studies showed a statistically significant difference between the treatments in terms of successful discontinuation of BZDs within four weeks post treatment (RR 1.40, 95\% CI 1.05 to 1.86 ) and at three months follow-up (RR 1.51, 95\% CI 1.15 to 1.98) in favour of CBT (taper) for 575 participants. No significant difference was found at six months, 11/12 months, 15 months and 24 months follow-up. There was moderate quality of evidence at 12 months follow-up (Summary of findings for the main comparison). When assessing the reduction of BZDs by $>50 \%$ results, there was low quality of evidence which showed there was no statistically significant difference at any time point in favour of CBT (taper) (Summary of findings for the main comparison). There was insufficient evidence to determine drop-outs at any of the time intervals; post-treatment (RR $1.05,95 \%$ CI 0.66 to 1.66), three months follow-up (RR $1.71,95 \%$ CI 0.16 to 17.98 ) and six months follow-up (RR 0.70, 95\% CI 0.17 to 2.88).

There was very low quality of data for MI versus TAU at all time points (Summary of findings 2). Comparing MI versus TAU in the 80 participants showed that there was no statistically significant difference between treatments at any of the time intervals; post treatment (RR 4.43, 95\% CI 0.16 to 125.35 ) and at three months follow-up (RR 3.46, 95\% CI 0.53 to 22.45). When assessing the reduction of BZDs by $>50 \%$, results showed insufficient evidence to suggest a statistically significant difference at any of the time intervals (three months follow-up (RR $1.52,95 \%$ CI 0.60 to 3.83 ) and 12 months follow-up (RR $0.87,95 \%$ CI 0.52 to 1.47 ). There was insufficient evidence to suggest a significant difference relating to drop-outs from treatment at any of the time intervals; posttreatment (RR 0.50, 95\% CI 0.04 to 7.10 ), three months followup (RR $0.46,95 \%$ CI 0.06 to 3.28), six months follow-up (RR $8.75,95 \% \mathrm{CI} 0.61$ to 124.53 ) and 12 months follow-up (RR $0.42,95 \%$ CI 0.02 to 7.71 ).

The following single studies significantly reduced BZD use: tailored GP letter versus generic GP letter (Ten Wolde 2008) at 12 months follow-up (RR 1.70, 95\% CI 1.07 to 2.70), standardised interview versus TAU (Vicens 2006) at six months follow-up (RR $13.11,95 \%$ CI 3.25 to 52.83 ) and 12 months follow-up (RR 4.97, 95\% CI 2.23 to 11.11); and relaxation versus TAU (Gilbert 1993) at three months follow-up (RR 2.20, 95\% CI 1.23 to 3.94).
There was insufficient supporting evidence for the remaining single studies.

Furthermore, we adopted adopted a very broad approach in this Cochrane review, and the analysis of secondary outcomes would have given rise to a less accessible and intelligible systematic review. Concentrating on the primary outcome gives a set of messages that are more clinically relevant and useful.

\section{Overall completeness and applicability of evidence}

The objective of this Cochrane review was to measure the effect of a psychosocial intervention on influencing the use of BZDs in people who harmfully use, abuse or are dependent on these substances. Two types of psychosocial interventions (CBT plus taper versus taper only; MI versus TAU)provided enough studies to warrant a meta-analysis. The combined sample size of the CBT plus taper studies was a modest 575 participants, thus limiting the generalisability of the findings. Likewise the four MI studies included 80 participants, which also limits the generalisability of the findings. The other studies of psychosocial interventions including letters and relaxation did not warrant a meta-analysis so there is no synthesised evidence that can be drawn from these types of psychosocial interventions.

Many of the included studies were from non-opiate dependent populations. It is known that methadone-maintained opiate dependent patients may take a BZD after taking methadone to augment the high they experience by increasing sedation. Further high quality trials are needed targeting BZDs in opiate dependent methadone-maintained populations to determine the effectiveness of psychosocial interventions to reduce BZD use in this vulnerable group.

Some of the included studies were not necessarily directly targeting BZD as the target substance, but rather another illicit substance such as amphetamines. It is therefore difficult to interpret from such studies when compared with studies that specifically targeted BZDs.

There was some heterogeneity between the studies relating to the length of treatment; e.g. Vorma 2002, which was a CBT plus taper intervention, had a treatment period of a year.

Many studies include $50 \%$ reduction as a clinically meaningful outcome for participants. This target is included by many clinicians because it can be so difficult for many patients who have been using BZDs for many years to stop.

\section{Quality of the evidence}

Twenty-five studies met the inclusion criteria of this Cochrane review. These are reported across 31 references; six references related to follow-up data. We performed a 'Risk of bias' assessment for all included studies. In general the quality of the evidence was 
low (Summary of findings for the main comparison; Summary of findings 2). The studies tended to have low participant numbers, few studies had manualised sessions or independent tests of treatment fidelity, and most follow-up periods were $<12$ months. As a result, the conclusions of this review should be considered tentative at best. Nonetheless, this review provides an overview of the current status of evidence and points to future directions for research on the capacity of psychosocial interventions to reduce harmful use, abuse and dependence of BZDs. We chose the outcomes considered in the 'Summary of findings' tables after considering the end of treatment and longest available follow-up with relevant number of studies and participants, the most relevant for adding useful information.

\section{Potential biases in the review process}

Limitations of this Cochrane review should be noted. The terms of reference of the review were very wide. Based on decisions made during the implementation of protocol methods to present a manageable summary of the evidence, we did not collect data on quality of life, self-harm or adverse events which were noted at the protocol stage. The definition of psychosocial intervention is by definition broad and this presents a challenge for meta-analysis. In addition, as the included studies spanned almost 30 years, the clinical practice and the description of clinical practice has changed a lot. Some interventions have been very precisely defined and described (i.e. relaxation training and sleep hygiene, with detailed description of exactly what the study participants were exposed to) and others were loosely defined with a single word (i.e. counselling). Another challenge we faced was that for studies conducted preinternetit was more difficult to contact trial authors. We could not use many results in the absence of clarification and more precise ascertainment, thus limiting the power of some meta-analyses and preventing meta-analyses of other psychosocial interventions. Another issue is that there was wide variation in how outcomes were categorised and reported. Studies from 20 and 30 years ago were more discursive, with less reliance on objective outcomes. Also, in some studies the outcome of interest, change in BZD use, was mixed in with changes in use of other substances. Thus we had to discard relevant data.

In all scenarios above we erred on the side of excluding data if we were unsure whether it fell within the scope of this Cochrane review.

\section{Agreements and disagreements with other studies or reviews}

There have been four non-Cochrane meta-analyses (Gould 2014a; Mugunthan 2011; Parr 2008; Oude Voshaar 2006c) and two reviews (Noyes 1988; Smith 2010) on the area of psychosocial interventions to reduce BZD use.
Parr 2008 focused on general practice and out-patient treatment settings and examined both pharmacological and psychosocial treatments for reducing BZD use. Pharamcological treatments alone are outside of the remit of this Cochrane review. Thirty-two studies met the inclusion criteria, 16 of which had a psychosocial component. The trial authors categorised three different types of psychosocial intervention: brief intervention versus routine care; psychological interventions versus routine care; and gradual dose reduction (GDR) plus psychological interventions. Brief intervention, such as a GP sending a letter was found to be more effective than routine care, or not raising the issue at all $(\mathrm{OR}=4.37$, 95\% CI 2.28 to 8.40 ). Psychological interventions, such as relaxation training, psycho education for BZD withdrawal or teaching strategies to reduce insomnia versus routine care, resulted in higher BZD cessation rates than routine care (OR 3.37; 95\% CI 1.86 to 6.12). GDR plus psychological interventions, such as relaxation training, CBT of insomnia, self-monitoring of consumption, goal setting, management of withdrawal and coping with anxiety, were considered slightly more effective than GDR alone at post cessation (OR 1.82, 95\% CI 1.25 to 2.67). Parr 2008 did not disentangle a specific type of psychosocial intervention, such as CBT or $\mathrm{MI}$, as in the current review. However, our findings are congruent with Parr 2008 regarding the additional benefit of CBT plus taper and CBT versus routine care. In our review, a tailored GP letter (Ten Wolde 2008) was more effective than a generic GP letter in reducing BZD use in a single study. However, there was insufficient evidence to suggest that another study which examined a GP consultation plus letter versus TAU was effective (Heather 2004). Oude Voshaar 2006c focused on psychosocial and pharmacological treatments. Pharamcological treatments alone are outside the remit of this Cochrane review. Twenty-nine studies met the inclusion criteria, nine of which were psychosocial treatment studies. Psychosocial treatments were categorised into two broad categories - minimal interventions and systematic discontinuation. Minimal intervention comprised such as simple advice in letter format or meeting to a large group of people and systematic discontinuation comprised such as treatment programmes led by a physician or psychologist. Both types of interventions were found to be significantly more effective than TAU: minimal interventions (pooled OR 2.8, 95\% CI 1.6 to 5.1); systematic discontinuation alone (one study, OR 6.1, 95\% CI 2.0 to 18.6). Systematic discontinuation plus pharmacological treatment (OR 3.1, 95\% CI 1.1 to 9.4) or group CBT for patients with insomnia (OR 5.5, 95\% CI 2.3 to 14.2 ) was superior to systematic discontinuation alone. The evidence from Oude Voshaar 2006c relating to the benefit of CBT plus taper is congruent with this Cochrane review.

Mugunthan 2011 focused on the evidence from primary care studies. Three studies met the inclusion criteria. Psychosocial interventions were minimal interventions, such as a simple tailored letter or a single consultation. The pooled risk ratio showed a significant reduction/cessation in BZD consumption in the minimal intervention groups compared to usual care (RR 2.1, 95\% CI 1.5 to 
2.9; RR 2.4, 95\% CI 1.3 to 4.3). In our review, a tailored GP letter (Ten Wolde 2008) was found to be more effective than a generic GP letter in reducing BZD use in a single study. However, there was insufficient evidence to suggest that another study which examined a GP consultation plus letter versus TAU was effective (Heather 2004).

Gould 2014a focused on the evidence for trials of BZD withdrawal and prescribing interventions in older people aged $\geq 50$ years. Ten withdrawal and eight prescribing studies met the inclusion criteria. For the purposes of comparison with this review, only four trials combining withdrawal with psychotherapy were relevant. Psychotherapy was described as CBT in two studies, relaxation training in one study and psychological consulting in one study. At post-intervention, significantly higher odds of not using BZDs were found with supervised withdrawal with psychotherapy (OR 5.06, 95\% CI 2.68 to $9.57, \mathrm{P}<0.00001$ ) in comparison with the control interventions TAU, education placebo, withdrawal with or without drug placebo, or psychotherapy alone. Gould 2014b submitted a correction to the original meta-analysis indicating that errors had been made in some of the data analyses. All data were re-analysed and the authors concluded that "the patterns of results and conclusions remain unchanged from those originally reported in the review, with the minor exception of the following", which related to the psychotherapeutic analyses: " 1 . There is no longer any evidence of heterogeneity in effect sizes for withdrawal with psychotherapy at 0.5-3 months. Thus, the conclusion that this type of intervention may not always be effective (v. control conditions) in individual settings no longer stands". Consequently, the evidence from Gould 2014a relating to the short term benefit of psychotherapy plus withdrawal is congruent with our review. Smith 2010 and Noyes 1988 were two non-systematic reviews of the evidence for reducing BZD use. Smith 2010 found that studies that used a multi-faceted approach had the largest and most sustained reductions in BZD use. Noyes 1988 focused on the side effects of withdrawal from BZD and found that rebound anxiety occurred in a substantial minority of patients after several weeks; withdrawal syndrome developed in nearly half of patients who used BZD for more than a year.

\section{AUTHORS, CONCLUSIONS Implications for practice}

There is evidence to support the use of CBT plus taper to reduce BZD use in the short term. There is currently no evidence to support the use of MI. In addition, there is some emerging evidence that simple interventions, such as structured consultation and individually tailored GP letters, may be worth exploring further.

\section{Implications for research}

As problem BZD use is a serious global public health issue the need for more focused systematic reviews and for a much more standardised approach to the development, implementation and documentation of psychosocial interventions to assist discontinuation is pressing. The evidence of reductions of BZDs in the GP letter trials (Ten Wolde 2008; Vicens 2006) warrants further research.

Due to the very broad terms of reference of this Cochrane review, we have a number of suggestions emanating from our work:

\section{Populations to study}

The reality of substance use is that polydrug use is the norm. Prescription medications and BZDs in particular are ubiquitous. There are at least three sub-populations of BZD users; those who take them with opiates, those who are prescribed BZDs for sleep disorders and other defined conditions and those who take BZDs (prescribed or street acquired) in a 'recreational' manner. It would be more legitimate for these three sub-populations to be studied separately.

\section{Nature of intervention}

A classification system for psychosocial interventions is overdue. One of the limitations in this review is non-standardisation of definition and description of the intervention. This could be addressed at a European level through the European Monitoring Centre for Drugs and Drug Addiction (EMCDDA) or globally through the WHO.

The evidence for loss of effect between the six and nine month period would benefit from further consideration of some form of modified relapse prevention or booster type of intervention to determine if it would impact on the loss of effect.

Separately, since the GP letter studies have shown some positive effect, the possibility of using technologies such as text-based and internet-based interventions need to be trialed and evaluated.

\section{Outcome measurement}

Guidance on outcome measurement could be given by the addiction literature or by agreement with the editors of relevant journals. This would help greatly to improve the environment in which meta-analyses are performed in the addiction and substance misuse field. Objective outcomes, such as urinanalysis, should be used where possible.

\section{Type of study}

Similar to the preceding point, adherence to guidelines on conducting RCTs would enhance the science and practice of metaanalysis and Cochrane reviews. 


\section{ACKNOW LEDGEMENTS}

We thank Dr Laura Amato (Coordinating Editor, CDAG), Ms Zuzana Mitrova (Managing Editor and Trial search Coordinator, CDAG) and Silvia Minozzi (Quality Advisor and Assistant Managing Editor, CDAG) and Professor Phil Wiffen (Director of Operations and Training at the UK Cochrane Centre) for all of their helpful suggestions and advice, support and patience in writing this protocol and review. We also acknowledge the funding and training provided for the lead review author (CD) by means of a Cochrane Fellowship from the Health Research Board in Ireland, to undertake this review.

\section{R E F E R E N C ES}

\section{References to studies included in this review}

Bagøien 2013 \{published and unpublished data\}

Bagøien G, Bjørngaard J, Østensen C, Reitan S, Romundstad P, Morken G. The effects of motivational interviewing on patients with comorbid substance use admitted to a psychiatric emergency unit - a randomised controlled trial with two year follow-up. BMC Psychiatry 2013;13(93):1-10.

Baillargeon 2003 \{published data only\}

Baillargeon L, Landreville P, Verreault R, Beauchemin J-P, Grégoire J-P, Morin CM. Discontinuation of benzodiazepines among older insomniac adults treated with cognitive-behavioural therapy combined with gradual tapering: a randomized trial. Canadian Medical Association Journal 2003;169(10):1015-20.

Baker 2005 \{published data only\} Baker A, Lee NK, Claire M, Lewin TJ, Grant T, Pohlman $S$, et al. Brief cognitive behavioural interventions for regular amphetamine users: A step in the right direction. Addiction 2005;100(3):367-78.

Becka 2004 \{published data only\} Becka J. Efficacy of cognitive behavioral methods in decreasing drug use by methadone clinic patients compared

with standard conditions. Č eská a Slovenská Psychiatrie 2004;100(Suppl 1):4-7.

Belleville 2007 \{published data only\} Belleville G, Guay C, Guay B, Morin CM. Hypnotic taper with or without self-help treatment of insomnia: a randomized clinical trial. Journal of Consulting and Clinical Psychology 2007;75(2):325-35.

Carroll 2006 \{published data only\} Carroll KM, Ball SA, Nich C, Martino S, Frankforter TL, Farentinos $\mathrm{C}$, et al. Motivational interviewing to improve treatment engagement and outcome in individuals seeking treatment for substance abuse: a multisite effectiveness study. Drug and Alcohol Dependence 2006;81(3):301-12.
Elliott 2005 \{published data only\}

Elliott L, Glenday J, Freeman L, Ajeda D, Johnston B, Christie $\mathrm{M}$, et al. Reducing diazepam prescribing for illicit drug users: a randomised control study. Drug and Alcohol Review 2005;24(1):25-31.

Elsesser 1996 \{published data only\} Elsesser K, Sartory G, Maurer J. The efficacy of complaints management training in facilitating benzodiazepine withdrawal. Behaviour Research and Therapy 1996;34(2): 149-56.

Gilbert 1993 \{published data only\} Gilbert A, Owen N, Innes J M, Sansom L. Trial of an intervention to reduce chronic benzodiazepine use among residents of aged-care accommodation. Australian and New Zealand Journal of Medicine. 1993;23(4):343-7.

Gosselin 2006 \{published data only\} Gosselin P, Ladouceur R, Morin CM, Dugas MJ, Baillargeon L. Benzodiazepine discontinuation among adults with GAD: a randomized trial of cognitive-behavioral therapy. Journal of Consulting and Clinical Psychology 2006;74(5): 908-19.

Heather 2004 \{published data only\}

Heather N, Bowie A, Ashton H, McAvoy B, Spencer I, Brodie J, et al. Randomised controlled trial of two brief interventions against long-term benzodiazepine use: outcome of intervention. Addiction Research \& Theory 2004;12(2):141-54.

King 2009 \{published data only\} King VL, Stoller KB, Kidorf M, Kindbom K, Hursh S, Brady T, et al. Assessing the effectiveness of an Internetbased videoconferencing platform for delivering intensified substance abuse counseling. Journal of Substance Abuse Treatment 2009;36(3):331-8.

Morin 2004 \{published data only\} Morin CM, Bastien C, Guay B, Radouco-Thomas M, Leblanc J, Vallières A. Randomized clinical trial of supervised tapering and cognitive behavior therapy to facilitate benzodiazepine discontinuation in older adults 
with chronic insomnia. American Journal of Psychiatry 2004; 161(2):332-42.

Nathan 1986 \{published data only\}

Nathan RG, Robinson D, Cherek DR, Sebastian CS, Hack $\mathrm{M}$, Davison S. Alternative treatments for withdrawing the long-term benzodiazepine user: a pilot study. International Journal of the Addictions 1986;21(2):195-211.

O'Connor 2008 \{published data only\}

O'Connor K, Marchand A, Brousseau L, Aardema F, Mainguy N, Landry P, et al. Cognitive-behavioral, pharmacological and psychosocial predictors of outcome during tapered discontinuation of benzodiazepine. Clinical Psychology \& Psychotherapy 2008;15(1):1-14.

Otto 1993 \{published data only\}

Otto MW, Pollack MH, Sachs GS, Reiter SR, MeltzerBrody S, Rosenbaum JF. Discontinuation of benzodiazepine treatment: efficacy of cognitive-behavioral therapy for patients with panic disorder. American Journal of Psychiatry 1993;150(10):1485-90.

Otto 2010 \{published data only\}

Otto MW, McHugh RK, Simon NM, Farach FJ, Worthington JJ, Pollack MH. Efficacy of CBT for benzodiazepine discontinuation in patients with panic disorder: Further evaluation. Behaviour Research and Therapy 2010;48(8):720-7.

Oude Voshaar 2003 a \{published data only\} Oude Voshaar RC, Gorgels WJ, Mol AJ, van Balkom AJ, van de Lisdonk EH, Breteler MHM, et al. Tapering off longterm benzodiazepine use with or without group cognitivebehavioural therapy: Three-conditioned, randomised controlled trial. British Journal of Psychiatry 2003;182(6): 498-504.

Parr 2013 \{published and unpublished data\} Parr JM, Kavanagh DJ, Young RMcD, Stubbs B, Bradizza N. Impact of Cognitive Behaviour Therapy Via Mail for Cessation of Benzodiazepine Use: A Series of Case Reports. Behaviour Change 2013;30(2):74-83.

Scherbaum 2005 \{published data only\} Scherbaum N, Kluwig J, Specka M, Krause D, Merget B, Finkbeiner T, et al. Group psychotherapy for opiate addicts in methadone maintenance treatment - a controlled trial. European Addiction Research 2005;11(4):163-71.

Spiegel 1994 \{published data only\}

Spiegel DA, Bruce TJ, Gregg SF, Nuzzarello A. Does cognitive behavior therapy assist slow-taper alprazolam discontinuation in panic disorder?. American Journal of Psychiatry 1994;151(6):876-81.

Ten Wolde 2008 \{published data only\} Ten Wolde GB, Dijkstra A, van Empelen P, van den Hout W, Neven AK, Zitman F. Long-term effectiveness of computer-generated tailored patient education on benzodiazepines: a randomized controlled trial. Addiction 2008;103(4):662-70.
Vicens 2006 \{published data only\}

Vicens C, Fiol F, Llobera J, Campoamor F, Mateu C, Alegret $S$, et al. Withdrawal from long-term benzodiazepine use: randomised trial in family practice. British Journal of General Practice 2006;56(533):958-63.

Vorma 2002 \{published data only\}

Vorma H, Naukkarinen H, Sarna S, Kuoppasalmi K. Treatment of out-patients with complicated benzodiazepine dependence: comparison of two approaches. Addiction 2002;97(7):851-9.

Zahradnik 2009 \{published data only\} Zahradnik A, Otto C, Crackau B, Löhrmann I, Bischof G, John U, et al. Randomized controlled trial of a brief intervention for problematic prescription drug use in nontreatment-seeking patients. Addiction 2009;104(1):109-17.

\section{References to studies excluded from this review}

Ball 2007 \{published data only\}

Ball SA, Martino S, Nich C, Frankforter TL, Van Horn D, Crits-Christoph P, et al. Site matters: multisite randomized trial of motivational enhancement therapy in community drug abuse clinics. Journal of Consulting and Clinical Psychology 2007;75(4):556-67.

Bélanger 2005 \{published data only\} Bélanger L, Morin CM, Bastien C, Ladouceur R. Selfefficacy and compliance with benzodiazepine taper in older adults with chronic insomnia. Health Psychology 2005;24 (3):281-7.

Bruce 1995 \{published data only\} Bruce TJ, Spiegel DA, Gregg SF, Nuzzarello A. Predictors of alprazolam discontinuation with and without cognitive behavior therapy in panic disorder. American Journal of Psychiatry 1995;152(8):1156-60.

Carroll 2009 \{published data only\} Carroll KM, Martino S, Ball SA, Nich C, Frankforter T, Anez LM, et al. A multisite randomized effectiveness trial of motivational enhancement therapy for Spanish-speaking substance users. Journal of Consulting and Clinical Psychology 2009;77(5):993-9.

\section{Chang 2010 \{published data only\}}

Chang B-H, Sommers E, Herz L. Acupuncture and relaxation response for substance use disorder recovery. Journal of Substance Use 2010;15(6):390-401.

Chutuape 1999 \{published data only\} Chutuape MA, Silverman K, Stitzer M. Contingent reinforcement sustains post-detoxification abstinence from multiple drugs: a preliminary study with methadone patients. Drug and Alcohol Dependence 1999;54(1):69-81.

Cormack 1994 \{published data only\} Cormack MA, Sweeney KG, Hughes-Jones H, Foot GA. Evaluation of an easy, cost effective strategy for cutting benzodiazepine use in general practice. British Journal of General Practice 1994;44(378):5-8.

de Gier 2011 \{published data only\} de Gier NAH, Gorgels WJ, Lucassen PL, Oude Voshaar R, Mulder J, Zitman F. Discontinuation of long-term 
benzodiazepine use: 10-year follow-up. Family Practice 2011;28(3):253-9.

Ghitza 2008 \{published data only\}

Ghitza UE, Epstein DH, Preston KL. Self-report of illicit benzodiazepine use on the Addiction Severity Index predicts treatment outcome. Drug and Alcohol Dependence 2008;97 (1-2):150-7.

\section{Giblin 1983 \{published data only\}}

Giblin MJ, Clift AD. Sleep without drugs. Journal of the Royal College of General Practitioners 1983;33(255):628-33.

Godfrey 2008 \{published data only\}

Godfrey C, Heather N, Bowie A, Brodie J, Parrott S, Ashton H, et al. Randomised controlled trial of two brief interventions against long-term benzodiazepine use: Costeffectiveness. Addiction Research \& Theory 2008;16(4): 309-17.

\section{Heather 2011 \{published data only\}}

Heather N, Paton J, Ashton H. Predictors of response to brief intervention in general practice against long-term benzodiazepine use. Addiction Research \& Theory 2011;19 (6):519-27.

Iguchi 1988 \{published data only\}

Iguchi MY, Stitzer ML, Bigelow GE, Liebson IA. Contingency management in methadone maintenance: effects of reinforcing and aversive consequences on illicit polydrug use. Drug and Alcohol Dependence 1988;22(1-2): $1-7$.

\section{Jones 1990 \{published data only\}}

Jones D. Weaning elderly patients off psychotropic drugs in general practice: a randomised control trial. Health Trends 1990;22:164-6.

Lichstein 2013 \{published data only\} Lichstein KL, Nau SD, Wilson NM, Aguillard RN, Lester KW, Bush AJ, et al. Psychological treatment of hypnoticdependent insomnia in a primarily older adult sample. Behaviour Research and Therapy 2013;51(12):787-96.

Mol 2006 \{published data only\} Mol AJ, Oude Voshaar RC, Gorgels WJ, Breteler MH, van Balkom AJ, van de Lisdonk EH, et al. The absence of benzodiazepine craving in a general practice benzodiazepine discontinuation trial. Addictive Behaviors 2006;31(2): 211-22.

Mol 2007 \{published data only\}

Mol AJ, Oude Voshaar RC, Gorgels WJ, Breteler Marinus $\mathrm{MH}$, van Balkom AJ, van de Lisdonk EH, et al. The role of craving in relapse after discontinuation of long-term benzodiazepine use. Journal of Clinical Psychiatry 2007;68 (12):1894-1900.

\section{Morgan 2004 \{published data only\}}

Morgan K, Dixon S, Mathers N, Thompson J, Tomeny M. Psychological treatment for insomnia in the regulation of long-term hypnotic drug use. Health Technology Assessment (Winchester, England) 2004;8(8):1-68.

Onyett 1988 \{published data only\}

Onyett SR, Turpin G. Benzodiazepine withdrawal in primary care: a comparison of behavioural group training and individual sessions. Behavioural and Cognitive

Psychotherapy 1988;16(4):297-312.

Otto 1992 \{published data only\} Otto MW, Pollack MH, Meltzer-Brody S, Rosenbaum JF. Cognitive-behavioral therapy for benzodiazepine discontinuation in panic disorder patients. Psychopharmacology Bulletin 1992;28(2):123-30.

\section{Oude Voshaar 2003 \{published data only\}}

Oude Voshaar RC, Mol AJ, Gorgels WJ, Breteler MH, van Balkom AJ, van de Lisdonk EH, et al. Cross-validation, predictive validity, and time course of the Benzodiazepine Dependence Self-Report Questionnaire in a benzodiazepine discontinuation trial. Comprehensive Psychiatry 2003;44(3): 247-55.

Oude Voshaar 2006a \{published data only\} Oude Voshaar RC, Gorgels WJ, Mol AJ, van Balkom AJ, Mulder J, van de Lisdonk EH, et al. Predictors of long-term benzodiazepine abstinence in participants of a randomized controlled benzodiazepine withdrawal program. Canadian Journal of Psychiatry. Revue Canadienne De Psychiatrie 2006; 51(7):445-52.

Oude Voshaar 2006b \{published data only\} Oude Voshaar RC, Krabbe PFM, Gorgels WJ, Adang EM, van Balkom AJ, van de Lisdonk EH, et al. Tapering off benzodiazepines in long-term users - an economic evaluation. Pharmacoeconomics 2006;24(7):683-94.

Pollack 2002 \{published data only\} Pollack MH, Penava SA, Bolton E, Worthington JJ 3rd, Allen GL, Farach FJ Jr, et al. A novel cognitive-behavioral approach for treatment-resistant drug dependence. Journal of Substance Abuse Treatment 2002;23(4):335-42.

Salonoja 2010 \{published data only\} Salonoja M, Salminen M, Aarnio P, Vahlberg T, Kivelä S-L. One-time counselling decreases the use of benzodiazepines and related drugs among community-dwelling older persons. Age and Ageing 2010;39(3):313-9.

Scherzer 1996 \{published data only\} Scherzer BR. Biofeedback-Assisted Cognitive-Behavioral Therapy in the Reduction of Alprazolam Dependence after Panic Attacks with Cardiaphobia [PhD thesis]. Ohio, USA: The Union Institute, 1996:100.

Soeffing 2008 \{published data only\} Soeffing JP, Lichstein KL, Nau SD, McCrae CS, Wilson NM, Aguillard RN, et al. Psychological treatment of insomnia in hypnotic-dependent older adults. Sleep Medicine 2008;9(2):165-71.

Stitzer 1992 \{published data only\} Stitzer ML, Iguchi MY, Felch LJ. Contingent take-home incentive: Effects on drug use of methadone maintenance patients. Journal of Consulting and Clinical Psychology 1992; 60(6):927-34.

Taylor 2010 \{published data only\} Taylor DJ, Schmidt-Nowara W, Jessop CA, Ahearn J. Sleep restriction therapy and hypnotic withdrawal versus sleep 
hygiene education in hypnotic using patients with insomnia. Journal of Clinical Sleep Medicine 2010;6(2):169-75.

\section{Vorma 2005 \{published data only\}}

Vorma H, Naukkarinen HH, Sarna SJ, Kuoppasalmi KI. Predictors of benzodiazepine discontinuation in subjects manifesting complicated dependence. Substance Use \& Misuse 2005;40(4):499-510.

\section{Additional references}

\section{Amato 2011a}

Amato L, Minozzi S, Davoli M, Vecchi S. Psychosocial and pharmacological treatments versus pharmacological treatments for opioid detoxification. Cochrane Database of Systematic Reviews 2011, Issue 9. [DOI: 10.1002/ 14651858.CD005031.pub4]

\section{Amato 2011b}

Amato L, Minozzi S, Davoli M, Vecchi S. Psychosocial combined with agonist maintenance treatments versus agonist maintenance treatments alone for treatment of opioid dependence. Cochrane Database of Systematic Reviews 2011, Issue 10. [DOI: 10.1002/ 14651858.CD004147.pub4]

\section{Backmund 2005}

Backmund M, Meyer K, Henkel C, Soyka M, Reimer J, Schütz CG. Co-consumption of benzodiazepines in heroin users, methadone-substituted and codeine-substituted patients. Journal of Addictive Diseases 2005;24(4):17-29.

\section{Barker 2004}

Barker MJ, Greenwood KM, Jackson M, Crowe SF. Cognitive effects of long-term benzodiazepine use: a metaanalysis. CNS Drugs 2004;18(1):37-48.

\section{Bartlett 2009}

Bartlett G, Abrahamowicz M, Grad R, Sylvestre MP, Tamblyn R. Association between risk factors for injurious falls and new benzodiazepine prescribing in elderly patients. BMC Family Practice 2009;10:1. [DOI: 10.1186/ 1471-2296-10-1]

\section{Beck 1993}

Beck A. Cognitive therapy: past, present, and future. Journal of Consulting and Clinical Psychology 1993;61(2): 194-8.

Billioti de Gage 2012 Billiot de Gage S, Bégaud B, Bazin F, Verdoux H, Dartigues JF, Pérès K, et al. Benzodiazepine use and risk of dementia: prospective population based study. BMJ 2012;345:e6231.

\section{Bleich 1999}

Bleich A, Gelkopf M, Schmidt V, Hayward R, Bodner $\mathrm{G}$, Adelson M. Correlates of benzodiazepine abuse in methadone maintenance treatment. A 1 year prospective study in Israeli clinic. Addiction 1999;94(10):1533-40.

\section{British National Formulary 2010}

British Medical Association and Royal Pharmaceutical Association. British National Formulary 60. British Medical Journal Publishing Group and Pharmaceutical Press, September 2010.

\section{Bruce 1999}

Bruce TJ, Spiegel DA, Hegel MT. Cognitive-behavioral therapy helps prevent relapse and recurrence of panic disorder following alprazolam discontinuation: a long-term follow-up pf the Peoria and Dartmouth studies. Journal of Consulting and Clinical Psychology 1999;67(1):151-6.

\section{Brunette 2004}

Brunette MF, Muesser KT, Drake RE. A review of research on residential programs for people with severe mental illness and co-occurring substance use disorders. Drug and Alcohol Review 2004;23(4):471-81.

\section{Carroll 1996}

Carroll KM. Relapse prevention as a psychosocial treatment approach: a review of controlled clinical trials. Experimental and Clinical Psychopharmacology 1996;4:46-54.

\section{Chutuape 1997}

Chutuape MA, Brooner RK, Stitzer M. Sedative use disorders in opiate-dependent patients: association with psychiatric and other substance use disorders. Journal of Nervous Mental Disorders 1997;185(5):289-97.

\section{Darke 1993}

Darke S, Swift W, Hall W, Ross M. Drug use, HIV risktaking and psychosocial correlates of benzodiazepine use among methadone maintenance clients. Drug and Alcohol Dependence 1993;34(1):67-70.

\section{Darke 1994}

Darke S, Swift W, Hall W. Prevalence, severity and correlates of psychological morbidity among methadone maintenance patients. Addiction 1994;89(2):211-7.

\section{Denis 2006}

Denis C, Lavie E, Fatséas M, Auriacombe M.

Psychotherapeutic interventions for cannabis abuse and/ or dependence in outpatient settings. Cochrane Database of Systematic Reviews 2006, Issue 3. [DOI: 10.1002/ 14651858.CD005336.pub2]

\section{Department of Health \& Children 2002}

Department of Health \& Children. Benzodiazepines: good practice guidelines for clinicians. 2002. http:// www.drugsandalcohol.ie/5349/1/1500-1388.pdf Accessed 20 October 2013.

\section{DSM-IV 1994}

American Psychiatric Association. Diagnostic and Statistical Manual of Mental Disorders: DSM-IV. Washington DC: American Psychiatric Association, 1994:181-83.

\section{Fals-Stewart 2005}

Fals-Stewart W, O'Farrell TJ, Birchler GR, Córdova J, Kelley MC. Behavioral couples therapy for alcoholism and drug abuse: where we've been, where we are and where we are going. Journal of Cognitive Psychotherapy 2005;19(3): $229-46$.

Ferri 2006

Ferri M, Amato L, Davoli M. Alcoholics Anonymous and other 12-step programmes for alcohol dependence. Cochrane Database of Systematic Reviews 2006, Issue 3. [DOI: 10.1002/14651858.CD005032.pub2] 


\section{Gould 2014a}

Gould RL, Coulson MC, Patel N, Highton-Williamson E, Howard RJ. Interventions for reducing benzodiazepine use in older people: meta-analysis of randomised controlled trials. British Journal of Psychiatry 2014;204(2):98-107.

\section{Gould 2014b}

Gould RL, Coulson MC, Highton-Williamson E, Howard RJ. Interventions for reducing benzodiazepine use in older people: meta-analysis of randomised controlled trials. Erratum. British Journal of Psychiatry 2014;205(4):330.

\section{Higgins 2011}

Higgins JPT, Green S (editors). Cochrane Handbook for Systematic Reviews of Interventions Version 5.1.0 [updated March 2011]. The Cochrane Collaboration, 2011. Available from www.cochrane-handbook.org.

\section{Jones 2012}

Jones JD, Mogali S, Comer SD. Polydrug abuse: a review of opioid and benzodiazepine combination use. Drug and Alcohol Dependence 2012;125(1-2):8-18.

\section{Kaner 2007}

Kaner EFS, Dickinson HO, Beyer FR, Campbell F, Schlesinger C, Heather N, et al. Effectiveness of brief alcohol interventions in primary care populations. Cochrane Database of Systematic Reviews 2007, Issue 2. [DOI: 10.1002/14651858.CD004148.pub3]

\section{Knapp 2007}

Knapp WP, Soares B, Farrell M, Silva de Lima M. Psychosocial interventions for cocaine and psychostimulant amphetamines related disorders. Cochrane Database of Systematic Reviews 2007, Issue 3. [DOI: 10.1002/ 14651858.CD003023.pub2]

\section{Lefebvre 2011}

Lefebvre C, Manheimer E, Glanville J, on behalf of the Cochrane Information Retrieval Methods Group. Chapter 6: Searching for studies. In: Higgins JPT, Green S (editors). Cochrane Handbook for Systematic Reviews of Interventions Version 5.1.0 [updated March 2011]. The Cochrane Collaboration, 2011. Available from www.cochrane-handbook.org.

Lui 2008

Lui S, Terplan M, Smith EJ. Psychosocial interventions for women enrolled in alcohol treatment during pregnancy. Cochrane Database of Systematic Reviews 2008, Issue 3. [DOI: 10.1002/14651858.CD006753.pub2]

\section{Marlatt 1985}

Marlatt GA, Gordon JR. Relapse Prevention: Maintenance Strategies in the Treatment of Addictive Behaviors. New York: Guilford Press, 1985.

\section{Mayet 2005}

Mayet S, Farrell M, Ferri M, Amato L, Davoli M. Psychosocial treatment for opiate abuse and dependence. Cochrane Database of Systematic Reviews 2005, Issue 1. [DOI: 10.1002/14651858.CD004330.pub2]

\section{McQueen 2009}

McQueen J, Howe TE, Allan L, Mains D. Brief interventions for heavy alcohol users admitted to general hospital wards. Cochrane Database of Systematic Reviews 2009, Issue 3. [DOI: 10.1002/14651858.CD005191.pub2]

\section{Miller 1991}

Miller WR, Rollnick S. Motivational Interviewing: Preparing People for Change. 1st Edition. New York: Guilford Press, 1991.

\section{Miller 1999}

Miller WR, Meyers RJ, Hiller-Sturmhöfel S. The community-reinforcement approach. Alcohol Research \& Health 1999;23(2):116-21.

\section{Morin 2005}

Morin CM, Bélanger L, Bastien C, Vallières A. Longterm outcome after discontinuation of benzodiazepines for insomnia: a survival analysis of relapse. Behaviour Research and Therapy 2005;43(1):1-14.

\section{Mugunthan 2011}

Mugunthan K, McGuire T, Glasziou P. Minimal interventions to decrease long-term use of benzodiazepines in primary care: a systematic review and meta-analysis. British Journal of General Practice 2011;61(590):e573-8.

\section{NICE 2011}

National Institute for Health and Care Excellence. CG113 Anxiety: NICE Guidance. January 2011. http:// guidance.nice.org.uk/CG113/NICEGuidance/pdf/English (accessed 20 May 2011).

\section{Noyes 1988}

Noyes R Jr, Garvey MJ, Cook BL, Perry PJ. Benzodiazepine withdrawal: a review of the evidence. Journal of Clinical Psychiatry 1988;49(10):382-9.

\section{O'Brien 2005}

O'Brien CP. Benzodiazepine use, abuse, and dependence. Journal of Clinical Psychiatry 2005;66(Suppl 2):28-33.

Oliver 2007

Oliver P, Forrest R, Keen J. Benzodiazepines and cocaine as risk factors in fatal opioid overdoses. National Treatment Agency for Substance Misuse, Research Briefing: 31. April 2007. http://www.nta.nhs.uk/uploads/

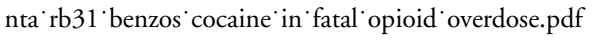
Accessed 20 October 2013:1-20.

\section{Onken 1990}

Onken LS, Blaine JD. Psychotherapy and counselling research in drug abuse treatment: questions, problems and solutions. In: Onken LS, Blaine JD, Rockville MD editor (s). Psychotherapy and Counseling in the Treatment of Drug Abuse (NIDA Research Monograph 104). Rockville, MD: National Institute on Drug Abuse, 1990:1-5.

Otto 2009

Otto C, Crackau B, Löhrmann I, Zahradnik A, Gallus B, John $\mathrm{U}$, et al. Brief intervention in general hospital for problematic prescription drug use: 12-month outcome. Drug and Alcohol Dependence 2009;105(3):221-6. 
Oude Voshaar 2006c

Oude Voshaar RC, Couvée JE, Van Balkom AJLM, Mulder PGH, Zitman FG. Strategies for discontinuing long-term benzodiazepine use: meta-analysis. British Journal of Psychiatry 2006;189:213-20.

Oude Voshaar 2006d

Oude Voshaar RC, Gorgels WJMJ, Mol AJJ, van Balko AJLM, Mulder J, van de Lisdonk EH, et al. Long-term outcome of two forms of randomised benzodiazepine discontinuation. British Journal of Psychiatry 2006;188: $188-9$.

Parr 2008

Parr JM, Kavanagh DJ, Cahill L, Mitchell G, McD Young R. Effectiveness of current treatment approaches for benzodiazepine discontinuation: a meta-analysis. Addiction 2008;104(1):13-24

\section{Petursson 1993}

Petursson H, Gudjonsson GH, Lader MH. Psychometric performance during withdrawal from long-term benzodiazepine treatment. Psychopharmacology 1993;81(4): 345-9.

\section{RevMan 2014}

The Nordic Cochrane Centre, The Cochrane Collaboration. Review Manager (RevMan). 5.3. Copenhagen: The Nordic Cochrane Centre, The Cochrane Collaboration, 2014.

Rounsaville 1983

Rounsaville BJ, Glazer W, Wilber CH, Weissman MM, Kleber HD. Short-term interpersonal psychotherapy in methadone-maintained opiate addicts. Archives of General Psychiatry 1983;40(6):629-36.

\section{SAMHSA 1999}

Substance Abuse and Mental Health Services

Administration. Brief interventions in substance abuse treatment. Brief Interventions and Brief Therapies for Substance Abuse: TIP 34. Rockville, MD: US Department of Health and Human Services, 1999

\section{SAMHSA 2014}

Substance Abuse and Mental Health Service Administration. Screening, brief intervention and referral to treatment. 2014. http://www.samhsa.gov/sbirt Accessed 20 October 2013.

Smink 2010

Smink BE, Egberts AC, Lusthof KJ, Uges DR, de Gier JJ. The relationship between benzodiazepine use and traffic accidents: a systematic review. CNS Drugs 2010;24(8): 639-53.

Smith 2010

Smith AJ, Tett SE. Improving the use of benzodiazepines is it possible? A non-systematic review of interventions tried in the last 20 years. BMC Health Services Research 2010;10 (321): $1-12$.

\section{Stitzer 2006}

Stitzer M, Petry N. Contingency management for treatment of substance abuse. Annual Review of Clinical Psychology 2006;2:411-34.

\section{Tyrer 2010}

Tyrer P. Benzodiazepine substitution for dependent patients - going with the flow. Addiction 2010;105(11):1875-8.

\section{UKATT 2001}

UKATT Research Team. United Kingdom Alcohol

Treatment Trial (UKATT): hypotheses, design and methods. Alcohol and Alcoholism 2001;36(1):11-21.

van den Hoek 1990

van den Hoek JAR, van Haarstrecht HJA, Goudsmit J, de Wolf F, Coutinho RA. Prevalence, incidence and risk factors of hepatitis $C$ virus infection among drug users in Amsterdam. Journal of Infectious Disease 1990;162(4): 823-6.

Vicens 2008

Vicens Caldentey C, Fiol Gelabert F, González Garrido E, Martínez Ojeda E, Mateu Sabater C, Llobera Cánaves J. Long-term effectiveness of an intervention to discontinue chronic benzodiazepine use [Spanish]. Actas Españolas de Psiquiatría 2008;36(5):295-8.

Vorma 2003

Vorma H, Naukkarinen H, Sarna S, Kuoppasalmi K. Longterm outcome after benzodiazepine withdrawal treatment in subjects with complicated dependence. Drug and Alcohol Dependence 2003;70(3):309-14.

Wagner 2004

Wagner AK, Zhang F, Soumorai SB, Walker AM, Gurwitz $\mathrm{JH}$, Glynn RJ, et al. Benzodiazepine use and hip fractures in the elderly: who is at greatest risk?. Archives of Internal Medicine 2004;164(14):1567-72.

\section{WHO 2009a}

World Health Organization. International Statistical Classification of Disease and Related Health Problems. 10th Edition. Washington DC: World Health Organization, 2009.

\section{WHO 2009b}

World Health Organization. Guidelines for the psychosocially assisted pharmacological treatment of opioid dependence. 2009. http://www.who.int/substance abuse/ activities/treatment' opioid 'dependence/en/index.html (accessed 9 January 2012).

\section{WHO 2010}

WHO. Management of substance abuse. Lexicon of terminology. 2010. http://www.who.int/substance abuse/ terminology/who lexicon/en/ (accessed 20 May 2010).

\section{References to other published versions of this review}

\section{Darker 2012}

Darker CD, Sweeney BP, Barry JM, Farrell MF. Psychosocial interventions for benzodiazepine harmful use, abuse or dependence. Cochrane Database of Systematic Reviews 2012, Issue 2. [DOI: 10.1002/14651858.CD009652]

* Indicates the major publication for the study 


\section{CHARACTERISTICS OF STUDIES}

\section{Characteristics of included studies [ordered by study ID]}

Bagøien 2013

\begin{tabular}{|c|c|}
\hline Methods & RCT. \\
\hline Participants & $\begin{array}{l}\text { Patients attending an emergency psychiatric in-patient service in Norway. } 135 \text { patients } \\
\text { in trial overall. } 7 \text { patients classified as using BZDs; } 4 \text { control condition, } 3 \text { experimental } \\
\text { condition. Mean age } 47.5 \text { years }(S D=19.7) \text { for control, } 45.7 \text { years }(S D=17.2) \text { experi- } \\
\text { mental. ICD- } 10 \text { classification for psychoactive substance use were used for some patients } \\
\text { but not all }\end{array}$ \\
\hline Interventions & $\begin{array}{l}\text { Intervention group: MI. The intervention consisted of } 2 \text { sessions of manual guided MI } \\
\text { delivered individually to the patients by a trained therapist. The manual was developed } \\
\text { by two MI trainers in co-operation with the first author of this manuscript. Each session } \\
\text { was planned to last } 45 \text { minutes. Depending on the patient' length of stay in the hospital, } \\
\text { the second session took place on another day or later the same day } \\
\text { In the first session the patients' ambivalence to substance use was explored. Also the } \\
\text { severity of the patients' substance use was considered. In the second session the patients' } \\
\text { experiences of substance use and prior attempts to change were explored to build intrinsic } \\
\text { motivation for change. Actual readiness for change in substance use patterns and com- } \\
\text { mitment to a change plan were focused on. The intervention was delivered in a MI style. } \\
\text { If they wanted, patients received information about, and referral to available follow-up } \\
\text { treatment programs for substance use. The interviewer offered a written summary from } \\
\text { the } 2 \text { sessions to each patient } \\
\text { Control group: TAU. TAU was individualized according to the clinical condition of } \\
\text { the patients during the stay and in accordance with general national and international } \\
\text { medical standards. It would usually include detoxification, pharmacotherapy and general } \\
\text { psychotherapy. Also, treatment would be given for any coexisting non-substance-related } \\
\text { disorder, including psychiatric disorders } \\
\text { General information about the harmful effects of substances and suggestions regarding } \\
\text { treatment for substance use, including possible referral to specialty substance use treat- } \\
\text { ment institutions, would be given. Planning of discharge with referral to out-patient and } \\
\text { primary community health care after discharge usually would be included }\end{array}$ \\
\hline
\end{tabular}
Urinalysis used at baseline but not for follow-up time points

$\begin{array}{ll}\text { Notes } & \text { Funding source: St. Olav Universoty Hospital, Trondheim, Norway } \\ \text { Declaration of interest: None. }\end{array}$

\section{Risk of bias}

\section{Bias} Authors' judgement Support for judgement

Random sequence generation (selection Low risk bias)
"Randomisation was performed by a web-based system developed and administered by the Unit of Applied Clinical Research, Institute of Cancer Research and Molecular Medicine, Norwe- 
Bagøien 2013 (Continued)

\begin{tabular}{|c|c|c|}
\hline & & $\begin{array}{l}\text { gian University of Science and Technology, Trondheim, Nor- } \\
\text { way. This was a block randomisation, with the block size for } \\
\text { all } 3 \text { strata set to } 10 \text { in each strata group. The randomisation } \\
\text { logarithm was programmed in PHP with a My SQL database" }\end{array}$ \\
\hline Allocation concealment (selection bias) & Low risk & $\begin{array}{l}\text { "The clinicians making the baseline assessments had no infor- } \\
\text { mation regarding the block size used for randomisation" }\end{array}$ \\
\hline $\begin{array}{l}\text { Blinding (performance bias and detection } \\
\text { bias) } \\
\text { All outcomes }\end{array}$ & Unclear risk & No objective measures used. \\
\hline $\begin{array}{l}\text { Blinding of participants and personnel } \\
\text { (performance bias) } \\
\text { All outcomes }\end{array}$ & High risk & $\begin{array}{l}\text { No blinding or incomplete blinding, and the outcome is likely } \\
\text { to be influenced by lack of blinding }\end{array}$ \\
\hline $\begin{array}{l}\text { Blinding of outcome assessment (detection } \\
\text { bias) } \\
\text { All outcomes }\end{array}$ & Unclear risk & No objective measures used. \\
\hline $\begin{array}{l}\text { Blinding of outcome assessor (detection } \\
\text { bias) subjective outcomes }\end{array}$ & High risk & $\begin{array}{l}\text { Patients were not blinded to allocation and patients were self- } \\
\text { reporting the data. However if patients were late returning the } \\
\text { questionnaire then a nurse blinded to allocation phoned them. } \\
\text { "If we did not receive the questionnaire during the following } 14 \\
\text { days, nurses from the department, blind to treatment allocation, } \\
\text { made telephone calls to ask for patients' reply" }\end{array}$ \\
\hline $\begin{array}{l}\text { Incomplete outcome data (attrition bias) } \\
\text { All outcomes }\end{array}$ & High risk & $\begin{array}{l}\text { Authors reported } 46 \% \text { loss to follow-up. To partially compensate } \\
\text { for this they applied a regression model which was deemed less } \\
\text { susceptible to bias under the assumption of missing data }\end{array}$ \\
\hline Selective reporting (reporting bias) & Low risk & $\begin{array}{l}\text { The study protocol is not available, but the published reports } \\
\text { include all expected outcomes, including those that were pre- } \\
\text { specified in the method section }\end{array}$ \\
\hline
\end{tabular}

Baillargeon 2003

\begin{tabular}{ll}
\hline Methods & RCT. \\
\hline Participants & 65 people aged over 50 with chronic $(>6$ months) insomnia who had been taking BZDs \\
& $\begin{array}{l}\text { every night for }>3 \text { months. Recruited through media advertisements or referred by their } \\
\text { GP in Canada. } 35 \text { participants }(21 ; 60 \% \text { female) in the intervention condition (mean } \\
\text { age }=68.3, \mathrm{SD}=7.4) ; 30 \text { participants }(17 ; 57 \% \text { female) in control condition (mean age } \\
=66.4, \mathrm{SD}=6.0)\end{array}$
\end{tabular}

Interventions

Intervention group: CBT plus tapering. CBT involved behavioural, cognitive and educational components. The behavioural component included instructions for stimulus control and procedures for sleep restriction. The cognitive component addressed irrational thinking. The educational component included sleep hygiene education and in-

Psychosocial interventions for benzodiazepine harmful use, abuse or dependence (Review) 
Control group: Tapering supervised by a physician weekly over 8 weeks

Outcomes

BZD discontinuation, confirmed by blood screening performed at each of 3 measurement points (immediately after treatment completion and at 3- and 12-month followup)

Notes

Funding source: Author RV's work was supported by Laval University Chair for Geriatric Research. Grant received from the National Health Research and Development Program, Health Canada (6605-4573-702)

Declaration of interest: None declared.

\section{Risk of bias}

\section{Bias}

Random sequence generation (selection Low risk bias)

Allocation concealment (selection bias) Unclear risk

ding (performance bias and detection Low risk bias)

All outcomes

Blinding of participants and personnel High risk (performance bias)

All outcomes

Blinding of outcome assessment (detection Low risk bias)

All outcomes

Blinding of outcome assessor (detection High risk bias) subjective outcomes

Incomplete outcome data (attrition bias) Low risk All outcomes
Table of random numbers, arranged by a nurse.

"The treatment assignment could not be concealed from participants, but aggregate outcome data were not revealed to patients or investigators during the study"

While the treatment providers were in regular contact with the patients the method of ascertainment (blood screening) of the objective outcome was not susceptible to bias

"At each visit the physician looked for withdrawal symptoms and prescribed either the same or a lower dosage, depending on the patient's symptoms"

"The main outcome measure was benzodiazepine discontinuation, confirmed by blood screening"

No blinding reported. Subjective reports ("benzodiazepine consumption and sleep measures were evaluated by means of the sleep diary completed by participants") could have been influenced

No missing data.
The study protocol is not available but the published report includes the expected outcome which was pre-specified in the methods section 


\begin{tabular}{|c|c|}
\hline Methods & RCT. \\
\hline Participants & $\begin{array}{l}\text { Participants were } 214 \text { regular users of amphetamines recruited from the Newcastle region } \\
(\mathrm{n}=98) \text { of NSW and from the Greater Brisbane Region of South-East Queensland ( } \mathrm{n}= \\
\text { 116), Australia. Enrolled in pharmacotherapy for heroin dependence. BZD use amongst } \\
\text { the cohort. Data specifically relating to participants using BZDs was supplied by author } \\
-17 \text { participants in the intervention group 1; } 14 \text { participants in the intervention group } \\
\text { 2; } 12 \text { participants control condition }\end{array}$ \\
\hline Interventions & $\begin{array}{l}\text { Intervention group 1: } 2 \text { sessions of CBT, plus self-help booklet. "The procedure and } \\
\text { content of the first two sessions was the same as described above for the longer interven- } \\
\text { tion" } \\
\text { Intervention group 2: } 4 \text { sessions of CBT, plus self-help booklet. "A therapist manual } \\
\text { revised and a self-help booklet guided treatment sessions, which focused on developing } \\
\text { skills to reduce amphetamine use. Sessions were conducted individually and lasted } 45- \\
60 \text { minutes. Session content included role-plays and take-home exercises for practising } \\
\text { skills. The first session involved a motivational interview to increase motivation to re- } \\
\text { duce amphetamine use. The following sessions focused on cognitive-behavioural coping } \\
\text { strategies and relapse prevention. In the second session, participants were taught how } \\
\text { to reduce craving with progressive muscular relaxation and coping self-talk. The third } \\
\text { session focused on controlling thoughts about using amphetamine. The fourth session } \\
\text { focused on coping with lapses and developing a coping drill to use in high-risk situations } \\
\text { following any future lapses" } \\
\text { Control group: Self-help booklet only. }\end{array}$ \\
\hline
\end{tabular}

Outcomes
Self-reported BZD use, mental health measures and risk taking. Measures were taken at baseline, 5 weeks post treatment, and 6 month follow-up

Additional data supplied by author.

Review group took the decision to look at intervention 1 (17 participants) versus control (12 participants) $\mathrm{N}=29$

Funding source: Commonwealth Department of Health \& Ageing.

Declaration of interest: Not reported.

\section{Risk of bias}

Bias

Authors' judgement Support for judgement

Random sequence generation (selection Low risk bias)

Allocation concealment (selection bias) Low risk

Blinding (performance bias and detection Unclear risk bias)

All outcomes
"A nine-block randomisation schedule was used, which was coordinated by an independent clinical trials researcher"

"A nine-block randomisation schedule was used, which was coordinated by an independent clinical trials researcher"

No objective measures used.

Note: Urine toxicology performed on random $20 \%$ of sample for amphetamine use, but not for BZD use. 
Blinding of participants and personnel High risk (performance bias)

All outcomes

Blinding of outcome assessment (detection Unclear risk bias)

All outcomes

Blinding of outcome assessor (detection Low risk bias) subjective outcomes

Incomplete outcome data (attrition bias) Low risk All outcomes

Selective reporting (reporting bias) Low risk
No blinding or incomplete blinding, and the outcome is likely to be influenced by lack of blinding
No objective measures used.

Note: Urine toxicology performed on random $20 \%$ of sample for amphetamine use, but not for BZD use.

Assessments were conducted by trained interviewers who were blind to participants' treatment allocation

ITT analyses performed.

Sever

The study protocol is available and all of the study's pre-specified (primary and secondary) outcomes that are of interest in the review have been reported as outlined in the protocol

Becka 2004

\begin{tabular}{ll}
\hline Methods & RCT. \\
\hline Participants & $\begin{array}{l}\text { Opioid dependent patients from MMT treatment in Czech Republic that in last } 1 \text { to } 3 \\
\text { months had at least two positive urine toxicological examinations for amphetamine or } \\
\text { BZDs, or both, and at the same time admitted to have amphetamine or BZDs abuse } \\
\text { problem, or both. N=16 patients in intervention group (11 male; } 5 \text { female). } \mathrm{N}=15 \\
\text { patients in control group (12 male; } 3 \text { female) }\end{array}$ \\
\hline
\end{tabular}

Interventions $\quad$ Intervention group: Five sessions once a week for six weeks which included MI, cognitive behavioural assessment, dealing with drug-use antecedents, dealing with drug cravings, systematic self-rewording for achieved results

Control group: Standard methadone substitution treatment.

Outcomes

Use of amphetamine or BZDs, or both, (urine drug screen) at baseline and monthly for 3 months

Notes Additional data supplied by author.

Funding source: Not reported.

Declaration of interest: Not reported.

\section{Risk of bias}

\section{Bias}

Random sequence generation (selection High risk bias)

Allocation concealment (selection bias)
High risk 
Becka 2004 (Continued)

\begin{tabular}{l|l|l}
\hline $\begin{array}{l}\text { Blinding (performance bias and detection } \\
\text { bias) } \\
\text { All outcomes }\end{array}$ & Unclear risk & Urintoxicology. \\
\hline $\begin{array}{l}\text { Blinding of participants and personnel } \\
\text { (performance bias) } \\
\text { All outcomes }\end{array}$ & Unclear risk & No subjective measures used. \\
\hline $\begin{array}{l}\text { Blinding of outcome assessment (detection } \\
\text { bias) } \\
\text { All outcomes }\end{array}$ & Low risk & Urintoxicology. \\
\hline $\begin{array}{l}\text { Blinding of outcome assessor (detection } \\
\text { bias) subjective outcomes }\end{array}$ & Unclear risk & No subjective measures were used. \\
\hline $\begin{array}{l}\text { Incomplete outcome data (attrition bias) } \\
\text { All outcomes }\end{array}$ & Low risk & No missing data. \\
\hline \begin{tabular}{l} 
Selective reporting (reporting bias) \\
\hline
\end{tabular} & Low risk & $\begin{array}{l}\text { The study protocol is not available but the published reports } \\
\text { include all expected outcomes, including those that were pre- } \\
\text { specified in the method section }\end{array}$ \\
\hline
\end{tabular}

Belleville 2007

\begin{tabular}{ll}
\hline Methods & RCT. \\
\hline Participants & 53 BZD and z-hypnotic using chronic insomniac patients $(34$ women; 19 men $)$ in \\
& $\begin{array}{l}\text { Canada. The mean age was } 55.3 \text { years }(\mathrm{CSD}=11.4) \\
\mathrm{N}=28 \text { patients in intervention group }(10 \text { male; } 18 \text { female }) . \mathrm{N}=25 \text { patients in control } \\
\text { group }(9 \text { male; } 16 \text { female })\end{array}$ \\
\hline
\end{tabular}

Interventions

Intervention group: CBT self-help manual, plus medically supervised taper. Patients could ask questions related to the CBT material given 5 booklets $\mathrm{x} 15$ pages each sent 1 a week for the first 5 weeks. They included behavioural and cognitive components Control group: Taper under medical supervision. Step by step withdrawal schedule with plan to discontinue hypnotic by week 8 , including transfer to single hypnotic and $25 \%$ reduction every 2 weeks. 20 page booklet given to all participants on how to manage withdrawal was given. Meeting with physician week 1 and week 4 or 5 to address withdrawal symptoms

Both the Intervention and the Control groups were called by therapists at home once a week and given support and encouragement including adjustment of taper schedule

Outcomes

Use of hypnotic medication dosage or discontinuation measured at baseline, post-treatment, 1 month, 3 months and 6 months follow-up

Notes

z-hypnotics and BZD were disaggregated by contacting author.

Funding source: Not reported.

Declaration of interest: Not reported.

Psychosocial interventions for benzodiazepine harmful use, abuse or dependence (Review)

Copyright @ 2015 The Cochrane Collaboration. Published by John Wiley \& Sons, Ltd. 
Belleville 2007 (Continued)

\section{Risk of bias}

\begin{tabular}{l|l|l}
\hline Bias & Authors' judgement & Support for judgement \\
\hline $\begin{array}{l}\text { Random sequence generation (selection } \\
\text { bias) }\end{array}$ & Low risk & $\begin{array}{l}\text { Patients matched for type of hypnotic (BZD versus non-BZD) } \\
\text { and randomised by one of the authors using sequence generated } \\
\text { by a online random numbers generator }\end{array}$ \\
\hline $\begin{array}{l}\text { Allocation concealment (selection bias) } \\
\begin{array}{l}\text { Blinding (performance bias and detection } \\
\text { bias) } \\
\text { All outcomes }\end{array}\end{array}$ & High risk & Allocation was not concealed. \\
\hline
\end{tabular}

Blinding of participants and personnel High risk (performance bias)

No blinding and subjective outcomes likely to have been influ-

All outcomes enced

Blinding of outcome assessment (detection Unclear risk bias)

No objective outcomes were used.

All outcomes

Blinding of outcome assessor (detection High risk bias) subjective outcomes

No blinding and subjective outcomes likely to have been influenced

Incomplete outcome data (attrition bias) Low risk

All outcomes

"All available data from dropped out participants or participants who did not complete all of the follow-up evaluations were kept in the statistical analyses to preserve the initial composition of the randomized samples"

Selective reporting (reporting bias) Low risk

The study protocol is not available but the published report includes the expected outcomes which were pre-specified in the methods section

\section{Carroll 2006}

\begin{tabular}{ll}
\hline Methods & RCT. \\
\hline Participants & $\begin{array}{l}\text { Patients seeking treatment for a substance use problem in USA. Author was contacted to } \\
\text { provide data that has been disaggregated to identify BZD participants only. This resulted } \\
\text { in } \mathrm{N}=3 \text { for BZD patients. } \mathrm{N}=1 \text { patient in intervention group; } \mathrm{N}=2 \text { patients in control } \\
\text { group }\end{array}$
\end{tabular}

Interventions

Intervention group: MI. Individuals assigned to this condition participated in an approximately 2 hour assessment/evaluation session within which the therapist conducted the same intake/orientation session as described above, but did so in a manner that incorporated MI strategies (e.g. practicing empathy, providing choice, removing barriers, providing feedback and clarifying goals) and that used an MI interviewing style (e.g. 


\section{Carroll 2006 (Continued)}

\begin{tabular}{|c|c|}
\hline & $\begin{array}{l}\text { asking open-ended questions, listening reflectively, affirming change-related participant } \\
\text { statements and efforts, eliciting self-motivational statements with directive methods, and } \\
\text { handling resistance without direct confrontation). A detailed manual was developed for } \\
\text { this protocol that drew from existing MI manuals and guides and adapted them to be } \\
\text { used in the single-session format and which anticipated a participant sample with a wide } \\
\text { range of substance use problems } \\
\text { Control group: Standard intake/evaluation session. Participants assigned to this condi- } \\
\text { tion received an approximately } 2 \text { hour assessment/evaluation session during which the } \\
\text { clinician collected standard information according to their agency guidelines. This typ- } \\
\text { ically included collecting information on the participant's history and current level of } \\
\text { substance use, treatment history and psychosocial functioning; the clinician then pro- } \\
\text { vided an orientation to the clinic. Following this single protocol session, the participant } \\
\text { was referred to standard group treatment at each site. In some cases, groups were led by } \\
\text { the clinician who provided the protocol session but in most cases were led by other staff } \\
\text { at the clinic }\end{array}$ \\
\hline Outcomes & $\begin{array}{l}\text { Urinanlysis and self-reported drug use at baseline, } 28 \text { days post baseline and } 84 \text { days post } \\
\text { baseline }\end{array}$ \\
\hline Notes & $\begin{array}{l}\text { Disaggregated data. } \\
\text { Funding source: NIDA as part of the Cooperative Agreement on National Drug Abuse } \\
\text { Treatment Clinical Trials Network (CTN) U10 numbers (DA13038, 13036, 13716, } \\
13034 \text { and 13046) } \\
\text { Declaration of interest: Not reported. }\end{array}$ \\
\hline
\end{tabular}

Risk of bias

Bias

Random sequence generation (selection Low risk bias)
Authors' judgement Support for judgement

"Following baseline assessment, participants were randomised to condition using an urn randomisation. The urn program wasa program was used to balance participants within sites on gender, ethnicity, primary substance used, employment status, and whether the participant was mandated to treatment"

Method of allocation concealment not reported.

Urine toxicology.

Blinding (performance bias and detection Unclear risk bias)

All outcomes

Blinding of participants and personnel High risk (performance bias)

No blinding or incomplete blinding, and the outcome is likely to be influenced by lack of blinding

All outcomes

Blinding of outcome assessment (detection Low risk bias)

Urine toxicology was collected at all research assessment sessions (baseline, 28-day and 84-day follow-up)

All outcomes 


\section{Carroll 2006 (Continued)}

\begin{tabular}{l}
$\begin{array}{l}\text { Blinding of outcome assessor (detection } \\
\text { bias) subjective outcomes }\end{array}$ \\
\hline
\end{tabular}

\begin{tabular}{l|l|l}
\hline $\begin{array}{l}\text { Incomplete outcome data (attrition bias) } \\
\text { All outcomes }\end{array}$ & Low risk & ITT analysis performed. \\
\hline Selective reporting (reporting bias) & Low risk & $\begin{array}{l}\text { The study protocol is not available, but the published reports } \\
\text { include all expected outcomes, including those that were pre- } \\
\text { specified in the methods section }\end{array}$ \\
\hline Elliott $\mathbf{2 0 0 5}$ & RCT. & \\
\hline Methods &
\end{tabular}

Participants

53 Illicit drug users attending a drug clinic for methadone maintenance in Scotland. Mean age 30.6 years $(\mathrm{SD}=6.5)$, $53 \%$ male

$\mathrm{N}=24$ patients in intervention group $1 . \mathrm{N}=29$ patients in intervention group 2

Interventions

The psychological interventions that are tested in the present study were developed from CBT designed for those suffering from panic disorder or co-morbid anxiety who are withdrawing from BZDs. The following elements were used in the interventions: a) providing information and education about the effects of withdrawal, anxiety and sleep problems; b) visualising withdrawal symptoms; c) diaphragmatic breathing, progressive muscle relaxation exercises and guided imagery to address anxiety; d) sleep planning and encouraging good sleeping habits. Patients were requested to undertake fortnightly visits during which their diazepam medication was reviewed and the additional psychological support offered. Both the enhanced and limited intervention groups were given an initial orientation session, which focused on a general overview of the diazepam reduction plan and the psychological support. It outlined the frequency and details of reductions and familiarised the patient with the contents of the reduction handbook, which they were allowed to keep. The reduction handbook contained the information and descriptions of exercises designed to address three areas of difficulty that might be experienced when withdrawing from diazepam; withdrawal effects, anxiety and stress, and sleep difficulties. Both intervention groups undertook a further six visits during which they developed their skills and practised the basic exercises

Intervention group 1: Enhanced intervention - skills training and skills reinforcement. The enhanced group undertook further skills training whilst those in the limited intervention group were given verbal advice on request and referred back to the reduction handbook. The skills training involved further practice and development of the basic techniques designed to help with drug withdrawal, anxiety and sleep problems such as visualising withdrawal symptoms, breathing and relaxation exercises, and sleep planning Intervention group 2: Limited intervention - skills training and verbal advice on request

Outcomes

Outcome measures at baseline and at 6 months follow-up consisted of daily prescribed diazepam dose. Self-reported illicit drug use. Severity of dependence. Depression and sleep quality were also measured

Psychosocial interventions for benzodiazepine harmful use, abuse or dependence (Review) 
Elliott 2005 (Continued)

\begin{tabular}{ll}
\hline Notes & $\begin{array}{l}\text { Funding source: Chief Scientist Office, Scottish Executive UK } \\
\text { Declaration of interest: Not reported. }\end{array}$ \\
\hline
\end{tabular}

Risk of bias

Bias Authors' judgement Support for judgement

Random sequence generation (selection Low risk bias)

"After this block randomisation patients were randomly allocated to either arm by a statistician offsite and allocation was telephoned back to clinic. Patients were interviewed and then block randomised depending on whether dose of BDZ above or below equivalent of $30 \mathrm{mgs}$ diazepam then randomised"

Allocation concealment (selection bias) Low risk

"Patients were randomly allocated to either arm by a statistician offsite and allocation was telephoned back to clinic"

Blinding (performance bias and detection Low risk Prescription data for BZD dose.

bias)

All outcomes

Blinding of participants and personnel High risk (performance bias)

No blinding or incomplete blinding, and the outcome is likely All outcomes

to be influenced by lack of blinding

\begin{tabular}{|c|c|c|}
\hline $\begin{array}{l}\text { Blinding of outcome assessment (detection } \\
\text { bias) } \\
\text { All outcomes }\end{array}$ & Low risk & Prescription data for BZD dose. \\
\hline $\begin{array}{l}\text { Blinding of outcome assessor (detection } \\
\text { bias) subjective outcomes }\end{array}$ & Low risk & $\begin{array}{l}\text { "The interviewers were blind as to the allocation of the respon- } \\
\text { dent's intervention group" }\end{array}$ \\
\hline $\begin{array}{l}\text { Incomplete outcome data (attrition bias) } \\
\text { All outcomes }\end{array}$ & Low risk & ITT analyses performed. \\
\hline Selective reporting (reporting bias) & Low risk & $\begin{array}{l}\text { The study protocol is not available but the published reports } \\
\text { include all expected outcomes, including those that were pre- } \\
\text { specified in the method section }\end{array}$ \\
\hline
\end{tabular}

Elsesser 1996

\begin{tabular}{ll}
\hline Methods & RCT. \\
\hline Participants & 19 chronic BZD users recruited by newspaper advertising in Germany. At least 3 months \\
& $\begin{array}{l}5 \text { use of BZDs. One or more failed detox attempt. Mean use period } 12.17 \text { yrs (range } 0 . \\
5 \text { to } 30 \text { yrs). No current abuse of substances other than BZDs } \\
\text { N }=9 \text { patients in intervention group } 1 \text { ( } 5 \text { male; } 4 \text { female). } \mathrm{N}=10 \text { patients in control } \\
\text { group } 2 \text { (7 male; } 3 \text { female) }\end{array}$
\end{tabular}



treatment would be free. They were then given information about benzodiazepines and their effects, the danger of addiction with long-term use and a full account of withdrawal symptoms. Patients were also shown graphs of the course of withdrawal symptoms after abstinence illustrating their transient nature. It was stressed that patients were expected to complete the full treatment programme if they decided to take part. They were then given a diary form and asked to note their daily BZ intake and that of any other medication, their urge to take BZ, and the four most distressing symptoms they had experienced. They were also asked to note whether they had carried out relaxation exercises. The remainder of the session was devoted to breathing and relaxation exercises. Slow, abdominal breathing with short pauses at the beginning and end of each respiratory cycle was modelled and also carried out by the patients. Participant modelling of progressive relaxation was similarly carried out starting with the arm, neck and shoulder muscles. In Session 2, the full relaxation programme was carried out extending to the legs, the abdomen and face. During a final, deep relaxation phase, cue words such as 'relaxed' and 'warm' were introduced to be associated with that state. Patients were given cassette tapes and asked to practise relaxation twice daily. They were then instructed that, once learnt, the relaxation response could be used to counteract anxiety and discomfort. Early signs of discomfort were then explored and patients were asked to note down bodily changes or anxious thoughts that might occur at the onset of anxiety states. In Session 3, breathing and relaxation exercises were repeated once more. Thereafter and in the following sessions, the four symptoms that had been most frequently indicated in the weekly diary as having been distressing were dealt with. The most frequently named symptoms and their respective management technique are shown in Table 2 . Anxiety states were treated by means of anxiety management training, restlessness with advice as to distraction or physical exercise, and tachycardia with the Valsalva manoeuvre which was trained with a beat-by-beat pulse monitor, etc. Wherever applicable, techniques were carried out during the sessions until the patients mastered them. Stressful situations, symptoms or states were then imagined and counteracted with the newly learnt techniques during the treatment sessions. They were then to be used during the week between sessions with a subsequent discussion of successes and failures"

Intervention group 2: Anxiety management training. The first two sessions were identical to those of the CMT. During the remaining sessions, patients were asked to imagine unpleasant events or states which they had experienced, concentrate on early signs of distress and counteract them with relaxation. They were also given homework tasks and asked to apply their newly trained skill during the week between sessions with subsequent feedback Also measures for anxiety, depression, inventory of complaints, withdrawal symptoms and locus of control. Measures took place at baseline, every 2 weeks during treatment, final treatment and 6 month follow-up

\section{Risk of bias}

Psychosocial interventions for benzodiazepine harmful use, abuse or dependence (Review) 


\section{Elsesser 1996 (Continued)}

\begin{tabular}{|c|c|c|}
\hline Bias & Authors' judgement & Support for judgement \\
\hline $\begin{array}{l}\text { Random sequence generation (selection } \\
\text { bias) }\end{array}$ & Unclear risk & Method of randomisation not reported. \\
\hline Allocation concealment (selection bias) & Unclear risk & Method of allocation concealment not reported. \\
\hline $\begin{array}{l}\text { Blinding (performance bias and detection } \\
\text { bias) } \\
\text { All outcomes }\end{array}$ & Unclear risk & No objective measures were used. \\
\hline $\begin{array}{l}\text { Blinding of participants and personnel } \\
\text { (performance bias) } \\
\text { All outcomes }\end{array}$ & High risk & $\begin{array}{l}\text { No blinding or incomplete blinding, and the outcome is likely } \\
\text { to be influenced by lack of blinding }\end{array}$ \\
\hline $\begin{array}{l}\text { Blinding of outcome assessment (detection } \\
\text { bias) } \\
\text { All outcomes }\end{array}$ & Unclear risk & No objective measures were used. \\
\hline $\begin{array}{l}\text { Blinding of outcome assessor (detection } \\
\text { bias) subjective outcomes }\end{array}$ & Unclear risk & Insufficient information to permit judgement of low or high risk \\
\hline $\begin{array}{l}\text { Incomplete outcome data (attrition bias) } \\
\text { All outcomes }\end{array}$ & High risk & $\begin{array}{l}\text { Authors report high attrition with } 8 \text { patients leaving after first } \\
\text { treatment and } 17 \text { more after subsequent treatment sessions. A } \\
\text { total of } 25 \text { of } 44 \text { patients lost to follow-up. No ITT analyses } \\
\text { performed }\end{array}$ \\
\hline Selective reporting (reporting bias) & Low risk & $\begin{array}{l}\text { The study protocol is not available but the published reports } \\
\text { include all expected outcomes, including those that were pre- } \\
\text { specified in the method section }\end{array}$ \\
\hline
\end{tabular}

Gilbert 1993

\begin{tabular}{ll}
\hline Methods & RCT. \\
\hline Participants & $\begin{array}{l}60 \text { residents of two aged care facilities in Australia. } \\
\mathrm{N}=27 \text { patients in intervention group; } \mathrm{N}=33 \text { patients in control group }\end{array}$ \\
\hline Interventions & $\begin{array}{l}\text { Intervention group: Relaxation training and sleep hygiene. Relaxation training consisted } \\
\text { of eight } 40 \text { minute sessions over three weeks. A passive relaxation technique was used. } \\
\text { Participants were given a recording of relaxation training to practice between sessions. } \\
\text { Participants were given information about sleep, anxiety and medication use. They were } \\
\text { encouraged to use the relaxation procedures as a means of controlling anxiety and helping } \\
\text { with sleep; they were also encouraged to reduce their use of BZDs. Medical and support } \\
\text { staff were alerted to be vigilant to withdrawal effects and instructed to offer support } \\
\text { Control group: TAU. }\end{array}$ \\
\hline
\end{tabular}




\section{Gilbert 1993 (Continued)}

$\begin{array}{ll}\text { Outcomes } & \begin{array}{l}\text { Prescribed dose of BZDs. Sleep satisfaction, cognitive functioning, health rating and } \\ \text { mood rating. Measures were taken at baseline, } 1 \text { month and } 3 \text { months }\end{array}\end{array}$

mood rating. Measures were taken at baseline, 1 month and 3 months

Notes

Author supplied disaggregated data for self-reported measures

Funding source: South Australian Health Commission (Section 16 grant)

Declaration of interest: Not reported.

\section{Risk of bias}

\section{Bias}

Random sequence generation (selection Low risk bias)

Allocation concealment (selection bias) Unclear risk

Blinding (performance bias and detection Low risk bias)

All outcomes

Blinding of participants and personnel High risk (performance bias)

All outcomes

Blinding of outcome assessment (detection Low risk bias)

All outcomes

Blinding of outcome assessor (detection Unclear risk bias) subjective outcomes

Incomplete outcome data (attrition bias) Low risk All outcomes

Selective reporting (reporting bias)

Low risk

RCT.

\section{Gosselin 2006}

\begin{tabular}{ll}
\hline Methods & RCT. \\
\hline Participants & $\begin{array}{l}\text { Individuals experiencing generalised anxiety disorder who had used BZDs for at least } 4 \\
\text { days a week over the last } 12 \text { months in Canada. } \mathrm{N}=31 \text { patients in intervention group; } \\
\mathrm{N}=30 \text { patients in control group }\end{array}$
\end{tabular}

The study protocol is not available but the published reports include all expected outcomes, including those that were prespecified in the method section 


\section{Gosselin 2006 (Continued)}

\begin{tabular}{ll}
\hline Interventions & $\begin{array}{l}\text { Intervention group: CBT, plus tapering. } 12 \text { weeks of } 90 \text { minute sessions including psy- } \\
\text { chological education, cognitive restructuring, problem solving, cognitive exposure to } \\
\text { worries, situational exposure and relapse prevention } \\
\text { Control group: Non-specific treatment, plus tapering. } 12 \text { weeks of } 90 \text { minute sessions } \\
\text { including exploring life experiences to facilitate self-awareness and understanding of their } \\
\text { anxiety }\end{array}$ \\
\hline Outcomes & $\begin{array}{l}\text { Self-reported BZD use was measured each day during baseline assessment, intervention, } \\
2 \text { weeks post treatment and the } 2 \text { weeks prior to each follow-up assessment at 3, } 6 \text { and } \\
12 \text { months follow-up } \\
\text { Urinanlyses of weekly BZD dose (diazepam equivalent, mg) on patients reporting BZD } \\
\text { abstinence } \\
\text { Measurement of psychological symptoms (i.e. anxiety and depression). Participants mo- } \\
\text { tivation was measured pre- and post-treatment, as well as 3, } 6 \text { and } 12 \text { months follow-up }\end{array}$ \\
\hline Notes & $\begin{array}{l}\text { Funding source: The Canadian Institutes of Health Research and the Fonds de la } \\
\text { Recherche en Santé du Québec-Conseil Consultatif en Pharmacologie } \\
\text { Declaration of interest: Not reported. }\end{array}$ \\
\hline
\end{tabular}

\section{Risk of bias}

Bias

Authors' judgement Support for judgement

Random sequence generation (selection Low risk bias)

Block design with paired patients randomised with similar pretreatment scores on diazepam equivalent dose and length of time taking BZDs. Authors describe the matching and the randomisation having been done by an independent research associate

Allocation concealment (selection bias) Low risk "The matching and randomisation procedures were organised and administered by an independent research associate"

Blinding (performance bias and detection Low risk bias)

One primary outcome was objective that is BZD cessation. This All outcomes was based on urinalysis which was done on those reporting abstinence

Blinding of participants and personnel High risk (performance bias)

"Therapists met approximately the same number of patients in each condition. This procedure was chosen as it is difficult to

All outcomes keep the therapists blind to the treatment condition"

Blinding of outcome assessment (detection Low risk bias)

All outcomes

One primary outcome was objective that is BZD cessation, this was based on urinalysis which was only done on those reporting abstinence

Blinding of outcome assessor (detection High risk bias) subjective outcomes

"Therapists met approximately the same number of patients in each condition. This procedure was chosen as it is difficult to keep the therapists blind to the treatment condition" 


\section{Gosselin 2006 (Continued)}

\begin{tabular}{|c|c|c|}
\hline $\begin{array}{l}\text { Incomplete outcome data (attrition bias) } \\
\text { All outcomes }\end{array}$ & Low risk & $\begin{array}{l}\text { "All the analyses were conducted with an intention to treat ap- } \\
\text { proach" }\end{array}$ \\
\hline Selective reporting (reporting bias) & Low risk & $\begin{array}{l}\text { The study protocol is not available but the published report } \\
\text { includes the expected outcome which was pre-specified in the } \\
\text { methods section }\end{array}$ \\
\hline
\end{tabular}

Heather 2004

\begin{tabular}{ll}
\hline Methods & RCT. \\
\hline Participants & $\begin{array}{l}272 \text { long term }(>6 \text { months) BZD users attending general practice in England. } \mathrm{N}= \\
95 \text { patients in intervention group } 1 ; \mathrm{N}=88 \text { patients in intervention group 2; } \mathrm{N}=89 \\
\text { patients in control group }\end{array}$ \\
\hline Interventions & $\begin{array}{l}\text { Intervention group 1: Consultation group. Patients invited to a consultation with their } \\
\text { GP for a medication review } \\
\text { Intervention group 2: Discontinuation letter. Patients received a letter from their GP, } \\
\text { advising self-administered taper } \\
\text { Control group: Usual care. }\end{array}$ \\
\hline
\end{tabular}

Outcomes

Notes
Change in BZD intake as per prescription of BZDs. A 'true reducer' was somebody who had decreased intake by more than a quarter, including those who had stopped taking completely. Measures were taken at baseline and 6 month follow-up

Review group took the decision to combine consultation group with letter group versus control group

Funding source: Northern and Yorkshire Regional Health Authority R\&D Programme (ref: PCC16, January 1997)

Declaration of interest: Not reported.

\section{Risk of bias}

\begin{tabular}{|c|c|c|}
\hline Bias & Authors' judgement & Support for judgement \\
\hline $\begin{array}{l}\text { Random sequence generation (selection } \\
\text { bias) }\end{array}$ & Unclear risk & Method of randomisation not reported. \\
\hline Allocation concealment (selection bias) & Low risk & $\begin{array}{l}\text { "Patients returning an assessment questionnaire and consent } \\
\text { form were randomly allocated to one of three groups" } \\
\text { This was done independently of the doctors carrying out the } \\
\text { intervention }\end{array}$ \\
\hline $\begin{array}{l}\text { Blinding (performance bias and detection } \\
\text { bias) } \\
\text { All outcomes }\end{array}$ & High risk & $\begin{array}{l}\text { "Before the trial began, the researcher met participating GPs to } \\
\text { give guidance on how the consultation should be carried out" } \\
\text { This interaction in one of the three arms of the study could have } \\
\text { been a source of bias }\end{array}$ \\
\hline
\end{tabular}




\section{Heather 2004 (Continued)}

\begin{tabular}{|c|c|c|}
\hline $\begin{array}{l}\text { Blinding of participants and personnel } \\
\text { (performance bias) } \\
\text { All outcomes }\end{array}$ & Unclear risk & No subjective measures used. \\
\hline $\begin{array}{l}\text { Blinding of outcome assessment (detection } \\
\text { bias) } \\
\text { All outcomes }\end{array}$ & Low risk & $\begin{array}{l}\text { Information relating to BZD intake was prescribing data from } \\
\text { charts }\end{array}$ \\
\hline $\begin{array}{l}\text { Blinding of outcome assessor (detection } \\
\text { bias) subjective outcomes }\end{array}$ & Unclear risk & No subjective measures used. \\
\hline $\begin{array}{l}\text { Incomplete outcome data (attrition bias) } \\
\text { All outcomes }\end{array}$ & Low risk & No missing data. \\
\hline Selective reporting (reporting bias) & Low risk & $\begin{array}{l}\text { The study protocol is not available but the published report } \\
\text { includes the expected outcome which was pre-specified in the } \\
\text { methods section }\end{array}$ \\
\hline
\end{tabular}

King 2009

\begin{tabular}{ll}
\hline Methods & RCT. \\
\hline Participants & $\begin{array}{l}37 \text { patients attending an out-patient addiction treatment programme in USA. } 20 \text { ran- } \\
\text { domised to intervention (mean age }=42.7 \text { years; } 65 \% \text { female), } 17 \text { randomised to control } \\
\text { (mean age } 41.4 ; 47 \% \text { female) }\end{array}$ \\
\hline Interventions & $\begin{array}{l}\text { Intervention group: Internet-based counselling. This was specifically developed to deliver } \\
\text { verbal and visual based therapy to people with substance use problems. The same manual- } \\
\text { guided relapse control therapy group was used in both treatment conditions and is based } \\
\text { on exposure and training to several recovery-oriented skills (e.g. awareness and avoidance } \\
\text { of triggers; warning signs; drug refusal } \\
\text { Control group: Standard care. Participants assigned to this condition were scheduled to } \\
\text { attend on-site group counselling within the addiction services }\end{array}$ \\
\hline Outcomes & $\begin{array}{l}\text { Attendance at counselling sessions; urine toxicology; and treatment satisfaction. Measures } \\
\text { were taken at baseline and 6-week follow-up }\end{array}$ \\
\hline Notes & $\begin{array}{l}\text { Disaggregated BZD data provided by the author. } \\
\text { Funding source: Partial support from CRC-Health Group and Institutes for Behavior } \\
\text { Resources, Inc } \\
\text { Declaration of interest: Not reported. }\end{array}$ \\
\hline
\end{tabular}

\section{Risk of bias}

Bias

Random sequence generation (selection Unclear risk bias)

\section{Authors' judgement Support for judgement}

Method of randomisation not reported. 
King 2009 (Continued)

\begin{tabular}{|c|c|c|}
\hline Allocation concealment (selection bias) & Unclear risk & Method of allocation concealment not reported. \\
\hline $\begin{array}{l}\text { Blinding (performance bias and detection } \\
\text { bias) } \\
\text { All outcomes }\end{array}$ & Unclear risk & Urine toxicology. \\
\hline $\begin{array}{l}\text { Blinding of participants and personnel } \\
\text { (performance bias) } \\
\text { All outcomes }\end{array}$ & High risk & $\begin{array}{l}\text { No blinding or incomplete blinding, and the outcome is likely } \\
\text { to be influenced by lack of blinding }\end{array}$ \\
\hline $\begin{array}{l}\text { Blinding of outcome assessment (detection } \\
\text { bias) } \\
\text { All outcomes }\end{array}$ & Low risk & Urine toxicology. \\
\hline $\begin{array}{l}\text { Blinding of outcome assessor (detection } \\
\text { bias) subjective outcomes }\end{array}$ & Unclear risk & Insufficient information to permit judgment of low or high risk \\
\hline $\begin{array}{l}\text { Incomplete outcome data (attrition bias) } \\
\text { All outcomes }\end{array}$ & Low risk & No missing outcome data. \\
\hline Selective reporting (reporting bias) & Low risk & $\begin{array}{l}\text { The study protocol is not available but the published reports } \\
\text { include all expected outcomes, including those that were pre- } \\
\text { specified in the method section }\end{array}$ \\
\hline
\end{tabular}

Morin 2004

\begin{tabular}{l|l}
\hline Methods & RCT. \\
\hline Participants & $\begin{array}{l}76 \text { chronic users of BZDs for insomnia who wished to discontinue who were recruited } \\
\text { through newspaper advertising and physician referral in Canada. } 24 \text { participants in } \\
\text { intervention group 1; } 27 \text { participants in intervention group 2; 25 participants in the } \\
\text { control condition }\end{array}$ \\
\hline Interventions & $\begin{array}{l}\text { Intervention group 1: CBT only. This included weekly } 90 \text { min sessions in groups of } 4 \text { to } \\
6 \text { structured with education, cognitive and behavioural targeting a) facets of insomnia } \\
\text { including sleep restriction and b) stimulus control procedures }\end{array}$ \\
$\begin{array}{l}\text { Intervention group 2: CBT, plus taper. The combined CBT and tapering schedule } \\
\text { Control group: Taper only. Indvidualised step by step withdrawal schedule to stop BZDs } \\
\text { over } 10 \text { weeks. This included setting goals, stabilization on single BZD, reduction of } \\
25 \% \text { of initial dose every 2/52, introduction of increasing number of drug free nights, } \\
\text { schedule or hypnotic use not "as needed", and weekly sessions (15 to 20 minutes) with } \\
\text { prescribing doctor }\end{array}$ \\
\hline
\end{tabular}

Outcomes

Objective measures (blood and urine samples) and self-reported discontinuation or reduction of BZD use plus sleep quality and ratings for anxiety and depression. Measures were taken pre-treatment, post treatment, 3 months and 12 months

Psychosocial interventions for benzodiazepine harmful use, abuse or dependence (Review) 
Morin 2004 (Continued)

\begin{tabular}{ll}
\hline Notes & Follow-up paper Morin 2005. \\
& Review group took the decision to look at CBT plus taper versus taper only. This gives \\
a total of 52 participants \\
Funding source: NIH grant MH-55469. \\
Declaration of interest: Not reported. \\
\hline
\end{tabular}

Risk of bias

Bias

Random sequence generation (selection Low risk bias)

\begin{tabular}{|c|c|c|}
\hline Allocation concealment (selection bias) & Low risk & $\begin{array}{l}\text { Quote from an email with main author of study: } \\
\text { "Random numbers generated by computer and there was con- } \\
\text { cealment (for PI) of participants assignment for the study". } \\
\text { Above is not a quote from the paper but in an email from the PI }\end{array}$ \\
\hline $\begin{array}{l}\text { Blinding (performance bias and detection } \\
\text { bias) } \\
\text { All outcomes }\end{array}$ & Low risk & $\begin{array}{l}\text { The primary outcome is drug free status. This was confirmed } \\
\text { by blood and urine sampling }\end{array}$ \\
\hline $\begin{array}{l}\text { Blinding of participants and personnel } \\
\text { (performance bias) } \\
\text { All outcomes }\end{array}$ & High risk & No blinding and subjective outcomes subject to bias. \\
\hline $\begin{array}{l}\text { Blinding of outcome assessment (detection } \\
\text { bias) } \\
\text { All outcomes }\end{array}$ & Low risk & $\begin{array}{l}\text { The primary outcome is drug free status. This was confirmed } \\
\text { by blood and urine sampling }\end{array}$ \\
\hline $\begin{array}{l}\text { Blinding of outcome assessor (detection } \\
\text { bias) subjective outcomes }\end{array}$ & High risk & No blinding and subjective outcomes subject to bias. \\
\hline $\begin{array}{l}\text { Incomplete outcome data (attrition bias) } \\
\text { All outcomes }\end{array}$ & Low risk & "Data were analysed within an intent to treat framework". \\
\hline Selective reporting (reporting bias) & Low risk & $\begin{array}{l}\text { The study protocol is not available but the published report } \\
\text { includes the expected outcome which was pre-specified in the } \\
\text { methods section }\end{array}$ \\
\hline
\end{tabular}

Quote from an email with main author of study: "Random numbers generated by computer and use of sealed envelopes opened by research study coordinator when subjects ready for randomisation"

Authors' judgement Support for judgement 
Nathan 1986

\begin{tabular}{l|l}
\hline Methods & RCT. \\
\hline Participants & $\begin{array}{l}7 \text { females aged between } 25 \text { and } 50 \text { with a DSM-III diagnosis of generalised anxiety } \\
\text { disorder, with daily BZD use of over } 6 \text { months in USA. } 3 \text { participants in intervention } \\
\text { group } 1 ; 4 \text { participants in intervention group } 2\end{array}$ \\
\hline Interventions & $\begin{array}{l}\text { Intervention group 1: Intensive psychotherapy. Seen individually for 10-minute sessions } \\
\text { to simulate counselling and encouragement of traditional medical care. Individual psy- } \\
\text { choanalytical psychotherapy was offered, conducted for } 10 \text { weekly one hour sessions by } \\
\text { two individuals; a board certified psychiatrist and a licensed psychiatric social worker } \\
\text { Intervention group 2: Bio feedback assisted stress management. Seen individually for } \\
10 \text { weekly sessions by one of two licensed psychologists. Taped relaxation therapy twice } \\
\text { daily at home. EMG and skin temperature biofeedback in the office. GSR-II at home. } \\
\text { Limited supportive stress management counselling }\end{array}$ \\
\hline
\end{tabular}

Outcomes

Urine drug screens, Saliva drug screens, self-reported reduction in BZD use, measure of anxiety. Measures were taken at baseline, 1 month, 3 months and 12 months

Notes

Funding source: Not reported.

Declaration of interest: Not reported.

\section{Risk of bias}

\begin{tabular}{l|l|l}
\hline Bias & Authors' judgement & Support for judgement \\
\hline $\begin{array}{l}\text { Random sequence generation (selection } \\
\text { bias) }\end{array}$ & Unclear risk & Method of randomisation not reported. \\
\hline $\begin{array}{l}\text { Allocation concealment (selection bias) } \\
\begin{array}{l}\text { Blinding (performance bias and detection } \\
\text { bias) } \\
\text { All outcomes }\end{array}\end{array}$ & Low risk & Method of allocation concealment not reported. \\
\hline
\end{tabular}

Blinding of participants and personnel Unclear risk Subjective measures were not used.

(performance bias)

All outcomes

Blinding of outcome assessment (detection Low risk bias)

All outcomes

Blinding was not used. However, the objective outcomes were measured using urinalysis and saliva drug screens so this is un-

All outcomes

Blinding of outcome assessor (detection Unclear risk bias) subjective outcomes

likely to have been biased

\begin{tabular}{|c|c|c|}
\hline $\begin{array}{l}\text { Incomplete outcome data (attrition bias) } \\
\text { All outcomes }\end{array}$ & High risk & $\begin{array}{l}\text { "Of the seven patients who received treatment, only the four } \\
\text { in stress management treatment were available for 1-year follow } \\
\text { up" }\end{array}$ \\
\hline
\end{tabular}


Nathan 1986 (Continued)

Selective reporting (reporting bias) Unclear risk $\quad$ Outcomes not specified in methods and not clearly described.

O’Connor 2008

Methods $\quad$ Randomised controlled clinical trial.

Participants

Randomised participants were aged between 21 to 64 and were recruited through media announcements, clinic publicity and referrals. Initially 61 potential participants were reduced to 41 and these 41 were all assigned to control in this Canadian study. One year later a further 69 potential participants were reduced to 48 and these were randomly assigned to group support $(\mathrm{N}=24)$ or CBT plus group support $(\mathrm{N}=24)$. Three participants dropped out prior to baseline and were not replaced. Only data relating to the randomised participants are used within this review. Therefore, the total number of participants in the trial was $N=45$, with $N=23$ intervention 1 and $N=22$ for intervention 2

Interventions

Intervention group 1: CBT, plus taper. CBT was administered in manualised form over 20 weeks. The therapy aimed to enhance self-efficacy principally through normalizing expectations of withdrawal and attributions of withdrawal through boosting confidence in a) coping without BZD, b)coping with anxious inhibiting situations and through developing a belief in capacity to function autonomously from BZDs. Phase one was preparation ( 4 weeks), phase two was severance (16 weeks) and phase three was maintaining abstinence (duration unclear)

Intervention group 2: Group support, plus taper. Group programme comprised weekly meetings where exchanges took the form of open-ended discussions on themes such as 'What is anxiety?'. No direct actions or strategy to deal with problems was suggested. Participants reflected on discussions and themes throughout the week. Each week a different theme was discussed

Outcomes

The primary outcome measure was a successful taper at T1 which was further assessed by continuous success or failure 3 months post taper at T2. A completer was defined as a participant who completed the entire 20 week taper programme(T1). A succeeder (or responder) was defined as a participant who had ceased medication at 20 weeks. A relapse was defined as retaking medication at 3 months follow-up (T2). The criteria for success was total abstinence from BZDs and a further follow-up was performed at 7 to 15 months post T2 on those who had successfully tapered. A wide range of instruments was administered at the time points

Notes

Funding source: The Fonds de la Recherche en Santé du Québec and the Conseil Québécois de la Recherche Sociale (grant 961227)

Declaration of interest: Not reported.

Risk of bias

Bias

Authors' judgement

Support for judgement

Psychosocial interventions for benzodiazepine harmful use, abuse or dependence (Review)

Copyright () 2015 The Cochrane Collaboration. Published by John Wiley \& Sons, Ltd. 
O'Connor 2008 (Continued)

\begin{tabular}{|l|l|l|}
\hline $\begin{array}{l}\text { Random sequence generation (selection } \\
\text { bias) }\end{array}$ & $\begin{array}{l}\text { "After initial recruitment of } 130 \text { individuals } \\
\text { some were assigned sequentially but not ran- } \\
\text { domly to tapering or treatment as usual. Even- } \\
\text { tually, } 48 \text { were randomly assigned, to either a } \\
\text { group CBT or a non-directive group support } \\
\text { condition, on the basis of a random sequence } \\
\text { generator" }\end{array}$ \\
\hline
\end{tabular}

Allocation concealment (selection bias) Unclear risk $\quad$ Method of allocation concealment not reported.

Blinding (performance bias and detection Low risk bias)

"All treating physicians included in the study were blind as to membership of the referred par-

All outcomes ticipants"

Blinding of participants and personnel Low risk (performance bias)

All outcomes

"All treating physicians included in the study were blind as to membership of the referred participants"

Blinding of outcome assessment (detection Low risk bias)

Objective outcome was taper dose.Unlikely to

have been influenced

All outcomes

Blinding of outcome assessor (detection Unclear risk bias) subjective outcomes

Subjective measures were mainly a series of validated instruments and it is difficult to say from the paper whether the measures were influenced

Incomplete outcome data (attrition bias) Unclear risk All outcomes

"A limitation of the group comparisons was that questionnaire measures were not available on all participants at follow up"

Overall follow-up for the primary outcome measure(successful/non-successful taper) was $31 / 48$

Selective reporting (reporting bias) Low risk

The study protocol is not available but the published report includes the expected outcome which was pre-specified in the methods section

Otto 1993

Methods

Participants

\section{RCT.}

33 outpatients treated for panic disorder with alprazolam or clonazepam for a minimum of 6 months and seeking to discontinue this treatment were selected for the study in the USA. 17 participants in the intervention group; 16 participants in the control group

Intervention group: CBT, plus taper. Participants receiving taper plus CBT received all the elements of taper as usual, but also received 10 sessions of CBT in weekly group sessions of 90 minutes' duration for the first five sessions and 60 minutes for the last five sessions. The CBT included the following: identification of symptoms of both 
Otto 1993 (Continued)

withdrawal and panic; structured exposure to somatic sensations of anxiety and panic; teaching and practice of somatic coping skills

Control group: Taper as usual condition. Participants received information on discontinuation effects, a slow taper schedule, weekly clinical monitoring, and general encouragement and support regarding discontinuation difficulties

Outcomes

BZD use as measured by dose prescribed and panic attack frequency at baseline, post treatment and 3 months

Notes

Funding source: NIMH Faculty Scholar Award MH-19600 and a grant from Roche Laboratories, Hoffman LaRoche, Inc

Declaration of interest: Not reported.

\section{Risk of bias}

Bias

Random sequence generation (selection Low risk bias)

\begin{tabular}{lll} 
Allocation concealment (selection bias) & Unclear risk \\
\hline $\begin{array}{l}\text { Blinding (performance bias and detection } \\
\text { bias) }\end{array}$ & Unclear risk
\end{tabular}
bias)

All outcomes

Blinding of participants and personnel High risk (performance bias)

All outcomes
Authors' judgement Support for judgement

"Patients were block randomised to one of two treatment programs"

Method of allocation concealment not reported.

"The principal dependent measure was the proportion of patients successfully completing the scheduled taper"

While this measure's objectivity is normally ascribed to the fact that it is prescription based that is not stated explicitly in the text

"The differences between the groups at each evaluation point were analysed with Mann-Whitney U tests. These analyses were compromised by patients who failed to continue their medication taper and by missing values for some subjects who did not complete or return questionnaires"

Blinding of outcome assessment (detection Unclear risk bias)

All outcomes tients successfully completing the scheduled taper" While this measure's objectivity is normally ascribed to the fact that it is prescription based that is not stated explicitly in the text

"The differences between the groups at each evaluation point were analyzed with Mann-Whitney U tests. These analyses were compromised by patients who failed to continue their medication taper and by missing values for some subjects who did not complete or return questionnaires"
Incomplete outcome data (attrition bias) Low risk All outcomes
No missing data. 
Otto 1993 (Continued)

Selective reporting (reporting bias) Low risk

The study protocol is not available but the published report includes the expected outcome which was pre-specified in the methods section

Otto 2010

\begin{tabular}{ll} 
Methods & RCT. \\
\hline Participants & 47 participants were outpatients seeking treatment for help with BZD discontinuation \\
& in the USA. Individuals who contacted the clinic were screened by telephone for general \\
medical, diagnostic, and treatment eligibility and interest in research participation & 16 participants in intervention group $1 ; 16$ participants in intervention group 2; 15 \\
participants in the control group
\end{tabular}

Interventions

Intervention group 1: CBT plus taper. Participants receiving taper plus CBT received all of the elements of TAU, but also received eight weekly, individual exposure- based CBT sessions, followed by three booster sessions scheduled at intervals of two weeks, four weeks, and six weeks, respectively. Patients met independently with their TAU and CBT clinicians. Patients in the CBT initiated their TAU taper after the third CBT session. All sessions lasted $60 \mathrm{~min}$, except the initial 90-min session. The CBT combined four primary treatment components: an informational component, interoceptive exposure, somatic coping skills, and cognitive restructuring

Intervention group 2: Individual relaxation treatment (IRT), plus taper. In addition to the non-specific support provided by therapist contact, IRT involves two treatment components: an informational component and progressive muscle-relaxation training. The informational component includes a review of the time course and nature of withdrawal symptoms and discussion of these symptoms in an individual setting as they occur. Relaxation training includes training and review of progressive muscle-relaxation procedures in session and home assignment of these skills

Control group: Routine care - 'taper as usual' (TAU). Elements of this treatment included information on discontinuation effects, a slow-taper schedule, weekly clinical monitoring, and encouragement and support regarding discontinuation difficulties. The withdrawal schedule for all patients taking alprazolam was a reduction of the daily dose by $0.25 \mathrm{mg}$ every 2 days for doses above $2.0 \mathrm{mg}$. Patients who started at $2.0 \mathrm{mg}$ or below, or who reached this level during their taper, underwent a reduction of the daily dose by $0.125 \mathrm{mg}$ every 2 days. Accordingly, the taper lasted approximately 5 weeks for patients with a starting daily dose of $2 \mathrm{mg}$, 7 weeks for patients taking $4 \mathrm{mg}$, and 9 weeks for patients taking $6 \mathrm{mg}$ of alprazolam at baseline. Alprazolam was prescribed on a four-times-per-day (q.i.d.) basis, with the first morning dose being the last to be discontinued. Patients taking clonazepam followed a similar taper schedule adjusted for the approximate $2: 1$ difference in potency relative to alprazolam and the smallest pill size $(0.5 \mathrm{mg})$ available at the time for clonazepam. Hence, patients taking clonazepam had their daily dose reduced by $0.25 \mathrm{mg}$ every four days for daily doses above $1.0 \mathrm{mg}$, or by 0 . $25 \mathrm{mg}$ every eight days for daily doses of $1.0 \mathrm{mg}$ or less. Patients taking clonazepam were prescribed on a twice-per-day (b.i.d.) basis. Patients recorded the actual number of doses they took in the space provided on their written taper schedule, which was collected at each visit. This written withdrawal schedule served as a guide for dose reduction and was complemented by take-home panic diaries

Psychosocial interventions for benzodiazepine harmful use, abuse or dependence (Review)

Copyright @ 2015 The Cochrane Collaboration. Published by John Wiley \& Sons, Ltd. 


\section{Otto 2010 (Continued)}

\begin{tabular}{ll} 
Outcomes & $\begin{array}{l}\text { Self-reported BZD use. Psychological constructs of anxiety and depression and with- } \\
\text { drawal symptoms. Measures were reported at baseline, } 2 \text { weeks (post treatment), } 3 \\
\text { months and } 6 \text { months }\end{array}$ \\
\hline Notes & $\begin{array}{l}\text { Funding source: NIDA grant R10 DA09692. } \\
\text { Declaration of interest: "The authors are aware of no conflicts with the content of this } \\
\text { manuscript, nonetheless Dr. Otto would like to report current consultant and research } \\
\text { support from Schering-Plough, and royalties received in the last year for use of the SIGH- } \\
\text { A from Lilly. Dr. Pollack would like to report advisory board or consultation or both } \\
\text { from Brain Cells, Eli Lilly, Medavante, Mindsite, Targia Pharmaceuticals, and Pfizer; } \\
\text { research grant support from Bristol Myers Squibb, Forest Laboratories, Glaxo SmithK- } \\
\text { line, Eli Lilly, NCCAM, NIDA, NIMH, and Sepracor; CME supported activities from } \\
\text { Astra Zeneca, Sepracor, and Pfizer; equity interests in Medavante, Mensante Corpora- } \\
\text { tion, Mindsite, and Targia Pharmaceuticals; and royalty or patent payments regarding } \\
\text { the SIGH-A and SAFER interviews. Dr. Pollack would like to report advisory board or } \\
\text { consultation or both from Astra Zeneca, Cephalon, Forest Laboratories, Glaxo SmithK- } \\
\text { line, Janssen, Lilly, NARSAD, NIMH, Pfizer, UCB- Pharma, Sepracor; and speaking/ } \\
\text { CME supported activities from MGHPsychiatry Academy, Astra Zeneca, and Pfizer. Dr. } \\
\text { Worthington would like to report grant-research support from Eli Lilly \& Company, } \\
\text { Pfizer Inc, and Sepracor; and speaker support from Pfizer Inc. The remaining authors } \\
\text { have no conflicts to report" }\end{array}$ \\
\hline
\end{tabular}

Risk of bias

\begin{tabular}{|c|c|c|}
\hline Bias & Authors' judgement & Support for judgement \\
\hline $\begin{array}{l}\text { Random sequence generation (selection } \\
\text { bias) }\end{array}$ & Low risk & $\begin{array}{l}\text { "Patients with panic disorder were randomized (based on a ran- } \\
\text { domization table created for this study)" }\end{array}$ \\
\hline
\end{tabular}

\begin{tabular}{l|l|l}
\hline Allocation concealment (selection bias) & Unclear risk & Method of allocation concealment not reported. \\
\hline $\begin{array}{l}\text { Blinding (performance bias and detection } \\
\text { bias) } \\
\text { All outcomes }\end{array}$ & Low risk & Prescription records of dose of BZDs. \\
\hline
\end{tabular}

\begin{tabular}{l|l}
$\begin{array}{l}\text { Blinding of participants and personnel High risk } \\
\text { (performance bias) }\end{array}$ & $\begin{array}{l}\text { No blinding or incomplete blinding, and the outcome is likely } \\
\text { to be influenced by lack of blinding }\end{array}$
\end{tabular}

Blinding of outcome assessment (detection Low risk bias)

All outcomes

Blinding of outcome assessor (detection Low risk bias) subjective outcomes

\begin{abstract}
"Study assessments were conducted by monitoring physicians (who were blind to treatment condition) at baseline, post-medication discontinuation, and follow-up assessments at 2 weeks (post-treatment) and 3 and 6 months post-discontinuation"
\end{abstract}

"Study assessments were conducted by monitoring physicians (who were blind to treatment condition) at baseline, post-medication discontinuation, and follow-up assessments at 2 weeks (post-treatment) and 3 and 6 months post-discontinuation" 


\section{Otto 2010 (Continued)}

\begin{tabular}{|c|c|c|}
\hline $\begin{array}{l}\text { Incomplete outcome data (attrition bias) } \\
\text { All outcomes }\end{array}$ & Low risk & $\begin{array}{l}\text { "At three month follow-up, a number of patients missed evalu- } \\
\text { ation appointments. If patients had a BZ-free status at both the } \\
\text { previous visit (acute outcome visit) and the subsequent visit (6- } \\
\text { month visit), a BZ-free status was assigned; otherwise missing } \\
\text { values were assumed to be treatment failures, ensuring a conser- } \\
\text { vative analysis of discontinuation success rates" }\end{array}$ \\
\hline Selective reporting (reporting bias) & Low risk & $\begin{array}{l}\text { The study protocol is not available but the published reports } \\
\text { include all expected outcomes, including those that were pre- } \\
\text { specified in the method section }\end{array}$ \\
\hline \multicolumn{3}{|l|}{ Oude Voshaar 2003 a } \\
\hline Methods & RCT. & \\
\hline Participants & \multicolumn{2}{|c|}{$\begin{array}{l}180 \text { long term BZD users attending general practice in the Netherlands. } 73 \text { participants } \\
\text { in the intervention group 1; } 73 \text { participants intervention group 2; } 34 \text { participants in } \\
\text { control group }\end{array}$} \\
\hline Interventions & \multicolumn{2}{|c|}{$\begin{array}{l}\text { Intervention group 1: Group CBT plus taper. This included: } 1 \text {. psycho education con- } \\
\text { cerning the advantages and disadvantages of long-term BZD use; } 2 \text {. teaching and prac- } \\
\text { tising relaxation exercises by means of progressive relaxation cognitive restructuring of } \\
\text { the interpretation of withdrawal symptoms } \\
\text { Intervention group 2: Taper only. Participants who were not using diazepam were trans- } \\
\text { ferred to an equivalent dose of diazepam for } 2 \text { weeks by their own doctor. For partici- } \\
\text { pants taking more than one BZD, the dosages were added together. The daily dose of } \\
\text { diazepam was reduced by } 25 \% \text { a week during four weekly visits. Participants had the } \\
\text { choice to divide the last step into two steps of } 12.5 \% \text { for } 4 \text { days } \\
\text { Control group: Usual care. This group did not receive any help with BZD reduction }\end{array}$} \\
\hline Outcomes & \multicolumn{2}{|c|}{$\begin{array}{l}\text { Reduction in BZD use by self-report and verified by prescriptions. Psychological con- } \\
\text { structs such as mood and well being; and withdrawal symptoms. Cognitive memory skills } \\
\text { were also assessed. Assessment of outcomes occurred at baseline and } 3 \text { month follow-up }\end{array}$} \\
\hline Notes & \multicolumn{2}{|c|}{$\begin{array}{l}\text { Review group took the decision to look at intervention } 1 \text { ( } 73 \text { participants) versus inter- } \\
\text { vention } 2 \text { ( } 73 \text { participants) } \mathrm{N}=146 \\
\text { Follow-up paper: Oude Voshaar } 2006 \mathrm{~d} \text {. } \\
\text { Funding source: The Dutch Health Care Insurance Council. } \\
\text { Declarations of interest: None. }\end{array}$} \\
\hline
\end{tabular}

\section{Risk of bias}

\section{Bias}

Authors' judgement Support for judgement

Random sequence generation (selection Low risk bias)
Unclear risk

Method of allocation concealment not reported. 


\begin{tabular}{l|l|l}
$\begin{array}{l}\text { Blinding (performance bias and detection } \\
\text { bias) } \\
\text { All outcomes }\end{array}$ & Unclear risk & Prescription data for BZD use. \\
\hline $\begin{array}{l}\text { Blinding of participants and personnel } \\
\text { (performance bias) } \\
\text { All outcomes }\end{array}$ & High risk & $\begin{array}{l}\text { No blinding or incomplete blinding, and the outcome is likely } \\
\text { to be influenced by lack of blinding }\end{array}$ \\
\hline $\begin{array}{l}\text { Blinding of outcome assessment (detection } \\
\text { bias) } \\
\text { All outcomes }\end{array}$ & Low risk & Prescription data for BZD use. \\
\hline $\begin{array}{l}\text { Blinding of outcome assessor (detection } \\
\text { bias) subjective outcomes }\end{array}$ & Unclear risk & Insufficient information to permit judgement of low or high risk \\
\hline
\end{tabular}

Incomplete outcome data (attrition bias) Low risk ITT analysis performed.

All outcomes

Selective reporting (reporting bias) Low risk

The study protocol is not available but the published reports include all expected outcomes, including those that were prespecified in the method section

Parr 2013

\begin{tabular}{ll}
\hline Methods & RCT. \\
\hline Participants & $\begin{array}{l}6 \text { individuals attending their GP who had been prescribed BZDs for longer than } 3 \\
\text { months in Canada. 3 participants in the intervention group; 3 participants in the control } \\
\text { group }\end{array}$ \\
\hline Interventions & $\begin{array}{l}\text { Intervention group: Immediate mailed CBT plus taper. The content of the mailed CBT } \\
\text { package included making decisions; coping with withdrawal and after; sleeping better; } \\
\text { straight thinking; be active; finding a supporter; eating when you don't feel like it; coping } \\
\text { with worry; planning your day; keeping on track; life after 'benzos'; returning to benzo } \\
\text { use. It comprised 12 weekly newsletters, together with feedback on assessments and on } \\
\text { the progress of their dose reduction }\end{array}$ \\
\hline Control group: Delayed mailed CBT, plus taper. \\
\hline Outcomes & $\begin{array}{l}\text { Self-reported consumption of BZDs at baseline, and } 3 \text { months. This study had a waiting } \\
\text { list controlled design, whereby participants in the control group received the intervention } \\
\text { post } 3 \text { month follow-up. Therefore, both the } 6 \text { month and } 12 \text { month data collection } \\
\text { was not relevant for the current review }\end{array}$ \\
\hline Fotes & $\begin{array}{l}\text { Funding source: Not reported. } \\
\text { Declarations of interest: Not reported. }\end{array}$ \\
\hline
\end{tabular}

\section{Risk of bias}

Psychosocial interventions for benzodiazepine harmful use, abuse or dependence (Review)

Copyright @ 2015 The Cochrane Collaboration. Published by John Wiley \& Sons, Ltd. 
Parr 2013 (Continued)

\begin{tabular}{|c|c|c|}
\hline Bias & Authors' judgement & Support for judgement \\
\hline $\begin{array}{l}\text { Random sequence generation (selection } \\
\text { bias) }\end{array}$ & Low risk & $\begin{array}{l}\text { "Once participants were judged to be eligible to participate and } \\
\text { consented to the trial, they completed the baseline assessments } \\
\text { and were randomly allocated to receive M-CBT immediately or } \\
\text { after } 3 \text { months. The random allocation process was conducted } \\
\text { by an independent research associate and occurred in blocks of } \\
\text { six participants, using a series of random permutations of the } \\
\text { numbers 1-6 in order to ensure approximate equalisation across } \\
\text { groups" }\end{array}$ \\
\hline
\end{tabular}

Allocation concealment (selection bias) Low risk "Envelopes were provided to the research team in numbered order and allocated to participants when they commenced with the program"

Blinding (performance bias and detection Unclear risk No objective measure used.

bias)

All outcomes

Blinding of participants and personnel High risk (performance bias)

No blinding or incomplete blinding, and the outcome is likely All outcomes

Blinding of outcome assessment (detection Unclear risk bias) to be influenced by lack of blinding

All outcomes

Blinding of outcome assessor (detection Low risk bias) subjective outcomes

No objective measure used.

viewed them by telephone to confirm and clarify their consump-
vion
tion data"

Incomplete outcome data (attrition bias) Low risk All outcomes

Selective reporting (reporting bias)
Missing outcome data balanced in numbers across intervention groups, with similar reasons for missing data across groups

The study protocol is not available but the published reports include all expected outcomes, including those that were prespecified in the method section

\section{Scherbaum 2005}

\begin{tabular}{ll}
\hline Methods & RCT. \\
\hline Participants & $\begin{array}{l}73 \text { patients attending for their first episode of methadone maintenance treatment in } \\
\text { Germany } \\
41 \text { participants in the intervention group; } 32 \text { participants in the control group }\end{array}$
\end{tabular}


Scherbaum 2005 (Continued)

\begin{tabular}{|c|c|c|}
\hline Interventions & \multicolumn{2}{|c|}{$\begin{array}{l}\text { Intervention group: Cognitive behavioural group psychotherapy. } 20 \text { group psychother- } \\
\text { apy sessions, lasting } 90 \text { minutes each. The psychotherapy was aimed at the patient's } \\
\text { understanding of the individual situations pre-disposing them to drugs. Dysfunctional } \\
\text { cognition was identified and alternative cognition and behaviours established. Strategies } \\
\text { for relapse prevention were identified } \\
\text { Control group: Standard methadone maintenance. }\end{array}$} \\
\hline Outcomes & \multicolumn{2}{|c|}{$\begin{array}{l}\text { Drug use, as measured by } 5 \text { randomised urine screens per month, at onset of treatment, } \\
\text { end of treatment, } 3 \text { months and } 6 \text { months follow-up. Intensity of drug use was defined } \\
\text { as the relative frequency of urine samples positive for BZDs }\end{array}$} \\
\hline Notes & \multicolumn{2}{|c|}{$\begin{array}{l}\text { Funding source: Supported by the Deutsche Forschungsgemeinschaft (DFG): Ga-564/ } \\
2-1 \\
\text { Declaration of interest: Not reported. }\end{array}$} \\
\hline \multicolumn{3}{|l|}{ Risk of bias } \\
\hline Bias & Authors' judgement & Support for judgement \\
\hline $\begin{array}{l}\text { Random sequence generation (selection } \\
\text { bias) }\end{array}$ & Low risk & Randomised by flicking a coin. \\
\hline Allocation concealment (selection bias) & Unclear risk & Method of allocation concealment not reported. \\
\hline $\begin{array}{l}\text { Blinding (performance bias and detection } \\
\text { bias) } \\
\text { All outcomes }\end{array}$ & Low risk & $\begin{array}{l}\text { While the treatment providers were in regular contact with the } \\
\text { patients the method of ascertainment (regular urine screening) } \\
\text { of the objective outcome was not susceptible to bias }\end{array}$ \\
\hline $\begin{array}{l}\text { Blinding of participants and personnel } \\
\text { (performance bias) } \\
\text { All outcomes }\end{array}$ & Unclear risk & No subjective measures used. \\
\hline $\begin{array}{l}\text { Blinding of outcome assessment (detection } \\
\text { bias) } \\
\text { All outcomes }\end{array}$ & Low risk & $\begin{array}{l}\text { The main outcome criterion was the use of drugs as measured } \\
\text { by five randomised urine screens per month }\end{array}$ \\
\hline $\begin{array}{l}\text { Blinding of outcome assessor (detection } \\
\text { bias) subjective outcomes }\end{array}$ & Unclear risk & No subjective measures used. \\
\hline $\begin{array}{l}\text { Incomplete outcome data (attrition bias) } \\
\text { All outcomes }\end{array}$ & Low risk & No missing data. \\
\hline Selective reporting (reporting bias) & Low risk & $\begin{array}{l}\text { The study protocol is not available but the published report } \\
\text { includes the expected outcome which was pre-specified in the } \\
\text { methods section }\end{array}$ \\
\hline
\end{tabular}

Psychosocial interventions for benzodiazepine harmful use, abuse or dependence (Review) 
Spiegel 1994

\begin{tabular}{ll}
\hline Methods & RCT. \\
\hline Participants & $\begin{array}{l}\text { Patients attending an anxiety disorder clinic in the USA who experience panic attacks. } \\
21 \text { patients in total (11 intervention; } 10 \text { control group). Mean age } 38 \text { years; four men } \\
\text { and } 17 \text { women. All patients were stabilized on alprazolam before enrolment }\end{array}$ \\
\hline Interventions & $\begin{array}{l}\text { Intervention group: CBT plus taper. Treatment included: education about panic disor- } \\
\text { der; training in slow diaphragmatic breathing; cognitive restructuring; and interoceptive } \\
\text { exposure (i.e. exposure to feared bodily symptoms). During the stable dose and taper } \\
\text { phases all participants (both intervention and control) met weekly with a psychiatrist, } \\
\text { who was blind to group assignment, for supportive medical management. During the } \\
\text { follow-up after completing drug taper or leaving the taper protocol, subjects were seen } \\
\text { briefly at 2-week intervals for } 3 \text { months and then once again at } 6 \text { months for a final visit } \\
\text { Control group: Taper only. }\end{array}$
\end{tabular}

Outcomes

Number of patients who completed all taper steps and remained abstinent measured weekly for 6 months. Withdrawal symptoms. Side effects during stable dose phase. Drug substitution: Alcohol and nicotine use

Notes

Follow-up paper Bruce 1999 - long term follow-up data.

Funding source: Supported in part by NIH grant RR-05369 from the National Center for Research Resources and a grant from the Upjohn Company

Declaration of interest: Not reported.

\section{Risk of bias}

Bias

Random sequence generation (selection Unclear risk bias)

\section{Authors' judgement Support for judgement}

Method of randomisation not reported.

Method of allocation concealment not reported.

Prescription BZD dose.

Blinding (performance bias and detection Unclear risk bias)

All outcomes

Blinding of participants and personnel Low risk (performance bias)

No blinding or incomplete blinding, and the outcome is likely to be influenced by lack of blinding

All outcomes

Blinding of outcome assessment (detection Unclear risk bias)

All outcomes

"Extensive precautions were taken to preserve the blind status of the treating psychiatrist, which was tested at the end of the study"

Blinding of outcome assessor (detection Low risk bias) subjective outcomes
"One participant in the intervention group was dropped during the taper phase as she had been placed on a regimen of centrally acting medication for a medical condition unrelated to anxiety. She had been on schedule with taper at the time of termination. 
All other participants completed the study"

\begin{tabular}{|c|c|c|}
\hline $\begin{array}{l}\text { Incomplete outcome data (attrition bias) } \\
\text { All outcomes }\end{array}$ & Low risk & $\begin{array}{l}\text { One participant in the intervention group was dropped during } \\
\text { the taper phase as she had been placed on a regimen of centrally } \\
\text { acting medication for a medical condition unrelated to anxiety. } \\
\text { She had been on schedule with taper at the time of termination. } \\
\text { All other participants completed the study }\end{array}$ \\
\hline
\end{tabular}

Selective reporting (reporting bias) Low risk

The study protocol is not available but the published reports include all expected outcomes, including those that were prespecified in the method section

Ten Wolde 2008

\begin{tabular}{ll}
\hline Methods & RCT. \\
\hline Participants & 695 chronic BZD users attending attending their GP in the Netherlands \\
& 228 participants in intervention group $1 ; 256$ participants in intervention group 2 and \\
& 211 participants in control group \\
& After 12 months 187 participants were lost to follow-up, thus giving a total of 508 par- \\
ticipants: 163 participants in the intervention group $1 ; 186$ participants in intervention \\
group 2 and 159 participants in control group
\end{tabular}

Interventions

Intervention group 1: Single tailored letter. The single tailored letter intervention consisted of one letter of five to six pages of information (approximately 1200 words) in which all of three psychological determinants were addressed. The information was designed to: (i) increase the perceptions of the positive outcome expectations of discontinuing BZD use (e.g. it was argued that patients may function better cognitively and may evaluate themselves more positively); (ii) lower the perceptions of the positive outcome expectations of the use of BZDs (by explaining the development of tolerance and a possible placebo effect); and (iii) increase self-efficacy expectations with regard to discontinuing usage (by offering several skills to reach abstinence, such as making a plan to cut down BZD use and by offering alternatives in order to cope with worrying thoughts)

Intervention group 2: The multiple tailored letter intervention consisted of three letters of about three pages each (approximately 400 words), sent at intervals of 1 month. In the multiple tailored intervention, the first tailored letter was designed to increase the perceptions of the positive outcome expectations of discontinuing BZD usage and to lower the perceptions of the positive outcome expectations of the use of BZDs. The second tailored letter was designed to increase self-efficacy expectations with regard to discontinuing usage, while the content of the third letter provided more skills for discontinuing usage, or provided a summary of the information in the first two letters, depending on the individual needs detected in the third assessment. In addition, in the introduction of the second and third letters, participants were provided with progress feedback. Individual changes in BZD use were mentioned

Control group: Standard letter from GP outlining the disadvantages of BZD use and advising to quit use of BZDs. The letter consisted of approximately 200 words 
Ten Wolde 2008

Notes
Review group took the decision to examine intervention 1 (single letter) versus GP letter
163 participants in intervention group 1 and 159 participants in control group. Reported
figures are based on the 12 month data
Funding source: Dutch Council for Health Insurance.
Declaration of interest: Not reported.

Risk of bias

\begin{tabular}{|c|c|c|}
\hline Bias & Authors' judgement & Support for judgement \\
\hline $\begin{array}{l}\text { Random sequence generation (selection } \\
\text { bias) }\end{array}$ & Unclear risk & Method of randomisation not reported. \\
\hline Allocation concealment (selection bias) & High risk & $\begin{array}{l}\text { GPs could select out patients who had severe comorbidity or } \\
\text { psychological problems }\end{array}$ \\
\hline $\begin{array}{l}\text { Blinding (performance bias and detection } \\
\text { bias) } \\
\text { All outcomes }\end{array}$ & Unclear risk & No objective measures were used. \\
\hline $\begin{array}{l}\text { Blinding of participants and personnel } \\
\text { (performance bias) } \\
\text { All outcomes }\end{array}$ & High risk & $\begin{array}{l}\text { No blinding or incomplete blinding, and the outcome is likely } \\
\text { to be influenced by lack of blinding }\end{array}$ \\
\hline $\begin{array}{l}\text { Blinding of outcome assessment (detection } \\
\text { bias) } \\
\text { All outcomes }\end{array}$ & Unclear risk & No objective measures were used. \\
\hline $\begin{array}{l}\text { Blinding of outcome assessor (detection } \\
\text { bias) subjective outcomes }\end{array}$ & High risk & $\begin{array}{l}\text { No blinding of outcome assessment, and the outcome measure- } \\
\text { ment is likely to be influenced by lack of blinding }\end{array}$ \\
\hline $\begin{array}{l}\text { Incomplete outcome data (attrition bias) } \\
\text { All outcomes }\end{array}$ & Low risk & ITT analysis performed. \\
\hline Selective reporting (reporting bias) & Low risk & $\begin{array}{l}\text { The study protocol is not available but the published reports } \\
\text { include all expected outcomes, including those that were pre- } \\
\text { specified in the methods section }\end{array}$ \\
\hline Methods & \multicolumn{2}{|c|}{ Randomised controlled clinical trial. } \\
\hline Participants & \multicolumn{2}{|c|}{$\begin{array}{l}139 \text { patients aged } 15 \text { to } 74 \text { who were taking BZDs at least five times a week for over a } \\
\text { year attending a health centre in Spain } \\
73 \text { participants in intervention group and } 66 \text { participants in control group }\end{array}$} \\
\hline
\end{tabular}

Psychosocial interventions for benzodiazepine harmful use, abuse or dependence (Review) 
Vicens 2006 (Continued)

\begin{tabular}{ll}
\hline Interventions & $\begin{array}{l}\text { Intervention group: Advice, tapering and bi-weekly visits to a GP. The intervention } \\
\text { consisted of an interview with a doctor at first visit, with a standardised message. The } \\
\text { message had information on BZDs, side effects, problems of long-term use and how to } \\
\text { withdraw. Treatment of symptoms versus treatment of causes was discussed. At follow- } \\
\text { up visits possible withdrawal symptoms were discussed as well as positive reinforcement } \\
\text { of achievements. Patients in the intervention group underwent a gradual reduction of } \\
\text { BZD dose, with visits every } 15 \text { days. The dose was reduced between } 10 \text { and } 25 \% \text { of the } \\
\text { initial dose fortnightly } \\
\text { Control group: Standard care. Managed according to usual practice and informed of the } \\
\text { convenience of reducing the use of BZDs }\end{array}$ \\
\hline
\end{tabular}

Outcomes

Self-reported BZD use at 6 months, 12 months and in the related study, at 3 years. End points were: success, no use or no more than once every 15 days; reduced, at least a $50 \%$ reduction in initial dose; failure, no change or a decrease smaller than $50 \%$

Notes

Additional data supplied by author.

Follow-up paper Vicens 2008 for long term follow-up.

Funding source: Spanish Society of Family and Community Medicine (Grant: 2000/08)

Declaration of interest: None.

\section{Risk of bias}

\begin{tabular}{|c|c|c|}
\hline Bias & Authors' judgement & Support for judgement \\
\hline $\begin{array}{l}\text { Random sequence generation (selection } \\
\text { bias) }\end{array}$ & Low risk & $\begin{array}{l}\text { "Patients were block randomised into two } \\
\text { groups, with one block per physician" }\end{array}$ \\
\hline Allocation concealment (selection bias) & Low risk & Sealed envelopes were used at randomisation. \\
\hline $\begin{array}{l}\text { Blinding (performance bias and detection } \\
\text { bias) } \\
\text { All outcomes }\end{array}$ & Unclear risk & No objective measure used. \\
\hline
\end{tabular}

Blinding of participants and personnel High risk (performance bias)

All outcomes
"Throughout the study the patient's own statement on their use of benzodiazepines was accepted"

In addition the study was not blinded.

Blinding of outcome assessment (detection Unclear risk

No objective measure used.

bias)

All outcomes

Blinding of outcome assessor (detection High risk bias) subjective outcomes

“Throughout the study the patient's own statement on their use of benzodiazepines was accepted"

In addition the study was not blinded 
Vicens 2006 (Continued)

\begin{tabular}{|c|c|c|}
\hline $\begin{array}{l}\text { Incomplete outcome data (attrition bias) } \\
\text { All outcomes }\end{array}$ & Low risk & ITT analysis performed. \\
\hline Selective reporting (reporting bias) & Unclear risk & $\begin{array}{l}\text { The study protocol is not available but the pub- } \\
\text { lished report includes the expected outcome } \\
\text { ("The main efficacy variable was benzodiazepine } \\
\text { use at } 12 \text { months") which was pre-specified in } \\
\text { the methods section. A follow-up at } 36 \text { months } \\
\text { was also carried out which reported the same } \\
\text { outcome measures (Vicens 2008). }\end{array}$ \\
\hline \multicolumn{3}{|l|}{ Vorma 2002} \\
\hline Methods & \multicolumn{2}{|c|}{ RCT. Method of randomisation not reported. } \\
\hline Participants & \multicolumn{2}{|c|}{$\begin{array}{l}62 \text { patients referred by GPs and psychiatrists and supplemented by volunteers answering } \\
\text { advertisements in local newspapers in Finland. Participants had to meet DSM-III R } \\
\text { criteria for BZD dependence } \\
32 \text { participants in intervention group and } 30 \text { participants in control group }\end{array}$} \\
\hline Interventions & \multicolumn{2}{|c|}{$\begin{array}{l}\text { Intervention group: CBT plus taper. This included BZD taper, } 2 \text { weeks stabilisation } \\
1 / 10 \text { per week, taper plan, BZD diaries, education on BZD taper, alternative ways of } \\
\text { coping, progressive relaxation exercises, high risk situations, problem solving, problem } \\
\text { solving for couples, handling sleep, coping with anxiety, coping with depression and } \\
\text { homework assignments } \\
\text { Control group: Standard withdrawal treatment. This included, gradual BZD taper han- } \\
\text { dled by physician, nurse or therapist, diaries of BZD use, supportive therapy as needed, } \\
\text { occasionally brief psychotherapy using strengths perspective }\end{array}$} \\
\hline Outcomes & \multicolumn{2}{|c|}{$\begin{array}{l}\text { Objective urinalysis measures were used to determine BZD discontinuation and dose } \\
\text { reduction at baseline, } 6 \text { month and } 12 \text { month follow-up }\end{array}$} \\
\hline Notes & \multicolumn{2}{|c|}{$\begin{array}{l}\text { Data supplied by author. } \\
\text { There is a paper by Vorma } 2003 \text { also linked to this study. } \\
\text { Funding source: Grants from the Finnish Foundation for Alcohol Studies and the Yrjö } \\
\text { Jahnsson Foundation. Orion Pharma supported the study by supplying the fluoxetine } \\
\text { medication } \\
\text { Declarations of interest: None. }\end{array}$} \\
\hline
\end{tabular}

Risk of bias

\begin{tabular}{l|l|l}
\hline Bias & Authors' judgement & Support for judgement \\
\hline $\begin{array}{l}\text { Random sequence generation (selection } \\
\text { bias) }\end{array}$ & Unclear risk & Method of randomisation not reported. \\
\hline Allocation concealment (selection bias) & Low risk & "The subjects were randomized into two \\
treatments by the sealed envelope method"
\end{tabular}

Psychosocial interventions for benzodiazepine harmful use, abuse or dependence (Review)

Copyright () 2015 The Cochrane Collaboration. Published by John Wiley \& Sons, Ltd. 
Vorma 2002 (Continued)

\begin{tabular}{l|l|l}
$\begin{array}{l}\text { Blinding (performance bias and detection } \\
\text { bias) }\end{array}$ & Low risk & $\begin{array}{l}\text { During treatment, urine BZDs were anal- } \\
\text { ysed monthly and serum BZDs every three }\end{array}$ \\
All outcomes & months to confirm subject compliance
\end{tabular}

months to confirm subject compliance

Blinding of participants and personnel Unclear risk

No subjective outcomes were used.

(performance bias)

All outcomes

Blinding of outcome assessment (detection Low risk bias)

All outcomes

During treatment, urine BZDs were analysed monthly and serum BZDs every three months to confirm subject compliance

Blinding of outcome assessor (detection Unclear risk

No subjective outcomes were used.

bias) subjective outcomes

Incomplete outcome data (attrition bias) Low risk

ITT analysis performed.

All outcomes

Selective reporting (reporting bias) Low risk

The study protocol is not available but the published report includes the expected outcome which was pre-specified in the methods section

Zahradnik 2009

\begin{tabular}{ll} 
Methods & RCT. \\
\hline Participants & $\begin{array}{l}\text { Adult patients between } 18 \text { to } 69 \text { years old admitted to surgical or gynaecological wards } \\
\text { in Germany who had consumed prescription drugs (PD) with addiction potential for } \\
\text { more than } 60 \text { days in last } 3 / 12 \text { or fulfilled DSM IV criteria for PD dependence or abuse. } \\
\text { Drugs considered to have addiction potential; opiates, sedative-hypnotics or caffeine. } 39 \\
\text { participants; } 20 \text { participants in intervention group and } 19 \text { participants in control group }\end{array}$
\end{tabular}

Interventions

Intervention group: MI. One counselling session using MI in hospital lasting 30 to 45 minutes. Four weeks later one counselling session of MI by telephone including assessment of core constructs of cycle of change (readiness to change) and designed individualised written intervention on basis of this assessment which was fed back by letter sent at 8 weeks after first intervention targeted at self-efficacy and maintaining changes. Advised to seek help of GP or medical specialist in reducing medication Control group: Information booklet about problematic prescription drug use

Outcomes

Self-reported BZD use as measured in defined daily doses at baseline and at 3-month follow-up

Notes

Additional data supplied by author.

Follow-up paper with 12-month follow-up Otto 2009.

Funding source: The German research network EARLINT (EARly substance use IN-

Tervention) of the German Federal Ministry of Health (Grant: 15 02/68661) 
Zahradnik 2009 (Continued)

Declaration of interest: None.

Risk of bias

\begin{tabular}{|c|c|c|}
\hline Bias & Authors' judgement & Support for judgement \\
\hline $\begin{array}{l}\text { Random sequence generation (selection } \\
\text { bias) }\end{array}$ & Unclear risk & Method of randomisation not reported. \\
\hline Allocation concealment (selection bias) & High risk & No concealment as randomised wards known to investigators. \\
\hline $\begin{array}{l}\text { Blinding (performance bias and detection } \\
\text { bias) } \\
\text { All outcomes }\end{array}$ & Unclear risk & No objective measures used. \\
\hline $\begin{array}{l}\text { Blinding of participants and personnel } \\
\text { (performance bias) } \\
\text { All outcomes }\end{array}$ & High risk & $\begin{array}{l}\text { No blinding or incomplete blinding, and the outcome is likely } \\
\text { to be influenced by lack of blinding }\end{array}$ \\
\hline $\begin{array}{l}\text { Blinding of outcome assessment (detection } \\
\text { bias) } \\
\text { All outcomes }\end{array}$ & Unclear risk & No objective measures used. \\
\hline $\begin{array}{l}\text { Blinding of outcome assessor (detection } \\
\text { bias) subjective outcomes }\end{array}$ & Low risk & $\begin{array}{l}\text { "A blinded personal interview was conducted by staff who had } \\
\text { no contact with the patient prior to the outcome assessment that } \\
\text { was conducted mainly by telephone" }\end{array}$ \\
\hline $\begin{array}{l}\text { Incomplete outcome data (attrition bias) } \\
\text { All outcomes }\end{array}$ & Low risk & ITT analysis performed. \\
\hline Selective reporting (reporting bias) & Low risk & $\begin{array}{l}\text { The study protocol is not available but the published reports } \\
\text { include all expected outcomes, including those that were pre- } \\
\text { specified in the methods section }\end{array}$ \\
\hline
\end{tabular}

Characteristics of excluded studies [ordered by study ID]

\begin{tabular}{l|l}
\hline Study & Reason for exclusion \\
\hline
\end{tabular}

Ball 2007

Ball 2007 was a multi-site randomised trial of motivational enhancement therapy in community drug clinics in Connecticut, Philadephia, California, USA. There were 461 participants. It was found that motivational enhancement therapy resulted in more sustained substance use reduction than counselling as usual among alcohol users but no difference was found for primary drug users

Excluded as type of interventions were not in the inclusion criteria. There were no participants who received the intervention and were consuming BZDs

Psychosocial interventions for benzodiazepine harmful use, abuse or dependence (Review) 
Bruce $1995 \quad$ Bruce 1995 examined predictors of Alprazolam discontinuation with and without CBT in 20 patients with panic disorder in Illinois, USA. Across groups, reduction in the fear of anxiety symptoms was the best predictor of patients' ability to achieve and maintain drug abstinence

Excluded as no additional outcome data reported.

Bélanger 2005 Bélanger 2005 included 52 older adults with chronic insomnia in Quebec, Canada. Some received CBT and some did not. Compliance with a taper programme and measurement of self efficacy were outcomes evaluated. Those patients who complied with the intervention and became medication free reported higher self efficacy ratings

Excluded as type of outcome data not in the inclusion criteria. Did not measure BZDs over time

Carroll $2009 \quad$ Carroll 2009 conducted a multi-site RCT comparing the effectiveness of three individual sessions of motivational enhancement therapy with three individual sessions of counselling as usual, in 406 Hispanic individuals seeking treatment for any type of current substance use in Florida, New York, Oregon, Colorado and New Mexico. Although both types of intervention resulted in reductions in substance use, there were no significant findings

Excluded as type of interventions were not in the inclusion criteria. There were no participants who received the intervention and were consuming BZDs

Chang 2010

Chang 2010 was a study carried out among 84 homeless veterans with substance use problems in Massachusetts, USA. The participants were randomised to acupuncture, relaxation and usual care. There was no statistical difference between the two interventions but each intervention was significantly different from usual care; for acupuncture there were significantly greater reductions in craving and anxiety levels and greater improvements in the spirituality dimension of quality of life while the relaxation response group had significantly greater reductions in anxiety level and greater improvements in mental health and spirituality dimensions of quality of life

Excluded as the type of participants were not in the inclusion criteria. Patients were already detoxified from all substances before commencing psychosocial intervention. "Homeless veterans with a substance use disorder can be admitted to the domiciliary only after undergoing a detoxification program and remaining substance free for at least 14 days prior to admission. The residents are required to remain sober during their stay in the programme"

Chutuape 1999

Chutuape 1999 included a urinalysis-based contingency management. A methadone take home dose or USD 25 voucher was offered to seven methadone maintained patients, compared to care as usual to seven others. The study was carried out in Baltimore, Maryland, USA. The contingency managed patients submitted significantly more drug free urines than the control patients over a 28 week period

Excluded as the type of participants were not in the inclusion criteria. Patients were already detoxified from all substances before commencing psychosocial intervention. "Following completion of the detox, patients attended the outpatient clinic seven days per week; attended twice-weekly counselling session"

Cormack 1994

Cormack 1994 was a quasi-randomised study, comparing two interventions (letter from a GP and letter from a GP plus four information sheets at monthly intervals) with usual care. The aim was to reduce use of BZDs in 209 chronic users in GP settings in Exeter, England. After six months, both intervention groups had reduced/stopped BZD use to the same extent and significantly greater than the control group Excluded as type of design not in the inclusion criteria. Participants were quasi-randomised. "Within each doctors list, identified users were allocated to the three groups, roughly matched for age and sex to ensure a representative spread between groups. Beyond this, allocation to groups was random"

Psychosocial interventions for benzodiazepine harmful use, abuse or dependence (Review)

Copyright (๑) 2015 The Cochrane Collaboration. Published by John Wiley \& Sons, Ltd. 

psychotherapy were compared with those who received no psychotherapy. The group who received psychotherapy significantly reduced hypnotic consumption at 12 week follow-up

Excluded as participant data not available specifically for BZDs

Godfrey 2008

Godfrey 2008 examined an economic evaluation of two interventions (letter from a GP and consultation with a GP) in long term BZD users in Newcastle, England. Each intervention had been shown to be equally effective. The cost of the consultation was calculated at GBP 40 per patient and the saving per letter patient was GBP 383

Excluded as type of design not in the inclusion criteria. Cost analysis of treatment

Heather 2011

Heather 2011 carried out a logistic regression on data from a previous study looking at predictors of response to brief intervention in general practice against long-term BZD use in 299 individuals in Newcastle, England. Prescription of BZD by own GP as opposed to another doctor and being in the contemplation phase or active phase as opposed to the pre-contemplation phase both predicted a better reduced intake of BZDs. Level of BZD dependence, baseline BZD dosage, type of BZD and gender did not predict a better response Excluded as type of design not in the inclusion criteria. Predictors of relapse

Iguchi 1988 Iguchi 1988 examined reinforcement contingency (weekly take home) plus aversive consequences for unauthorised drug use (reduction of methadone dose) was compared with reinforcement contingency alone, in 16 polydrug using methadone maintained patients in Baltimore, Maryland, USA. No difference was found, supporting previous work

Excluded as participant data not available specifically for BZDs

Jones 1990

Jones 1990 was a RCT which compared 112 elderly patients in two general practice settings in South Wales, UK who had an intervention (GP consultation with nurse reinforcement, counselling and relaxation therapy) for chronic psychotropic use (mostly BZD) with 115 patients who had no documented intervention. There was a significantly greater reduction in those who had reduced or stopped psychotropic use at nine month follow-up Excluded as participant data not available specifically for BZDs

Lichstein 2013 Lichstein 2013 examined CBT plus drug withdrawal, placebo biofeedback plus withdrawal or drug withdrawal only, in 70 patients in Tennessee, USA. Only the CBT group showed significant change in some outcomes. There were no significant differences in drug reduction and BZDs could not be disaggregated 

part of a study to measure reduction in long-term BZD use in general practice in the Netherlands. This study showed that BZD craving severity decreased over time, patients still using BZDs demonstrated significantly more craving than those who had quit and patients who had received additional tapering reported significantly more craving than those who had only received a letter as an incentive to quit Excluded as type of outcomes not in the inclusion criteria. Control data not reported and not available part of a study to measure reduction in long-term BZD use in general practice in the Netherlands. This study showed that BZD craving severity decreased over time, patients still using BZDs demonstrated significantly more craving than those who had quit and patients who had received additional tapering reported significantly more craving than those who had only received a letter as an incentive to quit

Excluded as type of outcome not in the inclusion criteria. Cross sectional craving data only hypnotic users. The study was carried out in 209 patients in routine general practice settings in Sheffield, England. The package of six CBT sessions was compared with usual treatment. CBT-treated patients reported reduced consumption of hypnotics. A range of other outcomes were improved also. The total cost of the intervention was 154.40 pounds. The mean incremental cost per quality-adjusted life year at six months was 3418 pounds

Excluded as participant data not available specifically for BZDs ments as a means of reducing BZD dosage in 18 individuals in London, UK taking BZDs for longer than four months. A greater reduction occurred in the group training arm at 6 -week follow-up but the reduction was greater in the GP arm after 15 months

Excluded as type of design not in the inclusion criteria. Participants were quasi-randomised. "Subjects were allocated to either a group training or individual appointment condition. Allocation was randomised as far as possible, although time constraints and a slow rate of volunteering meant that the group condition was filled first"

Otto 1992

Otto 1992 presented a cognitive behavioural conceptualisation of BZD discontinuation difficulties, emphasizing 'fear of fear' cycles, which was conducted in Massachusetts, USA. The discontinuation process is seen as exposing panic disorder patients to somatic sensations associated with panic. The paper did not contain any quantitative data

Excluded as the study design was not part of the review. Conceptual paper, no relevant data

Oude Voshaar 2003 Oude Voshaar 2003 comprised a cross-validation and assessment of predictive validity of the BZD dependence self-report questionnaire (Bendep-SRQ) in a BZD trial involving 180 chronic BZD users in the Netherlands. All scales showed excellent reliability while construct and discriminant validity were adequate. All four scales contributed significantly to the prediction of whether complete abstinence would be achieved. The authors recommended use of the Bendep-SRQ in discontinuation therapy Excluded as type of design not in the inclusion criteria. Predictors of relapse and validation of a measure

Oude Voshaar 2006a Oude Voshaar 2006a compared the relative costs of tapering off long-term BZD use combined with group CBT $(\mathrm{TO}+\mathrm{CBT})$, tapering off alone (TOA) and usual care (UC). The setting was primary care across the Netherlands and there were 180 chronic BZD users participating. Cost and effectiveness data were assessed. 
Intervention treatment costs averaged 172.99 Euro per patient undergoing TO + CBT and 69.5 Euro per patient undergoing TOA. The incremental cost-effectiveness ratios (ICERs) showed that, for each $1 \%$ successful BZD discontinuation, TO+CBT had ICERs in the range of 10.30 to 62.53 Euro versus UC, depending on the study perspective while the range for TOA versus UC was 0.57 to 48.92 Euro Excluded as type of design not in the inclusion criteria. Predictors of abstinence

Oude Voshaar 2006b Oude Voshaar 2006b identified predictors of successful discontinuation in a BZD discontinuation trial in 180 patients across primary care settings in the Netherlands. Independent predictors of success were; offering a taper-off programme with or without group therapy, a lower BZD dose at the start of tapering, less severe BZD dependence and no use of alcohol

Excluded as type of design not in the inclusion criteria. Economic evaluation of treatment

Pollack 2002 Pollack 2002 examined a novel CBT, targeting the reduction of sensitivity to interoceptive cues associated with drug craving and trained alternatives to the cues (CBT) which was compared to increased counselling in 23 opiate-dependent patients in Boston, Massachusetts, USA over a 6-month follow-up period. There was a trend towards reduced drug usage in women and the opposite trend in men

Excluded as participant data not available specifically for BZDs

Salonoja 2010

Salonoja 2010 conducted a study in which the setting for and participants in this study were 591 community dwelling people aged 65 or older in Finland who were chronic BZD or related drug (RD) users. The intervention comprised instruction to withdraw, reduce or change psychotropic drugs, supplemented by a one hour lecture. This was compared to usual care with no suggested changes in drug therapy. There was a statistically significant difference in reduction of drug consumption, favouring the intervention group. Results for BZDs and RDs could not be differentiated in the paper Excluded as participant data not available specifically for BZDs

Scherzer 1996 Scherzer 1996 formed part of a thesis in The Union Institute, Ohio, USA. Traditional treatment (TT) consisted of eight sessions of CBT, imaginal exposure and relaxation. Thermal and galvanic skin response data were recorded in the TT group but no feedback was given. Experimental treatment (ET) group had all the above plus biofeedback. Results indicated a significant decrease in time needed to discontinue dependence on medication for the ET group. The ET group also showed lower levels of generalised anxiety, depression, anticipatory anxiety and intensity of panic sensations. These effects were maintained at 6 month follow-up Excluded as participant data not available specifically for BZDs

Soeffing 2008

Soeffing 2008 examined sleep outcomes in 47 hypnotic-dependent older people in Tennessee, USA. Interventions included CBT, comprising relaxation training, stimulus control and sleep hygiene instructions. Patients were instructed to stay on fixed amounts of medication during the study so drug changes were excluded as an outcome measure

Excluded as type of outcome not in the inclusion criteria. "Participants were instructed not to alter their pattern of hypnotic consumption during treatment"

Stitzer 1992

Stitzer 1992 examined contingent methadone take-home privileges for effectiveness in reducing on-going supplemental drug use in methadone maintenance patients in Maryland, USA. New intake patients $(\mathrm{N}=$ 53) were randomly assigned to receiving take-home privileges based on urine results or to a non-contingent procedure in which take-homes were delivered independently of urine results. The contingent procedure produced a significantly higher rate of drug free urines over a 4 week follow-up period Excluded as participant data not available specifically for BZDs

Psychosocial interventions for benzodiazepine harmful use, abuse or dependence (Review) 

Sleep restriction therapy and hypnotic withdrawal was compared with sleep hygiene education. Hypnotic withdrawal was an intervention rather than an outcome and not all participants were using hypnotics- $82 \%$ were. Hypnotics were not characterized and objective measures were not used Excluded as participant data not available specifically for BZDs

Vorma 2005

Vorma 2005 examined 76 patients manifesting complicated BZD dependence who were part of a RCT to assess predictors of BZD discontinuation carried out in Finland. People with lower BZD doses and no previous attempt at withdrawal were more successful at BZD discontinuation. Cluster B personality/ borderline personality disorder was associated with an inability to stop BZD use

Excluded as type of design not in the inclusion criteria. Predictors of abstinence 
DATA AND ANALYSES

\section{Comparison 1. CBT (plus taper) versus taper}

\begin{tabular}{|c|c|c|c|c|}
\hline Outcome or subgroup title & $\begin{array}{l}\text { No. of } \\
\text { studies }\end{array}$ & $\begin{array}{c}\text { No. of } \\
\text { participants }\end{array}$ & Statistical me & Effect size \\
\hline $\begin{array}{l}1 \text { Successful discontinuation of } \\
\text { BZDs }\end{array}$ & 11 & & Risk Ratio (M-H, Random, 95\% CI) & Subtotals only \\
\hline 1.1 Post treatment & 9 & 423 & Risk Ratio (M-H, Random, 95\% CI) & $1.40[1.05,1.86]$ \\
\hline 1.23 month follow-up & 9 & 460 & Risk Ratio (M-H, Random, 95\% CI) & $1.51[1.15,1.98]$ \\
\hline 1.36 month follow-up & 3 & 155 & Risk Ratio (M-H, Random, 95\% CI) & $1.94[0.88,4.30]$ \\
\hline $1.411 / 12$ month follow-up & 5 & 284 & Risk Ratio (M-H, Random, 95\% CI) & $1.42[0.89,2.28]$ \\
\hline 1.515 month follow-up & 1 & 146 & Risk Ratio (M-H, Random, 95\% CI) & $0.8[0.49,1.31]$ \\
\hline 1.6 Follow-up $\geq 24$ months & 2 & 73 & Risk Ratio (M-H, Random, 95\% CI) & $1.77[0.98,3.17]$ \\
\hline $\begin{array}{l}2 \text { Sensitivity analysis (Allocation } \\
\text { concealment): successful } \\
\text { discontinuation of BZDs }\end{array}$ & 8 & & Risk Ratio (M-H, Random, 95\% CI) & Subtotals only \\
\hline 2.1 Post treatment & 8 & 370 & Risk Ratio (M-H, Random, 95\% CI) & $1.50[1.12,2.02]$ \\
\hline $\begin{array}{l}3 \text { Sensitivity analysis (Blinding } \\
\text { of assessor): successful } \\
\text { discontinuation of BZDs }\end{array}$ & 5 & & Risk Ratio (M-H, Random, 95\% CI) & Subtotals only \\
\hline 3.1 Post treatment & 4 & 159 & Risk Ratio (M-H, Random, 95\% CI) & $1.08[0.73,1.59]$ \\
\hline 3.23 month follow-up & 4 & 103 & Risk Ratio (M-H, Random, 95\% CI) & $1.60[1.08,2.36]$ \\
\hline 3.36 month follow-up & 2 & 94 & Risk Ratio (M-H, Random, 95\% CI) & $1.98[0.37,10.47]$ \\
\hline 3.412 month follow-up & 1 & 62 & Risk Ratio (M-H, Random, 95\% CI) & $0.70[0.28,1.79]$ \\
\hline 3.5 Follow-up $\geq 24$ months & 1 & 21 & Risk Ratio (M-H, Random, 95\% CI) & $2.73[1.02,7.32]$ \\
\hline 4 Reduce BZDs > 50\% & 4 & & Odds Ratio (M-H, Random, 95\% CI) & Subtotals only \\
\hline 4.1 Post treatment & 3 & 178 & Odds Ratio (M-H, Random, 95\% CI) & $0.93[0.11,8.18]$ \\
\hline 4.23 month follow-up & 2 & 69 & Odds Ratio (M-H, Random, 95\% CI) & $1.99[0.47,8.47]$ \\
\hline 4.36 month follow-up & 1 & 62 & Odds Ratio (M-H, Random, 95\% CI) & $0.76[0.28,2.07]$ \\
\hline 4.412 month follow-up & 2 & 125 & Odds Ratio (M-H, Random, 95\% CI) & $1.07[0.14,8.21]$ \\
\hline 5 Drop-outs or lost to follow & 10 & & Risk Ratio (M-H, Random, 95\% CI) & Subtotals only \\
\hline $\begin{array}{l}\text { 5.1 From } 0 \text { to post treatment } \\
\text { follow-up }\end{array}$ & 9 & 478 & Risk Ratio (M-H, Random, 95\% CI) & $1.05[0.66,1.66]$ \\
\hline $\begin{array}{l}5.2 \text { From } 0 \text { to } 3 \text { month } \\
\text { follow-up }\end{array}$ & 1 & 65 & Risk Ratio (M-H, Random, 95\% CI) & $1.71[0.16,17.98]$ \\
\hline $\begin{array}{l}5.3 \text { From } 0 \text { to } 6 \text { month } \\
\text { follow-up }\end{array}$ & 1 & 62 & Risk Ratio (M-H, Random, 95\% CI) & $0.70[0.17,2.88]$ \\
\hline $\begin{array}{l}\text { 5.4 From } 0 \text { to } 12 \text { month } \\
\text { follow-up }\end{array}$ & 1 & 65 & Risk Ratio (M-H, Random, 95\% CI) & $2.57[0.28,23.44]$ \\
\hline
\end{tabular}

Psychosocial interventions for benzodiazepine harmful use, abuse or dependence (Review) 


\begin{tabular}{lccll} 
Outcome or subgroup title & $\begin{array}{c}\text { No. of } \\
\text { studies }\end{array}$ & $\begin{array}{c}\text { No. of } \\
\text { participants }\end{array}$ & \multicolumn{1}{c}{ Statistical method } & Effect size \\
\hline $\begin{array}{l}\text { 1 Successful discontinuation of } \\
\text { BZDs }\end{array}$ & 4 & & Risk Ratio (M-H, Random, 95\% CI) & Subtotals only \\
1.1 Post treatment & 2 & 34 & Risk Ratio (M-H, Random, 95\% CI) & $4.43[0.16,125.35]$ \\
1.23 month follow-up & 4 & 80 & Risk Ratio (M-H, Random, 95\% CI) & $3.46[0.53,22.45]$ \\
$\begin{array}{l}\text { Reduce BZD > 50\% } \\
2.13 \text { month follow-up }\end{array}$ & 1 & & Risk Ratio (M-H, Fixed, 95\% CI) & Subtotals only \\
2.212 month follow-up & 1 & 39 & Risk Ratio (M-H, Fixed, 95\% CI) & $1.52[0.60,3.83]$ \\
\end{tabular}

\section{Comparison 3. Standardised interview (taper) versus TAU}

\begin{tabular}{lcccc} 
Outcome or subgroup title & $\begin{array}{c}\text { No. of } \\
\text { studies }\end{array}$ & $\begin{array}{c}\text { No. of } \\
\text { participants }\end{array}$ & Statistical method & Effect size \\
\hline $\begin{array}{l}\text { 1 Successful discontinuation of } \\
\text { BZDs }\end{array}$ & 1 & & Risk Ratio (M-H, Fixed, 95\% CI) & Subtotals only \\
1.16 month follow-up & 1 & 139 & Risk Ratio (M-H, Fixed, 95\% CI) & $13.11[3.25,52.83]$ \\
1.212 month follow-up & 1 & 139 & Risk Ratio (M-H, Fixed, 95\% CI) & $4.97[2.23,11.11]$ \\
1.3 3 year follow-up & 1 & 139 & Risk Ratio (M-H, Fixed, 95\% CI) & $1.61[0.92,2.84]$ \\
$\begin{array}{l}\text { Reduce BZD > 50\% } \\
2.16 \text { month follow-up }\end{array}$ & 1 & & Risk Ratio (M-H, Fixed, 95\% CI) & Subtotals only \\
2.212 month follow-up & 1 & 139 & Risk Ratio (M-H, Fixed, 95\% CI) & $3.32[1.43,7.67]$ \\
\end{tabular}

Comparison 4. CBT (no taper) versus TAU

\begin{tabular}{ccccc}
\hline & & & & \\
Outcome or subgroup title & $\begin{array}{c}\text { No. of } \\
\text { studies }\end{array}$ & $\begin{array}{c}\text { No. of } \\
\text { participants }\end{array}$ & Statistical method & Effect size \\
\hline 1 BZD positive urine rate & 1 & & Mean Difference (IV, Fixed, 95\% CI) & Subtotals only \\
1.1 Post treatment & 1 & 73 & Mean Difference (IV, Fixed, 95\% CI) & $-0.01[-0.19,0.17]$ \\
1.23 month follow-up & 1 & 73 & Mean Difference (IV, Fixed, 95\% CI) & $-0.08[-0.25,0.09]$ \\
1.36 month follow-up & 1 & 73 & Mean Difference (IV, Fixed, 95\% CI) & $-0.09[-0.25,0.07]$ \\
\hline
\end{tabular}




\begin{tabular}{lcclc} 
Outcome or subgroup title & $\begin{array}{c}\text { No. of } \\
\text { studies }\end{array}$ & $\begin{array}{c}\text { No. of } \\
\text { participants }\end{array}$ & Statistical method & Effect size \\
\hline $\begin{array}{l}\text { 1 Change in OTI score for BZD } \\
\quad 1\end{array}$ & & Mean Difference (IV, Fixed, 95\% CI) & Subtotals only \\
$\begin{array}{l}\text { use } \\
\text { 1.1 Post treatment }\end{array}$ & 1 & 29 & Mean Difference (IV, Fixed, 95\% CI) & $0.73[-1.94,3.40]$ \\
1.26 months follow-up & 1 & 24 & Mean Difference (IV, Fixed, 95\% CI) & $-0.27[-4.06,3.52]$ \\
\hline
\end{tabular}

Comparison 6. Complaints management (additional relaxation) versus anxiety management (relaxation)

\begin{tabular}{lcclc} 
Outcome or subgroup title & $\begin{array}{c}\text { No. of } \\
\text { studies }\end{array}$ & $\begin{array}{c}\text { No. of } \\
\text { participants }\end{array}$ & Statistical method & Effect size \\
\hline $\begin{array}{l}\text { 1 Successful discontinuation of } \\
\quad 1\end{array}$ & & Risk Ratio (M-H, Fixed, 95\% CI) & Subtotals only \\
$\quad \begin{array}{l}\text { BZDs } \\
1.1 \text { Post treatment }\end{array}$ & 1 & 19 & Risk Ratio (M-H, Fixed, 95\% CI) & $1.56[0.76,3.17]$ \\
1.26 month follow-up & 1 & 19 & Risk Ratio (M-H, Fixed, 95\% CI) & $0.93[0.43,2.01]$ \\
\hline
\end{tabular}

Comparison 7. Consultation (plus letter) versus TAU

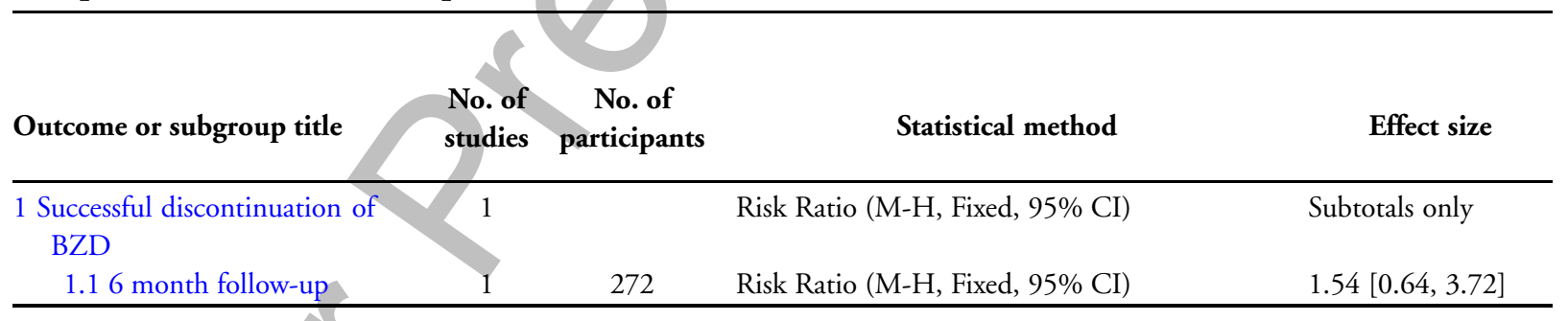

Comparison 8. E-counselling versus onsite counselling

\begin{tabular}{lcccc}
\hline & & & & \\
Outcome or subgroup title & $\begin{array}{c}\text { No. of } \\
\text { studies }\end{array}$ & $\begin{array}{c}\text { No. of } \\
\text { participants }\end{array}$ & Statistical method & Effect size \\
\hline $\begin{array}{c}1 \text { Positive BZD urine toxicology } \\
1.16 \text { week follow-up }\end{array}$ & 1 & & Mean Difference (IV, Fixed, 95\% CI) & $\begin{array}{c}\text { Subtotals only } \\
-0.01[-0.04,0.02]\end{array}$ \\
\hline
\end{tabular}




\begin{tabular}{lcclc} 
Outcome or subgroup title & $\begin{array}{c}\text { No. of } \\
\text { studies }\end{array}$ & $\begin{array}{c}\text { No. of } \\
\text { participants }\end{array}$ & Statistical method & Effect size \\
\hline $\begin{array}{l}\text { 1 Successful discontinuation of } \\
\text { BZDs }\end{array}$ & 1 & & Risk Ratio (M-H, Fixed, 95\% CI) & Subtotals only \\
$\begin{array}{l}1.1 \text { Post treatment } \\
1.23 \text { month follow-up }\end{array}$ & 1 & 60 & Risk Ratio (M-H, Fixed, 95\% CI) & $1.90[0.98,3.70]$ \\
\end{tabular}

\section{Comparison 10. Tailored letter versus GP letter}

\begin{tabular}{lcccc} 
Outcome or subgroup title & $\begin{array}{c}\text { No. of } \\
\text { studies }\end{array}$ & $\begin{array}{c}\text { No. of } \\
\text { participants }\end{array}$ & Statistical method & Effect size \\
\hline $\begin{array}{l}\text { 1 Successful discontinuation of } \\
\text { BZDs }\end{array}$ & 1 & & Risk Ratio (M-H, Fixed, 95\% CI) & Subtotals only \\
1.112 month follow-up & 1 & 322 & Risk Ratio (M-H, Fixed, 95\% CI) & $1.70[1.07,2.70]$ \\
\hline
\end{tabular}

\section{Comparison 11. Relaxation (plus taper) versus taper}

\begin{tabular}{lclll} 
Outcome or subgroup title & $\begin{array}{c}\text { No. of } \\
\text { studies }\end{array}$ & $\begin{array}{c}\text { No. of } \\
\text { participants }\end{array}$ & Statistical method & Effect size \\
\hline $\begin{array}{l}\text { 1 Successful discontinuation of } \\
\text { BZDs }\end{array}$ & 1 & & Risk Ratio (M-H, Fixed, 95\% CI) & Subtotals only \\
1.1 Post treatment & 1 & 31 & Risk Ratio (M-H, Fixed, 95\% CI) & $0.78[0.30,2.03]$ \\
1.23 month follow-up & 1 & 31 & Risk Ratio (M-H, Fixed, 95\% CI) & $0.47[0.10,2.20]$ \\
1.36 month follow-up & 1 & 31 & Risk Ratio (M-H, Fixed, 95\% CI) & $0.47[0.10,2.20]$ \\
\hline
\end{tabular}

Comparison 12. Enhanced skills training (relaxation) versus limited skills training (relaxation)

\begin{tabular}{lcccc}
\hline & & & \\
Outcome or subgroup title & $\begin{array}{c}\text { No. of } \\
\text { studies }\end{array}$ & $\begin{array}{c}\text { No. of } \\
\text { participants }\end{array}$ & Statistical method & Effect size \\
\hline $\begin{array}{l}1 \text { Change in prescribed diazepam } \\
\text { dose (mg) }\end{array}$ & 1 & & Mean Difference (IV, Fixed, 95\% CI) & Subtotals only \\
1.16 month follow-up & 1 & 53 & Mean Difference (IV, Fixed, 95\% CI) & $4.4[-0.01,8.81]$ \\
\hline
\end{tabular}




\section{Analysis I.I. Comparison I CBT (plus taper) versus taper, Outcome I Successful discontinuation of BZDs.}

Review: Psychosocial interventions for benzodiazepine harmful use, abuse or dependence

Comparison: I CBT (plus taper) versus taper

Outcome: I Successful discontinuation of BZDs

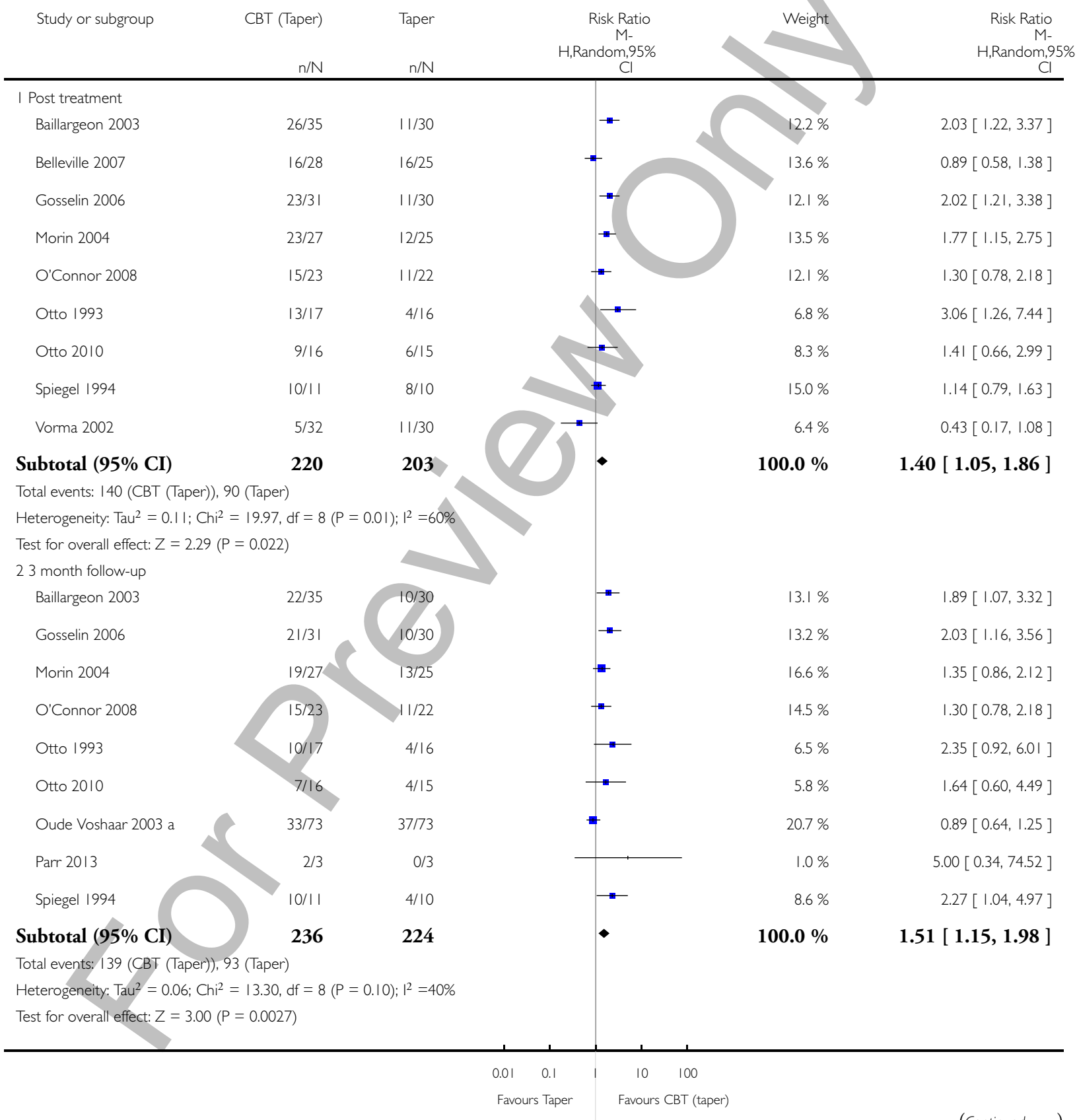

(Continued....)

Psychosocial interventions for benzodiazepine harmful use, abuse or dependence (Review) 


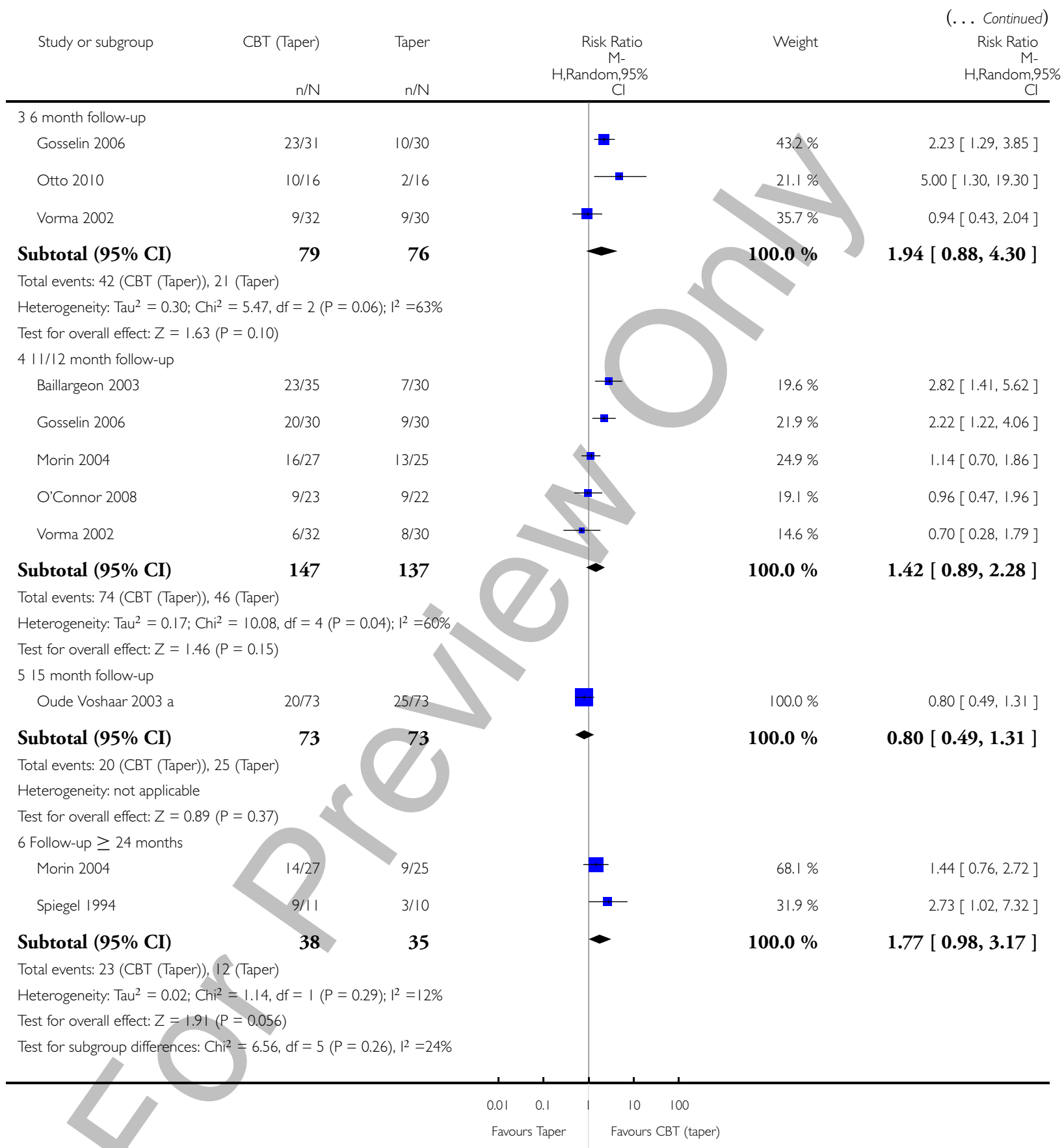




\section{Analysis I.2. Comparison I CBT (plus taper) versus taper, Outcome 2 Sensitivity analysis (Allocation}

concealment): successful discontinuation of BZDs.

Review: Psychosocial interventions for benzodiazepine harmful use, abuse or dependence

Comparison: I CBT (plus taper) versus taper

Outcome: 2 Sensitivity analysis (Allocation concealment): successful discontinuation of BZDs

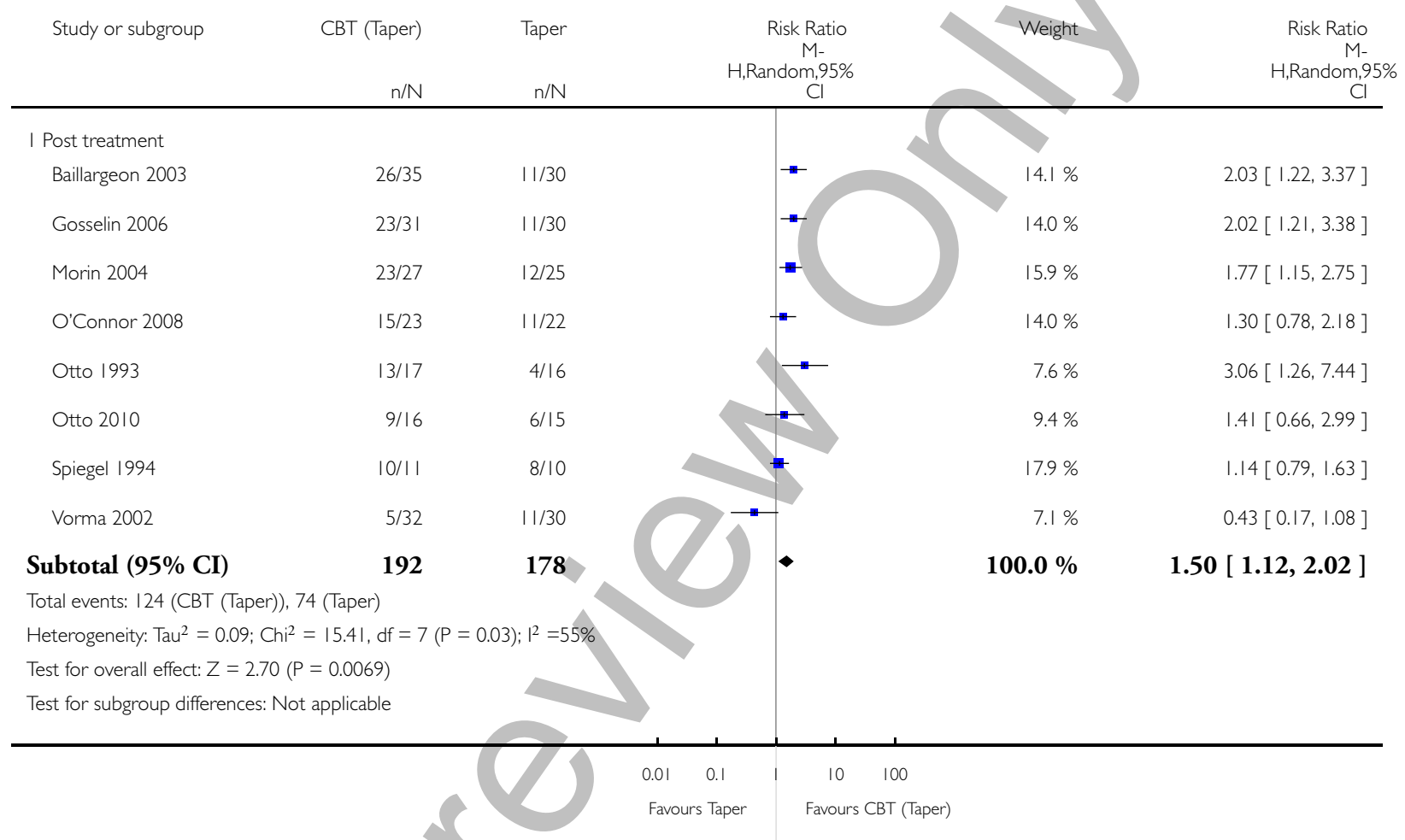


Analysis I.3. Comparison I CBT (plus taper) versus taper, Outcome 3 Sensitivity analysis (Blinding of assessor): successful discontinuation of BZDs.

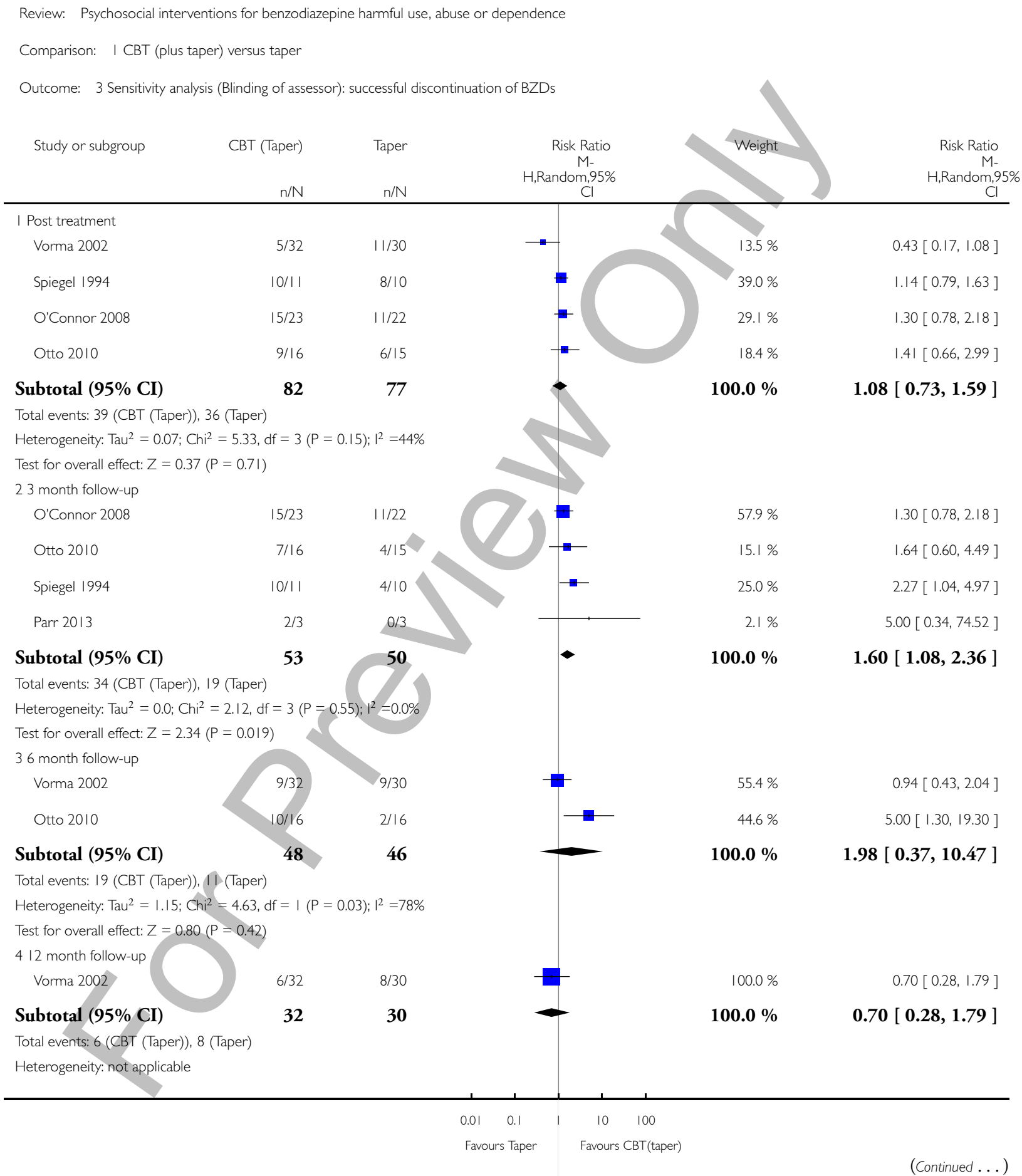

$\begin{array}{lcr} & \mathrm{n} / \mathrm{N} & \mathrm{n} / \mathrm{N} \\ \begin{array}{lcr}\text { I Post treatment } \\ \text { Vorma 2002 }\end{array} & 5 / 32 & 11 / 30 \\ \text { Spiegel 1994 } & 10 / 11 & 8 / 10 \\ \text { O'Connor 2008 } & 15 / 23 & 11 / 22 \\ \text { Otto 2010 } & 9 / 16 & 6 / 15\end{array}$

Subtotal $(95 \% \mathrm{CI})$

$82 \quad 77$

$\mathrm{Cl}$

Weight

Total events: 39 (CBT (Taper)), 36 (Taper)

Heterogeneity: $\operatorname{Tau}^{2}=0.07 ; \mathrm{Chi}^{2}=5.33, \mathrm{df}=3(\mathrm{P}=0.15) ; \mathbf{1}^{2}=44 \%$

Test for overall effect: $Z=0.37(P=0.71)$

23 month follow-up

O'Connor 2008

$15 / 23$

Otto 2010

$7 / 16$

Spiegel 1994

$10 / 11$

Parr 2013

$$
2 / 3
$$

Subtotal (95\% CI)

53

50

Total events: 34 (CBT (Taper)), 19 (Taper)

Heterogeneity: $\mathrm{Tau}^{2}=0.0 ; \mathrm{Chi}^{2}=2.12, \mathrm{df}=3(\mathrm{P}=0.55) ; 1^{2}=0.0 \%$

Test for overall effect: $Z=2.34(P=0.019)$

36 month follow-up

Vorma 2002

Otto 2010

Subtotal (95\% CI)

Total events: 19 (CBT (Taper)), 11 (Taper)

Heterogeneity: $\operatorname{Tau}^{2}=1.15 ; \mathrm{Chi}^{2}=4.63, \mathrm{df}=$ I $(P=0.03) ; \mathrm{I}^{2}=78 \%$

Test for overall effect: $Z=0.80(P=0.42)$

412 month follow-up

Vorma 2002

Subtotal (95\% CI)

Total events: 6 (CBT (Taper)), 8 (Taper)

Heterogeneity: not applicable

6/32 $\quad 8 / 30$

32

10/16 2/16

48

46

\section{0}

16

$\rightarrow$

10

5

7

(n)

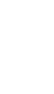

$0.43[0.17,1.08]$

$1.14[0.79,1.63]$

$1.30[0.78,2.18]$

$29.1 \%$

$18.4 \%$

I.41 [0.66, 2.99]

$100.0 \%$

$1.08[0.73,1.59]$

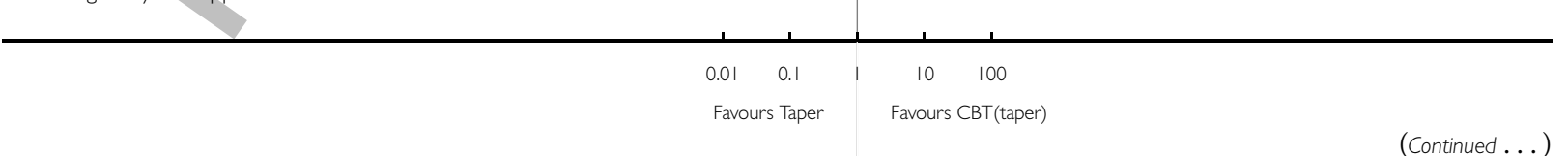

Psychosocial interventions for benzodiazepine harmful use, abuse or dependence (Review) 


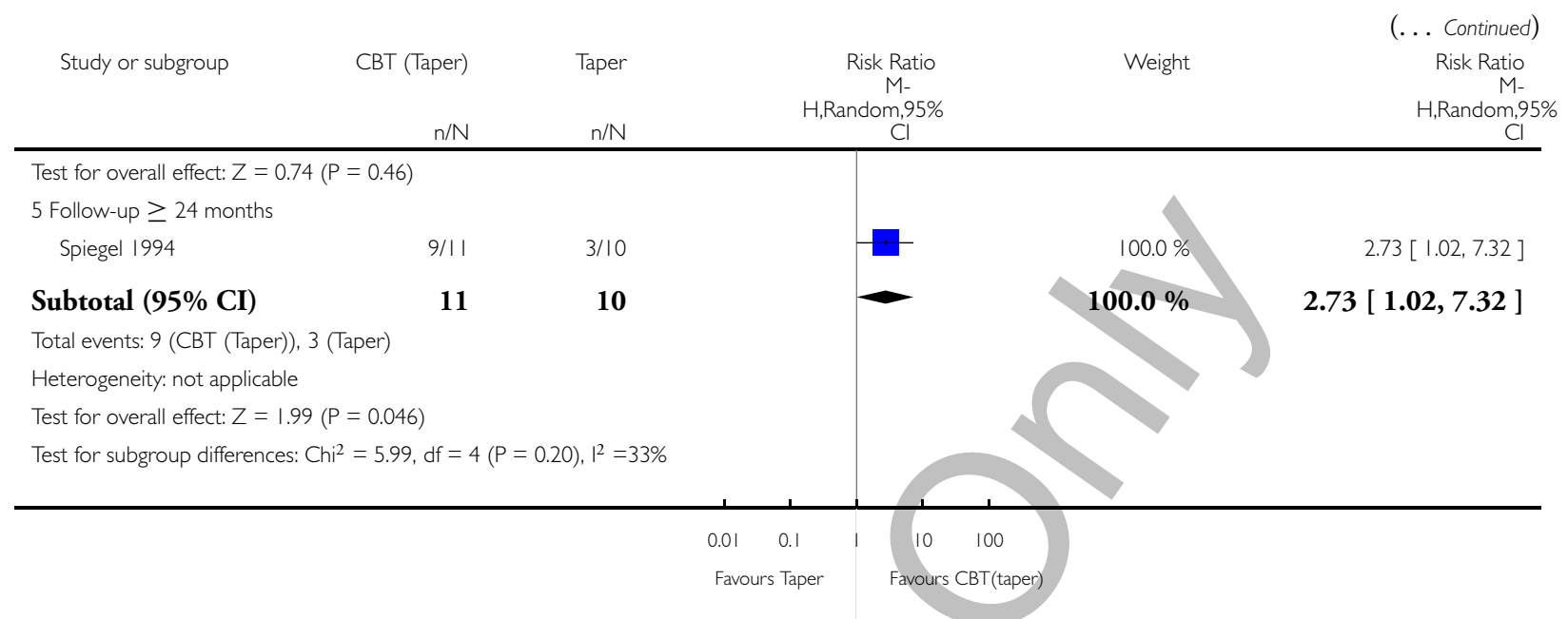

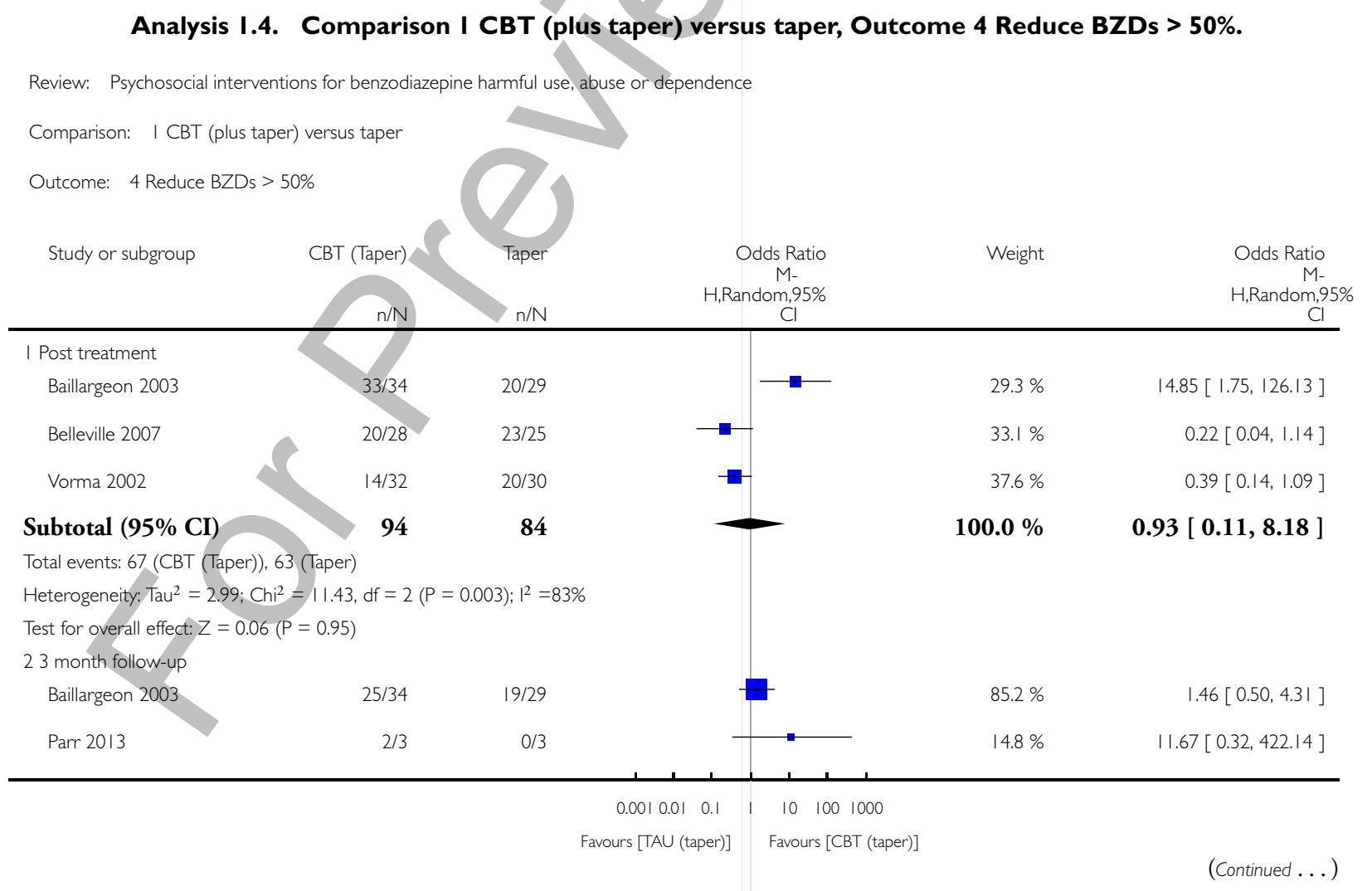

Psychosocial interventions for benzodiazepine harmful use, abuse or dependence (Review)

Copyright @ 2015 The Cochrane Collaboration. Published by John Wiley \& Sons, Ltd. 


\begin{tabular}{|c|c|c|c|c|c|}
\hline Study or subgroup & CBT (Taper) & $\begin{array}{l}\text { Taper } \\
\text { n/N }\end{array}$ & $\begin{array}{r}\text { Odds Ratio } \\
\text { M- } \\
\text { H,Random,95\% } \\
\text { Cl }\end{array}$ & Weight & $\begin{array}{c}\text { (... Continued) } \\
\text { Odds Ratio } \\
\text { M- } \\
\text { H,Random,95\% } \\
\text { Cl }\end{array}$ \\
\hline Subtotal $(95 \% \mathrm{CI})$ & 37 & 32 & & $100.0 \%$ & $1.99[0.47,8.47]$ \\
\hline \multicolumn{6}{|c|}{ Total events: 27 (CBT (Taper)), 19 (Taper) } \\
\hline \multicolumn{6}{|c|}{ Heterogeneity: $\mathrm{Tau}^{2}=0.34 ; \mathrm{Chi}^{2}=1.18, \mathrm{df}=1(P=0.28) ; \mathrm{I}^{2}=16 \%$} \\
\hline \multicolumn{6}{|c|}{ Test for overall effect: $Z=0.93(P=0.35)$} \\
\hline \multicolumn{6}{|l|}{36 month follow-up } \\
\hline Vorma 2002 & $17 / 32$ & $18 / 30$ & & & $0.76[0.28,2.07]$ \\
\hline Subtotal $(95 \% \mathrm{CI})$ & 32 & 30 & & $100.0 \%$ & $0.76[0.28,2.07]$ \\
\hline \multicolumn{6}{|c|}{ Total events: 17 (CBT (Taper)), 18 (Taper) } \\
\hline \multicolumn{6}{|c|}{ Heterogeneity: not applicable } \\
\hline \multicolumn{6}{|c|}{ Test for overall effect: $Z=0.55(P=0.59)$} \\
\hline \multicolumn{6}{|l|}{412 month follow-up } \\
\hline Baillargeon 2003 & $26 / 34$ & $15 / 29$ & & $49.8 \%$ & $3.03[1.03,8.90]$ \\
\hline Vorma 2002 & $15 / 32$ & $21 / 30$ & & $50.2 \%$ & $0.38[0.13,1.08]$ \\
\hline Subtotal (95\% CI) & 66 & 59 & & $100.0 \%$ & $1.07[0.14,8.21]$ \\
\hline \multicolumn{6}{|c|}{ Total events: 4 I (CBT (Taper)), 36 (Taper) } \\
\hline \multicolumn{6}{|c|}{ Heterogeneity: $\operatorname{Tau}^{2}=1.87 ; \mathrm{Chi}^{2}=7.40, \mathrm{df}=1(P=0.01) ;\left.\right|^{2}=86 \%$} \\
\hline \multicolumn{6}{|c|}{ Test for overall effect: $Z=0.06(P=0.95)$} \\
\hline Test for subgroup differenc & $=1.16, \mathrm{df}=3$ & 6), $\left.\right|^{2}=0.0 \%$ & & & \\
\hline
\end{tabular}

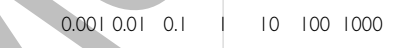

Favours [TAU (taper)] Favours [CBT (taper)] 


\section{Analysis I.5. Comparison I CBT (plus taper) versus taper, Outcome 5 Drop-outs or lost to follow-up.}

Review: Psychosocial interventions for benzodiazepine harmful use, abuse or dependence

Comparison: I CBT (plus taper) versus taper

Outcome: 5 Drop-outs or lost to follow-up

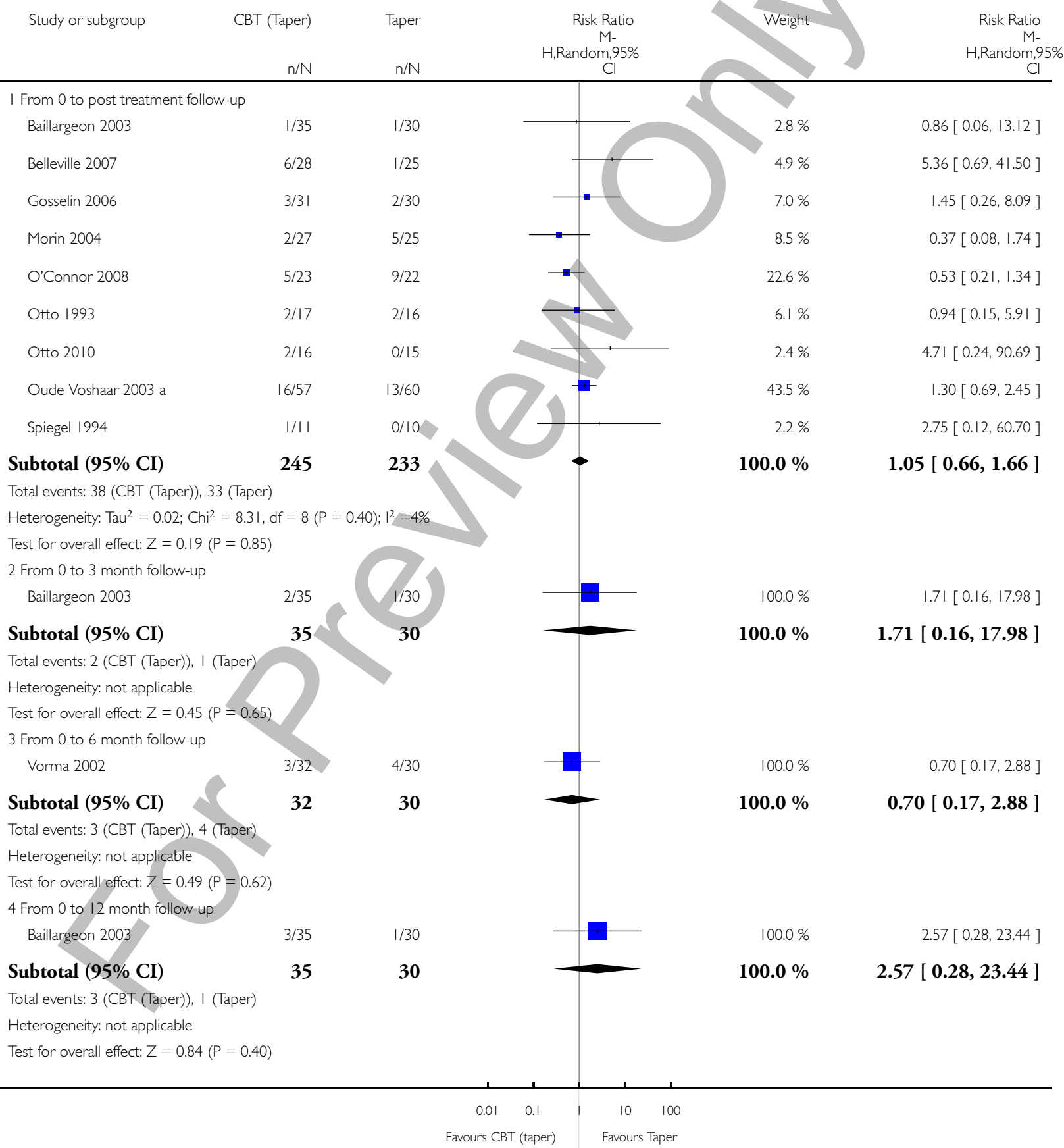

Psychosocial interventions for benzodiazepine harmful use, abuse or dependence (Review)

Copyright @ 2015 The Cochrane Collaboration. Published by John Wiley \& Sons, Ltd. 


\section{Analysis 2.I. Comparison 2 MI versus TAU, Outcome I Successful discontinuation of BZDs.}

Review: Psychosocial interventions for benzodiazepine harmful use, abuse or dependence

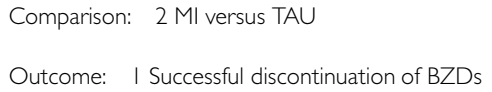

\begin{tabular}{lcr} 
& $\mathrm{n} / \mathrm{N}$ & $\mathrm{n} / \mathrm{N}$ \\
\hline $\begin{array}{c}\text { I Post treatment } \\
\text { Becka 2004 }\end{array}$ & $9 / 16$ & $0 / 15$ \\
Carroll 2006 & $1 / 1$ & |/2
\end{tabular}

\section{Carroll 2006}

$1 / 1$

$1 / 2$

17

17

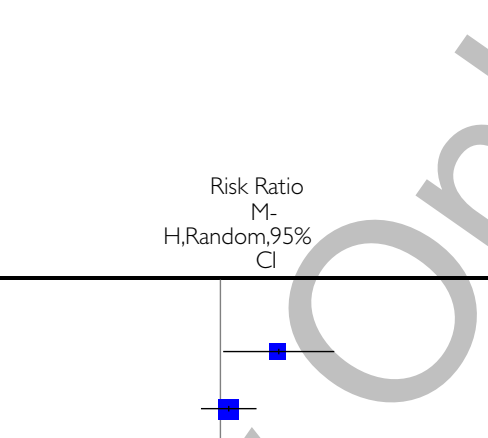

\section{Subtotal (95\% CI)}

Total events: 10 (MI), I (TAU)

Heterogeneity: $\mathrm{Tau}^{2}=4.67 ; \mathrm{Chi}^{2}=4.76, \mathrm{df}=\mathrm{I}(\mathrm{P}=0.03) ; \mathrm{I}^{2}=79 \%$

Test for overall effect: $Z=0.87(P=0.38)$

23 month follow-up

Zahradnik 2009

Becka 2004

Carroll 2006

Bag ien 2013

Subtotal (95\% CI)

Total events: 20 (MI), 4 (TAU)

Heterogeneity: $\mathrm{Tau}^{2}=2.67 ; \mathrm{Chi}^{2}=15.78, \mathrm{df}=3(\mathrm{P}=0.001) ; \mathrm{I}^{2}=81 \%$

Test for overall effect: $Z=1.30(P=0.19)$

Test for subgroup differences: $C h i^{2}=0.02, d f=1(P=0.90), I^{2}=0.0 \%$

Weight

Risk Ratio

$\mathrm{H}$, Random, $95 \%$

$43.7 \%$

$56.3 \%$

$100.0 \%$

$4.43[0.16,125.35]$
$17.88[1.13,282.72]$

$1.50[0.38,6.00]$
$19.1 \%$
$10.48[0.62,177.44]$
$19.6 \%$
$28.7 \%$
$21.65[1.39,337.90]$
$1.50[0.38,6.00]$
$32.6 \%$
$1.25[0.63,2.47]$

$100.0 \%$

3.46 [ 0.53, 22.45 ] 
Analysis 2.2. Comparison 2 MI versus TAU, Outcome 2 Reduce BZD > 50\%.

Review: Psychosocial interventions for benzodiazepine harmful use, abuse or dependence

Comparison: $2 \mathrm{Ml}$ versus TAU

Outcome: 2 Reduce BZD > 50\%

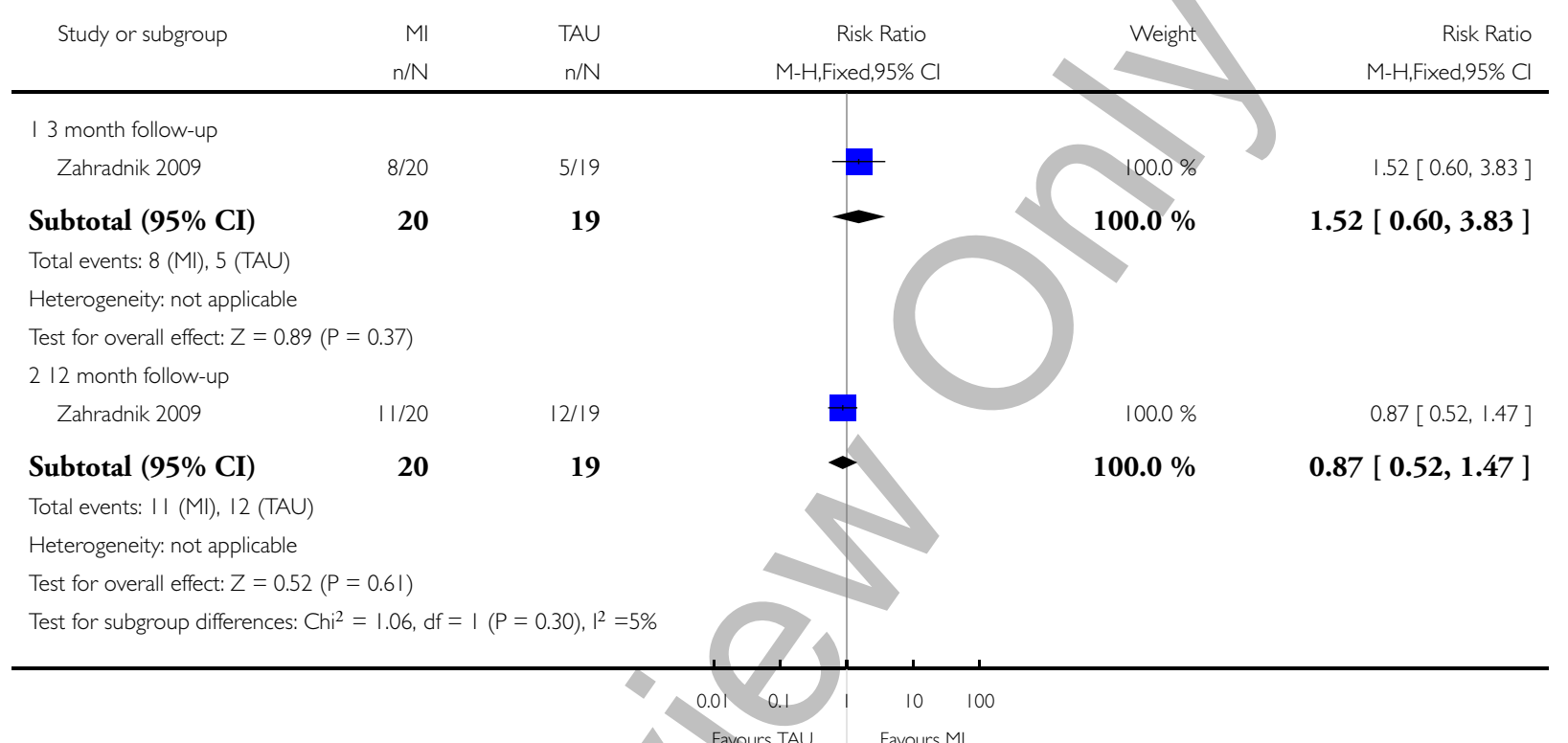




\section{Analysis 3.I. Comparison 3 Standardised interview (taper) versus TAU, Outcome I Successful discontinuation of BZDs.}

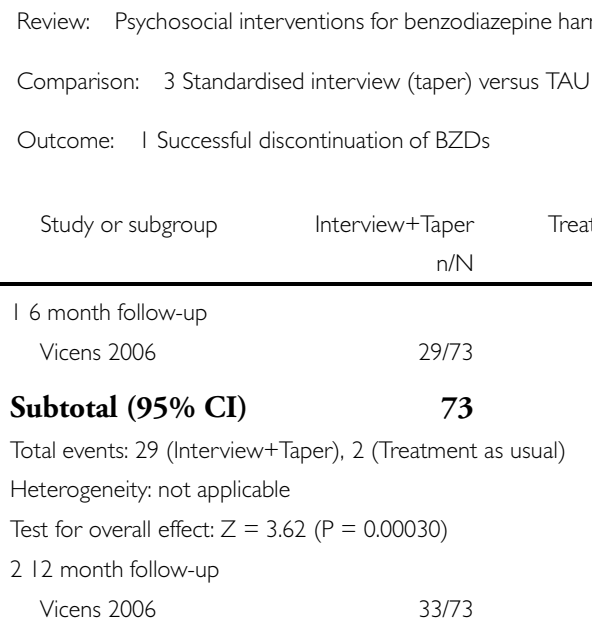

$29 / 73$

73

Total events: 29 (Interview+Taper), 2 (Treatment as usual) Heterogeneity: not applicable

Test for overall effect: $Z=3.62(P=0.00030)$

212 month follow-up$$
\text { Vicens } 2006
$$

73

Subtotal (95\% CI)

Total events: 33 (Interview+Taper), 6 (Treatment as usual) Heterogeneity: not applicable

Test for overall effect: $Z=3.91(P=0.000092)$

33 year follow-up

$$
\text { Vicens } 2006
$$

\section{Subtotal (95\% CI)}

Total events: 25 (Interview+Taper), 14 (Treatment as usual) Heterogeneity: not applicable

Test for overall effect: $Z=1.67(P=0.096)$

Test for subgroup differences: $\mathrm{Chi}^{2}=10.34, \mathrm{df}=2(\mathrm{P}=0.01), \mathrm{I}^{2}=81 \%$
Risk Ratio M-H,Fixed,95\% Cl

$$
14 / 66
$$

Risk Ratio $\mathrm{M}-\mathrm{H}$, Fixed,95\% Cl

|3.11 [3.25, 52.83$]$

13.11 [ 3.25, 52.83 ]
$4.97[2.23,11.11]$

$100.0 \%$

$4.97[2.23,11.11]$

$100.0 \%$

$1.61[0.92,2.84$ ] 
Analysis 3.2. Comparison 3 Standardised interview (taper) versus TAU, Outcome 2 Reduce BZD > 50\%.

Review: Psychosocial interventions for benzodiazepine harmful use, abuse or dependence

Comparison: 3 Standardised interview (taper) versus TAU

Outcome: 2 Reduce BZD > 50\%

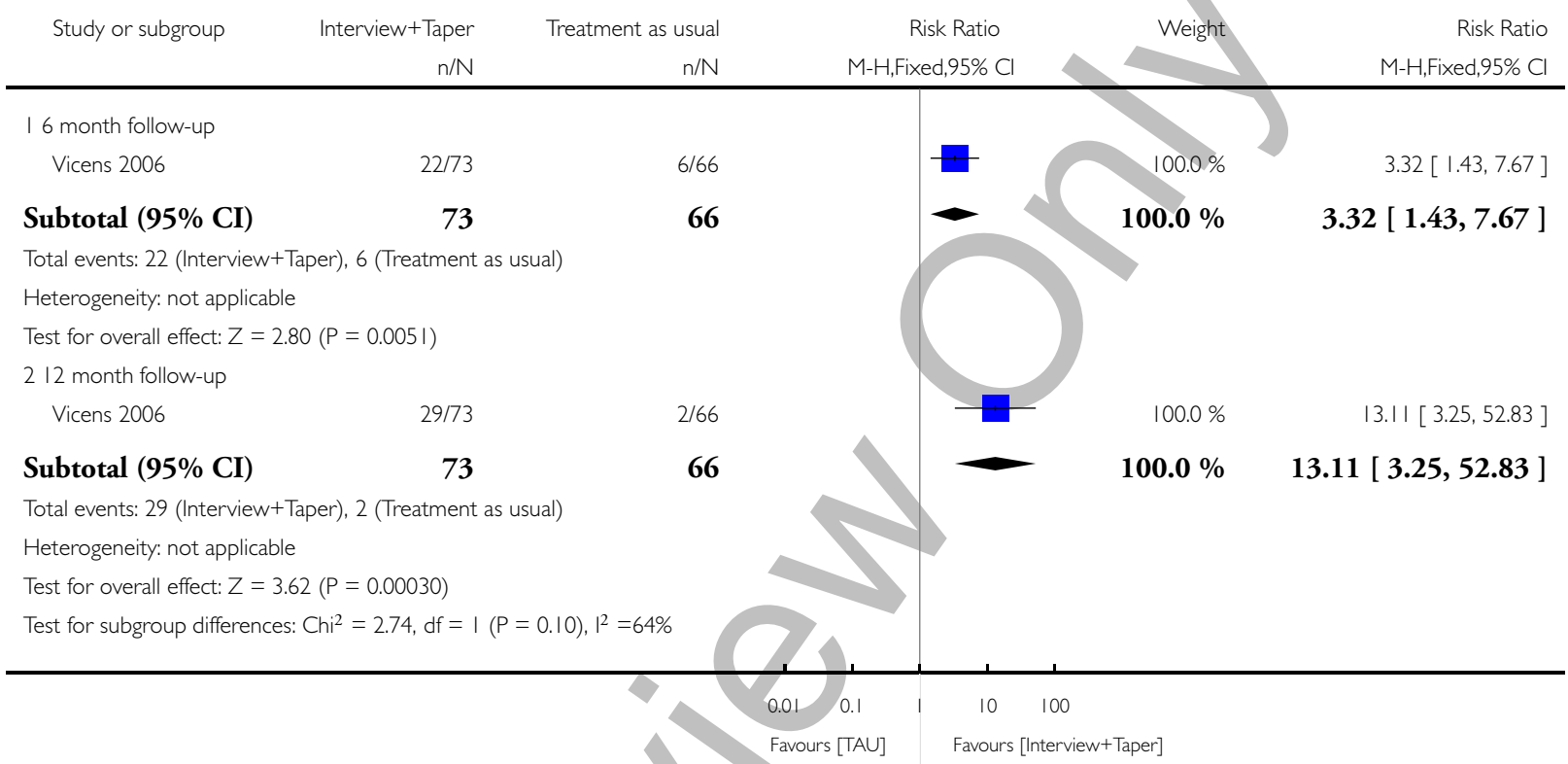




\section{Analysis 4.I. Comparison 4 CBT (no taper) versus TAU, Outcome I BZD positive urine rate.}

Review: Psychosocial interventions for benzodiazepine harmful use, abuse or dependence

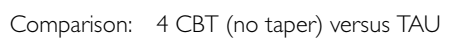

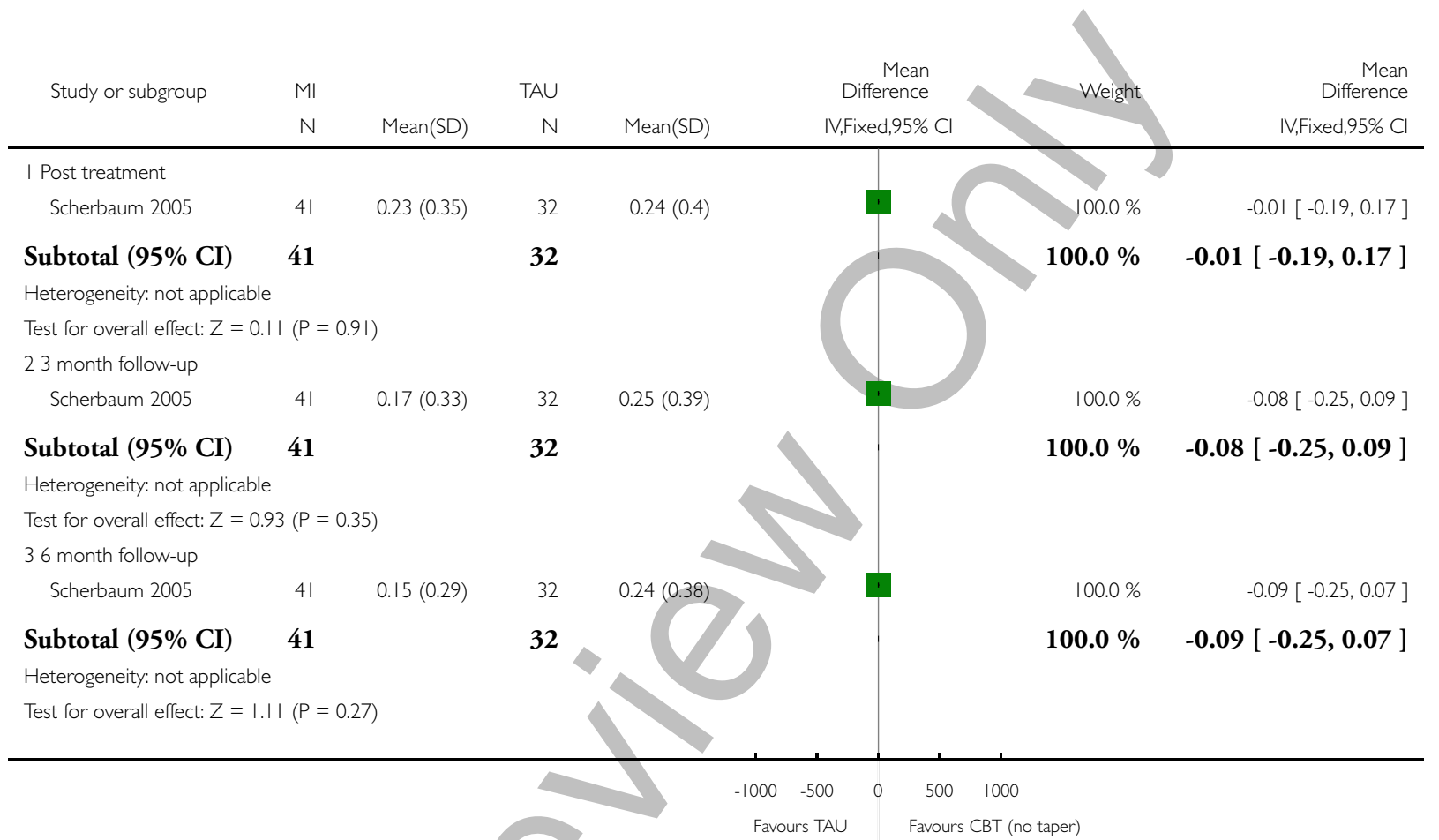


Analysis 5.I. Comparison 5 Self-help booklet plus CBT versus self-help booklet, Outcome I Change in OTI score for BZD use.

Review: Psychosocial interventions for benzodiazepine harmful use, abuse or dependence

Comparison: 5 Self-help booklet plus CBT versus self-help booklet

Outcome: I Change in OTI score for BZD use

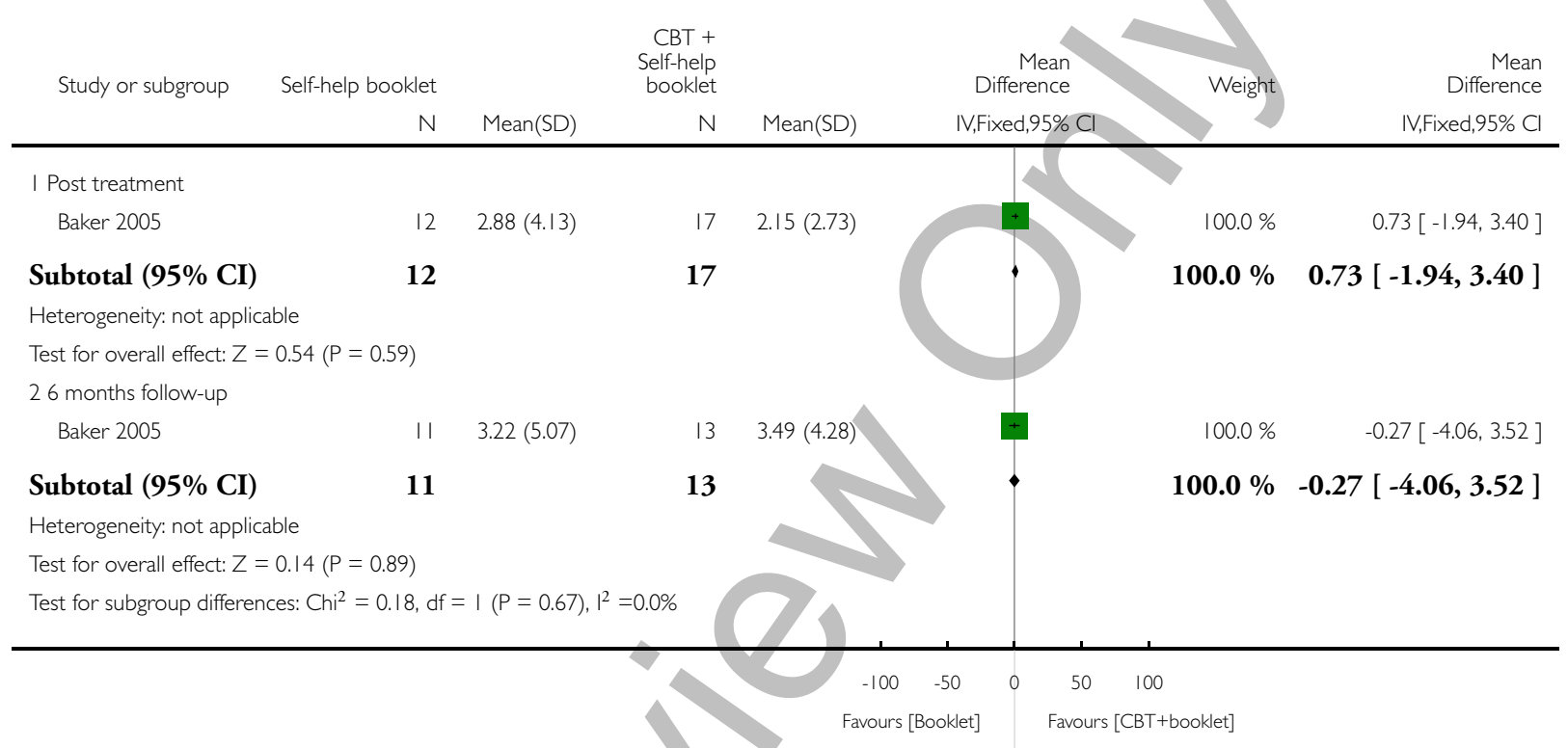

Psychosocial interventions for benzodiazepine harmful use, abuse or dependence (Review)

Copyright @ 2015 The Cochrane Collaboration. Published by John Wiley \& Sons, Ltd. 
Analysis 6.1. Comparison 6 Complaints management (additional relaxation) versus anxiety management (relaxation), Outcome I Successful discontinuation of BZDs.

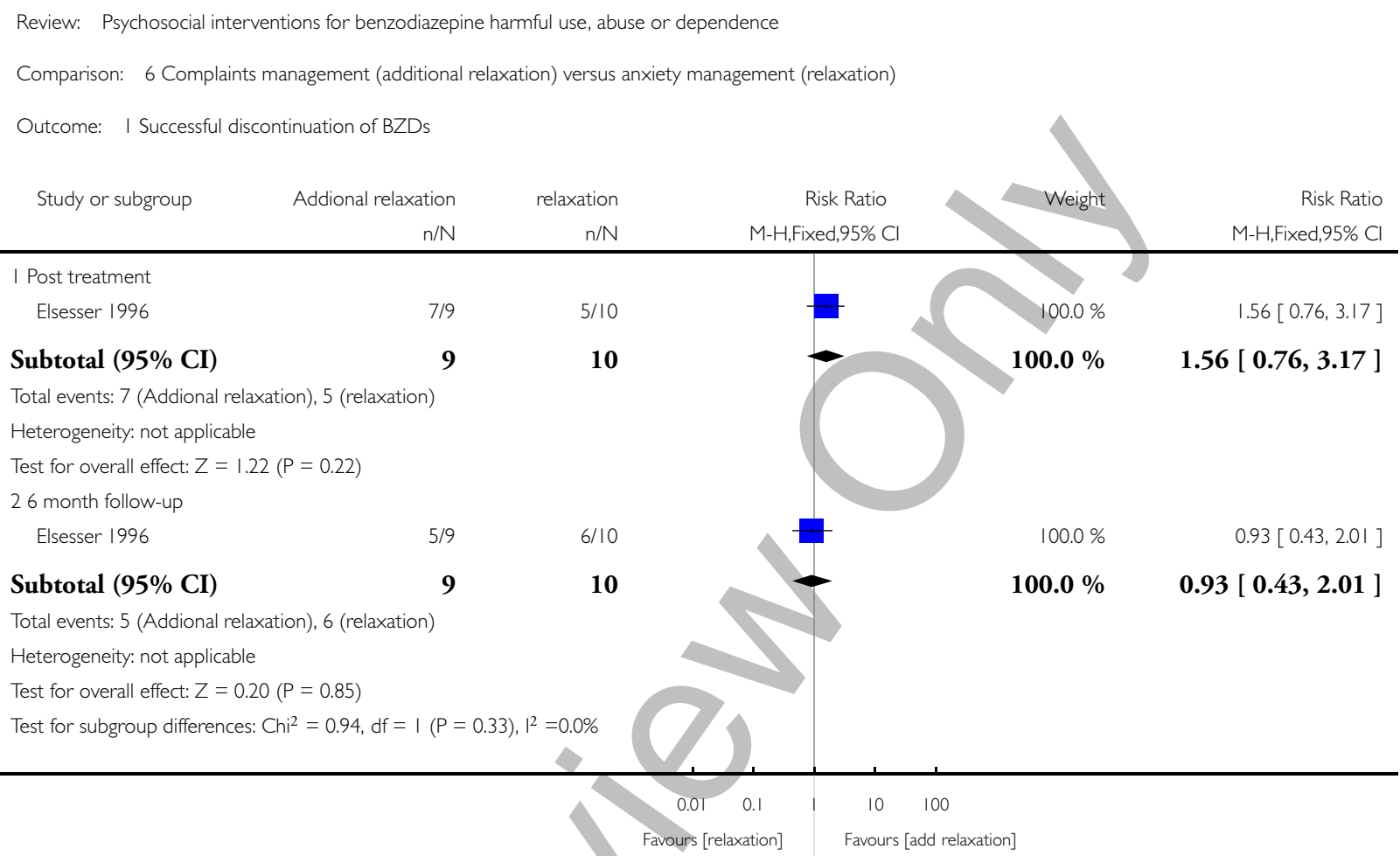




\section{Analysis 7.I. Comparison 7 Consultation (plus letter) versus TAU, Outcome I Successful discontinuation of BZD.}

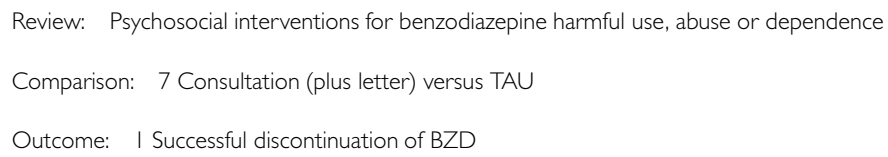

\section{Analysis 8.I. Comparison 8 E-counselling versus onsite counselling, Outcome I Positive BZD urine} toxicology.

Review: Psychosocial interventions for benzodiazepine harmful use, abuse or dependence

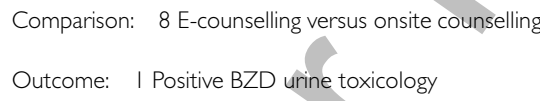

Heterogeneity: not applicable

Test for overall effect: $Z=0.46(P=0.64)$

Test for subgroup differences: Not applicable

$$
\begin{aligned}
& \begin{array}{lllll}
-1000 & -500 & 0 & 500 & 1000
\end{array} \\
& \text { Favours [onsite] Favours [e-counselling] }
\end{aligned}
$$


Analysis 9.I. Comparison 9 Relaxation versus TAU, Outcome I Successful discontinuation of BZDs.

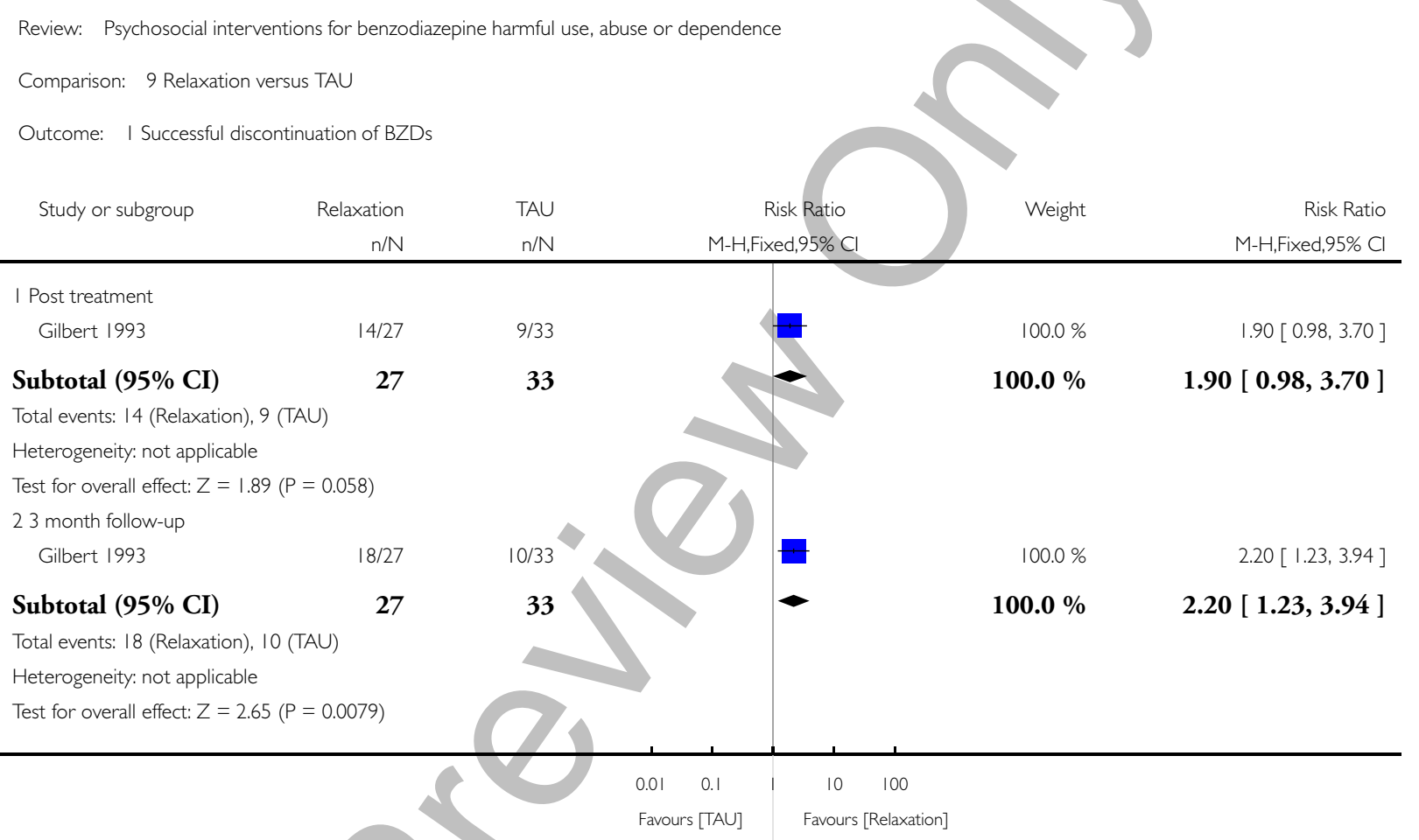


Analysis 10.I. Comparison IO Tailored letter versus GP letter, Outcome I Successful discontinuation of BZDs.

Review: Psychosocial interventions for benzodiazepine harmful use, abuse or dependence

Comparison: 10 Tailored letter versus GP letter

Outcome: I Successful discontinuation of BZDs

\begin{tabular}{|c|c|c|c|c|}
\hline Study or subgroup & $\begin{array}{r}\text { Tailored Letter } \\
\mathrm{n} / \mathrm{N}\end{array}$ & $\begin{array}{l}\text { Letter } \\
\mathrm{n} / \mathrm{N}\end{array}$ & $\begin{array}{c}\text { Risk Ratio } \\
\text { M-H,Fixed,95\% Cl }\end{array}$ & $\begin{array}{r}\text { Risk Ratio } \\
\text { M-H,Fixed,95\% Cl}\end{array}$ \\
\hline I 12 month follow-up & & & & \\
\hline Ten Wolde 2008 & $40 / 163$ & $23 / 159$ & & $1.70[1.07,2.70]$ \\
\hline
\end{tabular}

Subtotal (95\% CI)

163159

159

Total events: 40 (Tailored Letter), 23 (Letter)

Heterogeneity: not applicable

Test for overall effect: $Z=2.23(P=0.026)$

Test for subgroup differences: Not applicable 


\section{Analysis I I.I. Comparison I I Relaxation (plus taper) versus taper, Outcome I Successful discontinuation of BZDs.}

Review: Psychosocial interventions for benzodiazepine harmful use, abuse or dependence

Comparison: I| Relaxation (plus taper) versus taper

Outcome: I Successful discontinuation of BZDs

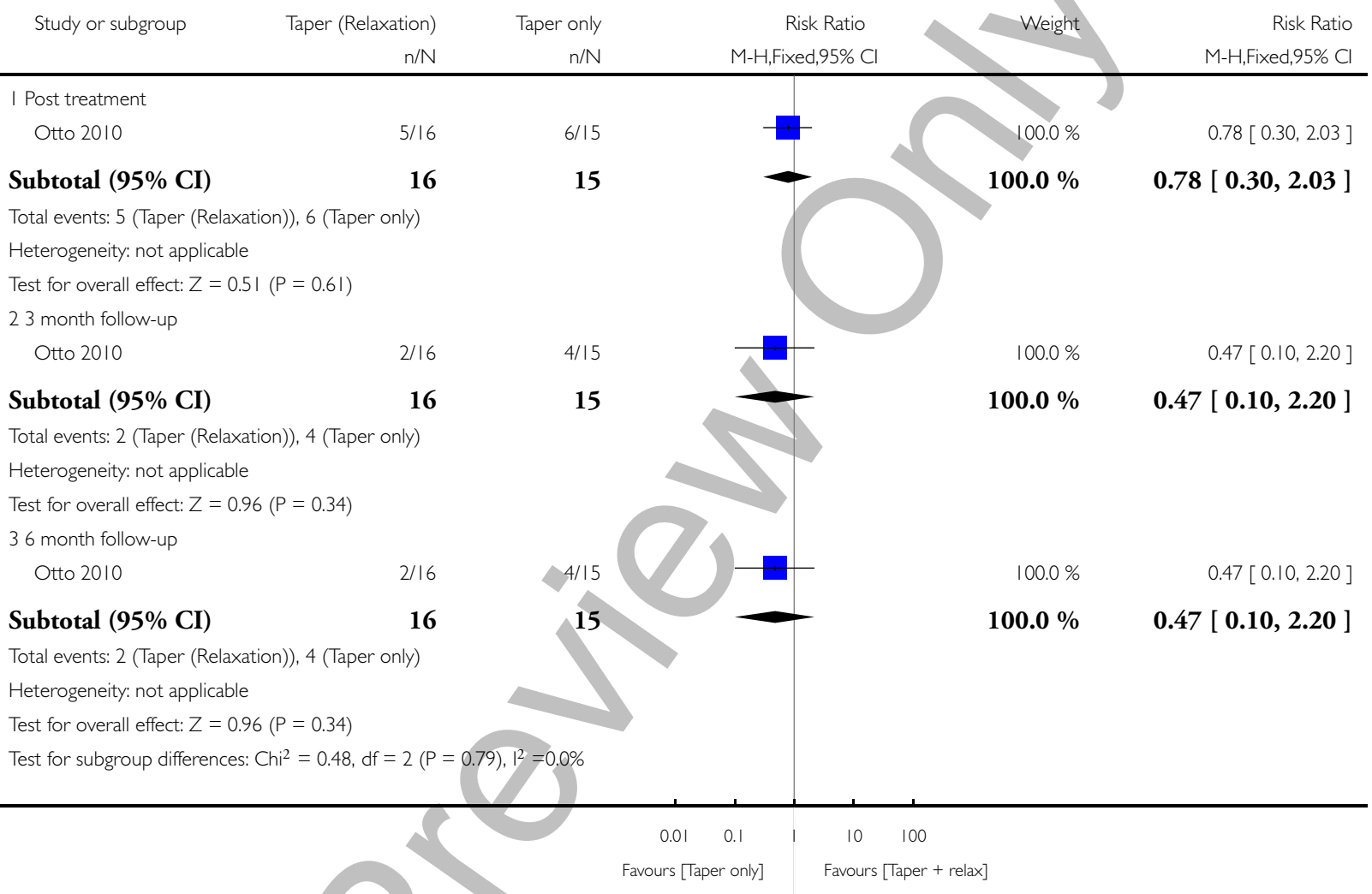




\section{Analysis 12.I. Comparison I2 Enhanced skills training (relaxation) versus limited skills training (relaxation), Outcome I Change in prescribed diazepam dose $(\mathrm{mg})$.}

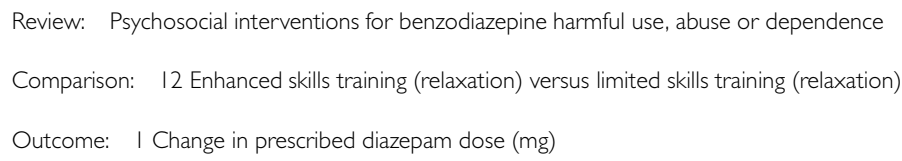

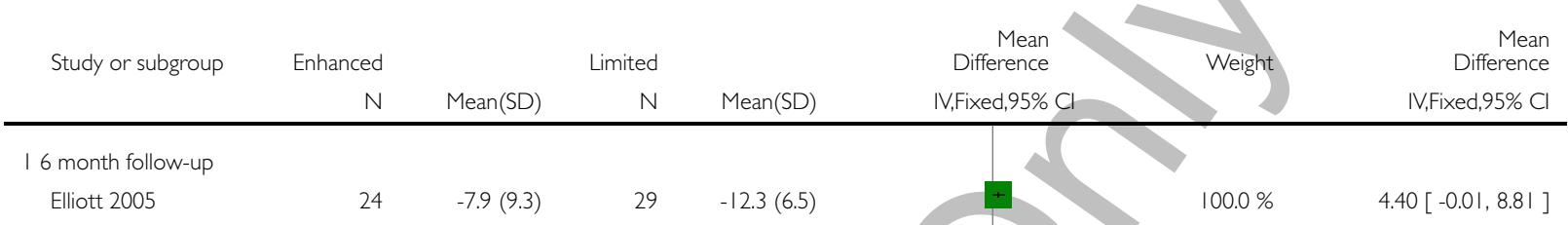

Subtotal (95\% CI) 24

Heterogeneity: not applicable

\section{APPENDICES}

\section{Appendix I. CENTRAL search strategy}

1. MeSH descriptor Substance-Related Disorders explode all trees

2. (abuse* OR abusing OR dependen* OR addict* OR misuse OR polyabuse OR overdose OR abstin* OR abstain OR withdrawal):ti,ab,kw

3. \#1 OR \#2

4. MeSH descriptor Benzodiazepines explode all trees

5. (benzodiazepine* OR BZD OR chlordiazepoxide OR diazepam OR alprazolam OR lorazepam OR prazepam OR clobazam OR bromazepam OR flurazepam OR triazolam OR clonazepam OR temazepam OR nitrazepam OR nitrazepam OR lormetazepam OR flunitrazepam):ti,ab,kw

6. MeSH descriptor: [Anti-Anxiety Agents] explode all trees

7. \#4 OR \#5 OR \#6

8. (psychotherap* OR incentive* OR voucher OR psychosocial* OR reinforcement OR motivation* OR contingent* OR advice OR biofeedback OR community OR education*):ti,ab,kw

9. (behavio* near/2 therap*):ti,ab

10. MeSH descriptor Psychotherapy explode all trees

11. MeSH descriptor Counseling explode all trees

12. (cognitive near/2 therapy):ti,ab

13. CBT:ti,ab

14. (brief near/2 intervention): $t i, a b$

15. (early near/2 intervention):ti,ab

Psychosocial interventions for benzodiazepine harmful use, abuse or dependence (Review)

Copyright @ 2015 The Cochrane Collaboration. Published by John Wiley \& Sons, Ltd. 
16. (family near/2 therapy):ti,ab

17. (coping near/2 skill*) ti,ab

18. "supportive expressive therapy"

19. (social near/2 skil $\left.{ }^{*}\right): \mathrm{ti}, \mathrm{ab}$

20. (stress near/2 management):ti,ab

21. MeSH descriptor: [Social Support ]explode all trees

22. MeSH descriptor: [Relaxation Therapy] explode all trees

23. "relapse prevention"

24. "dialectical behaviour"

25. (motivational near/2 interview*):ti,ab

26. (motivational near/2 enhance*):ti,ab

27. \#8 OR \#9 OR \#10 OR \#11 OR \#12 OR\#13 OR \#14 OR \#15 OR \#16 OR \#17 OR \#18 OR\#19 OR \#20 OR \#21 OR \#22

OR \#23 OR \#24 OR \#25 OR \#26

28. \#3 AND \#7 AND \#27

\section{Appendix 2. PubMed search strategy}

1. "Substance-Related Disorders" [Mesh]

2. abuse*[tiab] OR abusing[tiab] OR dependen*[tiab] OR addict*[tiab] OR misuse[tiab] OR polyabuse[tiab] OR overdose[tiab] OR abstin*[tiab] OR abstain[tiab] OR withdrawal[tiab]

3. \#1 OR \#2

4. "Benzodiazepines" [Mesh]

5. Benzodiazepine*[tiab] OR BZD[tiab] OR chlordiazepoxide[tiab] OR diazepam[tiab] OR alprazolam[tiab] OR lorazepam[tiab] OR prazepam[tiab] OR clobazam[tiab] OR bromazepam[tiab] OR flurazepam[tiab] OR triazolam[tiab] OR clonazepam[tiab] OR temazepam[tiab] OR nitrazepam[tiab] OR nitrazepam[tiab] OR lormetazepam[tiab] OR flunitrazepam[tiab]

6. "Anti-Anxiety Agents"[Mesh]

7. \#4 OR \#5 OR \#6

8. psychotherap*[tiab] OR incentive*[tiab] OR voucher[tiab] OR psychosocial*[tiab] OR reinforcement[tiab] OR motivation* [tiab] OR contingent*[tiab] OR advice[tiab] OR biofeedback[tiab] OR community[tiab] OR education*[tiab]

9. (behavio*[tiab] AND therap*[tiab])

10. Psychotherapy [Mesh]

11. Counseling[Mesh] OR counsel*[tiab]

12. cognitive therapy[tiab]

13. CBT[tiab]

14. brief intervention[tiab]

15. early intervention[tiab]

16. family therapy[tiab]

17. coping skill*[tiab]

18. supportive expressive therapy

19. social skill[tiab]

20. stress management[tiab]

21. "Social Support" [Mesh]

22. "Relaxation Therapy"[Mesh]

23. "relapse prevention"

24. "dialectical behaviour"

25. motivational interview*[tiab]

26. motivational enhance* $[$ tiab]

27. \#8 OR \#9 OR \#10 OR \#11 OR \#12 OR \#13 OR \#14 OR \#15 OR \#16 OR \#17 OR \#18 OR \#19 OR \#20 OR \#21 OR \#22 OR \#23 OR \#24 OR \#25 OR \#26

28. randomized controlled trial $[\mathrm{pt}]$

29. controlled clinical trial $[\mathrm{pt}]$

30. randomized [tiab]

Psychosocial interventions for benzodiazepine harmful use, abuse or dependence (Review)

Copyright @ 2015 The Cochrane Collaboration. Published by John Wiley \& Sons, Ltd. 
31. placebo [tiab]

32. drug therapy $[\mathrm{sh}]$

33. randomly [tiab]

34. trial [tiab]

35. groups [tiab]

36. animals $[\mathrm{mh}]$ NOT humans $[\mathrm{mh}]$

37. \#28 OR \#29 OR \#30 OR \#31 OR \#32 OR \#33 OR \#34 OR \#35

38. \#37 NOT \#36

39. \#3 AND \#7 AND \#27 AND \#38

\section{Appendix 3. EMBASE search strategy}

1. substance AND related AND 'disorder'/exp

2. abuse*:ab,ti OR abusing:ab,ti OR dependen*:ab,ti OR addict*:ab,ti OR misuse:ab,ti OR polyabuse:ab,ti OR overdose:ab,ti OR abstin*:ab,ti OR withdrawal:ab,ti

3. \#1 OR \#2

4. 'benzodiazepines'/exp

5. benzodiazepine*:ab,ti OR bzd:ab,ti OR chlordiazepoxide:ab,ti OR diazepam:ab,ti OR alprazolam:ab,ti OR lorazepam:ab,ti OR prazepam:ab,ti OR clobazam:ab,ti OR bromazepam:ab,ti OR flurazepam:ab,ti OR triazolam:ab,ti OR clonazepam:ab,ti OR temazepam:ab,ti OR nitrazepam:ab,ti OR lormetazepam:ab,ti OR flunitrazepam:ab,ti

6. anti AND 'anxiety'/exp AND agents

7. \#4 OR \#5 OR \#6

8. psychotherap*:ab,ti OR incentive*:ab,ti OR voucher:ab,ti OR psychosocial*:ab,ti OR reinforcement:ab,ti OR motivation*:ab,ti OR contingent*:ab,ti OR advice:ab,ti OR biofeedback:ab,ti OR community:ab,ti OR education*:ab,ti

9. behavio*:ab,ti AND therap*:ab,ti

10. 'psychotherapy'/exp

11. 'counseling'/exp OR counsel*:ab,ti

12. cognitive AND therapy:ab,ti

13. cbt:ab,ti

14. brief AND intervention:ab,ti

15. early AND intervention:ab,ti

16. 'family'/exp AND therapy:ab,ti

17. 'coping'/exp AND skill*:ab,ti

18. supportive AND expressive AND therapy'/exp

19. social AND skill:ab,ti

20. 'stress'/exp AND management:ab,ti

21. social AND support

22. 'relaxation'/exp AND 'therapy'/exp

23. 'relapse'/exp AND 'prevention'/exp

24. dialectical AND behav*

25. motivational AND interview*:ab,ti

26. motivational AND enhance*:ab,ti

27. \#8 OR \#9 OR \#10 OR \#11 OR \#12 OR \#13 OR \#14 OR \#15 OR \#16 OR \#17 OR \#18 OR \#19 OR \#20 OR \#21 OR \#22 OR \#23 OR \#24 OR \#25 OR \#26

28. randomized AND controlled AND trial

29. controlled AND clinical AND trial

30. randomized:ab,ti

31. placebo:ab,ti

32. 'drug'/exp AND therapy:Ink

33. randomly:ab,ti

34. trial:ab,ti

35. groups:ab,ti

Psychosocial interventions for benzodiazepine harmful use, abuse or dependence (Review)

Copyright $\odot 2015$ The Cochrane Collaboration. Published by John Wiley \& Sons, Ltd. 
36. animals:de NOT humans:de

37. \#28 OR \#29 OR\#30 OR \#31 OR\#32 OR\#33 OR\#34 OR \#35

38. \#37 NOT \#36

39. \#3 AND \#7 AND \#27 AND \#38

\section{Appendix 4. CINAHL search strategy}

1. MH "Substance Use Disorders+"

2. TI(addict* or overdos* or intoxicat* or abstin* or abstain or withdraw* or abus* or abusing or misus* or disorder* or dependen* or polyabus*)

3. AB (addict* or overdos* or intoxicat* or abstin* or abstain or withdraw* or abus* or abusing or misus* or disorder* or dependen* or polyabus*)
4. $\mathrm{S} 1$ or $\mathrm{S} 2$ or $\mathrm{S} 3$

5. MH "Antianxiety Agents, Benzodiazepine"

6. TI benzodiazepine* or TI bzd or TI chlordiazepoxide or TI diazepam or TI alprazolam or TI lorazepam or TI prazepam or TI clobazam or TI bromazepam or TI flurazepam or TI triazolam or TI clonazepam

7. $\mathrm{AB}$ benzodiazepine* or $\mathrm{AB}$ bzd or $\mathrm{AB}$ chlordiazepoxide or $\mathrm{AB}$ diazepam or $\mathrm{AB}$ alprazolam or $\mathrm{AB}$ lorazepam or $\mathrm{AB}$ prazepam or $\mathrm{AB}$ clobazam or $\mathrm{AB}$ bromazepam or $\mathrm{AB}$ flurazepam or $\mathrm{AB}$ triazolam or $\mathrm{AB}$ clonazepam

8. TI temazepam or TI nitrazepam or TI lormetazepam or TI flunitrazepam

9. $\mathrm{AB}$ temazepam or $\mathrm{AB}$ nitrazepam or $\mathrm{AB}$ lormetazepam or $\mathrm{AB}$ flunitrazepam

10. $\mathrm{MH}$ "Antianxiety Agents"

11. $\mathrm{S} 5$ or $\mathrm{S} 6$ or $\mathrm{S} 7$ or $\mathrm{S} 8$ or $\mathrm{S} 9$ or $\mathrm{S} 10$

12. $S 4$ and $S 11$

13. TI psychotherap* or TI incentive* or TI voucher or TI psychosocial* or TI reinforcement or TI motivation* or TI contingent* or TI advice or TI biofeedback or TI community or TI education*

14. $\mathrm{AB}$ psychotherap* or $\mathrm{AB}$ incentive* or $\mathrm{AB}$ voucher or $\mathrm{AB}$ psychosocial* or $\mathrm{AB}$ reinforcement or $\mathrm{AB}$ motivation* or $\mathrm{AB}$ contingent* or $\mathrm{AB}$ advice or $\mathrm{AB}$ biofeedback or $\mathrm{AB}$ community or $\mathrm{AB}$ education*

15. TI(behavio* $\mathrm{N} 3$ therap $\left.^{*}\right)$ or $\mathrm{AB}\left(\right.$ behavio* $^{*} \mathrm{~N} 3$ therap*)

16. (MH "Psychotherapy")

17. (MH "Counseling")

18. TI counsel* or $\mathrm{AB}$ counsel*

19. TI (cognitive $\mathrm{N} 2$ therap*) or $\mathrm{AB}$ (cognitive $\mathrm{N} 2$ therap*) or TI (family $\mathrm{N} 1$ therap*) or $\mathrm{AB}$ (family $\mathrm{N} 1$ therap*)

20. TI (brief $\mathrm{N} 3$ intervention*) or $\mathrm{AB}$ (brief $\mathrm{N} 3$ intervention) or TI (early N3 intervention*) or $\mathrm{AB}$ (early N3 intervention)

21. TI (coping N1 skill*) or AB (coping N1 skill*) or TI (social N1 skill*) or AB (social N1 skill*)

22. "supportive expressive therapy"

23. TI"stress management" or AB"stress management"

24. TI (relapse N3 prevent*) or AB (relapse N3 prevent*)

25. $S 13$ or $S 14$ or $S 15$ or $S 16$ or $S 17$ or $S 18$ or $S 19$ or $S 20$ or $S 21$ or S22 or S23 or S24

26. MH "Clinical Trialst"

27. PT Clinical trial

28. TI clinic* $\mathrm{N} 1$ trial$^{*}$ or $\mathrm{AB}$ clinic $^{*} \mathrm{~N} 1$ trial $^{*}$

29. TI (singl* or doubl* or trebl* or tripl*) and TI (blind* or mask*)

30. $\mathrm{AB}$ (singl ${ }^{*}$ or doubl $^{*}$ or trebl* or tripl $\left.{ }^{*}\right)$ and $\mathrm{AB}\left(\right.$ blind $^{*}$ or mask $\left.{ }^{*}\right)$

31. TI randomi?ed control* trial $^{*}$ or $\mathrm{AB}$ randomi?ed control ${ }^{*}$ trial $^{*}$

32. MH "Random Assignment"

33. TI random* allocat* or AB random* allocat*

34. $\mathrm{MH}$ "Placebos"

35. TI placebo* or AB placebo*

36. MH "Quantitative Studies"

37. $\mathrm{S} 26$ or $\mathrm{S} 27$ or $\mathrm{S} 28$ or $\mathrm{S} 29$ or $\mathrm{S} 30$ or $\mathrm{S} 31$ or $\mathrm{S} 32$ or $\mathrm{S} 33$ or $\mathrm{S} 34$ or $\mathrm{S} 35$ or $\mathrm{S} 36$

38. $S 12$ and S25 and S37

Psychosocial interventions for benzodiazepine harmful use, abuse or dependence (Review) 


\section{Appendix 5. PsychINFO search strategy}

1. MH "Substance Use Disorders+"

2. TI(addict* or overdos* or intoxicat* or abstin* or abstain or withdraw* or abus* or abusing or misus* or disorder* or dependen* or polyabus*)

3. $\mathrm{AB}$ (addict* ${ }^{*}$ or overdos* or intoxicat* or abstin* or abstain or withdraw* or abus* or abusing or misus* or disorder* ${ }^{*}$ or dependen* $^{*}$ or polyabus*)

4. $\mathrm{S} 1$ or $\mathrm{S} 2$ or $\mathrm{S} 3$

5. MH "Antianxiety Agents, Benzodiazepine"

6. TI benzodiazepine* or TI bzd or TI chlordiazepoxide or TI diazepam or TI alprazolam or TI lorazepam or TI prazepam or TI clobazam or TI bromazepam or TI flurazepam or TI triazolam or TI clonazepam

7. $\mathrm{AB}$ benzodiazepine* or $\mathrm{AB}$ bzd or $\mathrm{AB}$ chlordiazepoxide or $\mathrm{AB}$ diazepam or $\mathrm{AB}$ alprazolam or $\mathrm{AB}$ lorazepam or $\mathrm{AB}$ prazepam or $\mathrm{AB}$ clobazam or $\mathrm{AB}$ bromazepam or $\mathrm{AB}$ flurazepam or $\mathrm{AB}$ triazolam or $\mathrm{AB}$ clonazepam

8. TI temazepam or TI nitrazepam or TI lormetazepam or TI flunitrazepam

9. $\mathrm{AB}$ temazepam or $\mathrm{AB}$ nitrazepam or $\mathrm{AB}$ lormetazepam or $\mathrm{AB}$ flunitrazepam

10. $\mathrm{MH}$ "Antianxiety Agents"

11. $\mathrm{S} 5$ or $\mathrm{S} 6$ or $\mathrm{S} 7$ or $\mathrm{S} 8$ or $\mathrm{S} 9$ or $\mathrm{S} 10$

12. $S 4$ and $S 11$

13. TI psychotherap* or TI incentive* or TI voucher or TI psychosocial* or TI reinforcement or TI motivation* or TI contingent* or TI advice or TI biofeedback or TI community or TI education*

14. $\mathrm{AB}$ psychotherap* or $\mathrm{AB}$ incentive* or $\mathrm{AB}$ voucher or $\mathrm{AB}$ psychosocial* or $\mathrm{AB}$ reinforcement or $\mathrm{AB}$ motivation* or $\mathrm{AB}$ contingent* or $\mathrm{AB}$ advice or $\mathrm{AB}$ biofeedback or $\mathrm{AB}$ community or $\mathrm{AB}$ education*

15. TI(behavio* N3 therap*) or $\mathrm{AB}\left(\right.$ behavio* $^{*} 3$ therap*)

16. (MH "Psychotherapy")

17. (MH "Counseling")

18. TI counsel* or $\mathrm{AB}$ counsel*

19. TI (cognitive N2 therap*) or AB (cognitive N2 therap*) or TI (family N1 therap*) or AB (family N1 therap*)

20. TI (brief $\mathrm{N} 3$ intervention*) or $\mathrm{AB}$ (brief N3 intervention) or TI (early N3 intervention*) or $\mathrm{AB}$ (early N3 intervention)

21. TI (coping N1 skill*) or AB (coping N1 skill*) or TI (social N1 skill*) or AB (social N1 skill*)

22. "supportive expressive therapy"

23. TI"stress management" or AB" stress management"

24. TI (relapse N3 prevent*) or AB (relapse N3 prevent*)

25. $S 13$ orS 14 or $S 15$ or $S 16$ or $S 17$ or $S 18$ or $S 19$ or $S 20$ or S21 or S22 or S23 or S24

26. MH "Clinical Trials+"

27. PT Clinical trial

28. TI clinic* $\mathrm{N} 1$ trial $^{*}$ or $\mathrm{AB}$ clinic $^{*} \mathrm{~N} 1$ trial$^{*}$

29. TI (singl* ${ }^{*}$ or doubl* or trebl* or tripl*) and TI (blind* or mask $\left.{ }^{*}\right)$

30. $\mathrm{AB}\left(\right.$ singl $^{*}$ or doubl* or trebl* or tripl $\left.{ }^{*}\right)$ and $\mathrm{AB}\left(\mathrm{blind}^{*}\right.$ or mask $\left.{ }^{*}\right)$

31. TI randomi?ed control* ${ }^{*}$ trial $^{*}$ or $\mathrm{AB}$ randomi?ed control* trial $^{*}$

32. $\mathrm{MH}$ "Random Assignment"

33. TI random* allocat* or AB random* allocat*

34. MH "Placebos"

35. TI placebo* or AB placebo*

36. MH "Quantitative Studies"

37. S26 or S27 or S28 or S29 or S30 or S31 or S32 or S33 or S34 or S35 or S36

38. $S 12$ and $S 25$ and S37

Psychosocial interventions for benzodiazepine harmful use, abuse or dependence (Review) 


\section{Appendix 6. ERIC search strategy}

1. all(benzodiazepine* $)$ OR all((chlordiazepoxide OR diazepam)) OR all((alprazolam OR lorazepam)) OR all((prazepam OR clobazam)) OR all((bromazepam OR flurazepam)) OR all((triazolam OR clonazepam)) OR all((temazepam OR nitrazepam)) OR all((lormetazepam OR flunitrazepam)) OR all(“Antianxiety Agents”)

2. all(addict* ${ }^{*}$ OR all((overdos* OR intoxicat*)) OR all((abstin* OR abstain)) OR all((withdraw* OR abus*)) OR all((abusing OR misus*)) OR all((disorder* OR dependen*)) OR all(polyabus*)

3. S1 AND S2

\section{Appendix 7. OVID search strategy}

1. substance related disorders.de.

2. (abuse* or abusing or dependen* or addict* or misuse or polyabuse or overdose or abstin* or abstain or withdrawal).ab,de,ti.

3. 1 or 2

4. benzodiazepines.de.

5. (Benzodiazepine* or BZD or chlordiazepoxide or diazepam or alprazolam or lorazepam or prazepam or clobazam or bromazepam or flurazepam or triazolam or clonazepam or temazepam or nitrazepam or nitrazepam or lormetazepam or flunitrazepam).ab,ti.

6. anti anxiety agents.de,ti.

7. 4 or 5 or 6

8. (psychotherap* or incentive* or voucher or psychosocial* or reinforcement or motivation* or contingent* or advice or biofeedback or community or education*).ab,ti.

9. (behavio* and therap*).ab,ti.

10. Psychotherapy.de.

11. Counseling.de.

12. "counsel*".ab,ti.

13. 11 or 12

14. cognitive therapy.ab,ti.

15. CBT.ab,ti.

16. brief intervention.ab,ti.

17. early intervention.ab,ti.

18. family therapy.ab,ti.

19. "coping skill*".ab,ti.

20. supportive expressive therapy.af.

21. social skill.ab,ti.

22. stress management.ab,ti.

23. Social Support.de.

24. Relaxation Therapy.de.

25. relapse prevention.af.

26. dialectical behaviour.af.

27. "motivational interview".ab,ti.

28. "motivational enhance*".ab,ti.

29. 8 or 9 or 10 or 13 or 14 or 15 or 16 or 17 or 18 or 19 or 20 or 21 or 22 or 23 or 24 or 25 or 26 or 27 or 28

30. randomized controlled trial.pt.

31. controlled clinical trial.pt.

32. randomized.ab,ti.

33. placebo.ab,ti.

34. drug therapy.de.

35. randomly.ab,ti.

36. trial.ab,ti.

37. groups.ab,ti.

38. quantitative studies.de.

39. 30 or 31 or 32 or 33 or 34 or 35 or 36 or 37 or 38

40. 3 and 7 and 29 and 39

Psychosocial interventions for benzodiazepine harmful use, abuse or dependence (Review)

Copyright () 2015 The Cochrane Collaboration. Published by John Wiley \& Sons, Ltd. 


\section{Appendix 8. AMED Allied and Complementary Medicine search strategy}

1. TX Substance-related

2. TI addict* or overdos* or intoxicat* or abstin* or abstain or withdraw* or abus* or abusing or misus* ${ }^{*}$ or disorder* $^{*}$ or dependen* $^{*}$ or polyabus*

3. AB addict* or overdos* or intoxicat* or abstin* or abstain or withdraw* or abus* or abusing or misus* or disorder* or dependen* or polyabus*

4. $\mathrm{S} 1$ or $\mathrm{S} 2$ or $\mathrm{S} 3$

5. TX Antianxiety Agents

6. TX Benzoic

7. (TX Benzoic) AND (S5 or S6)

8. TI benzodiazepine* or bzd or chlordiazepoxide or diazepam or alprazolam or lorazepam or prazepam or clobazam or bromazepam or flurazepam or triazolam or clonazepam or temazepam or nitrazepam or lormetazepam or flunitrazepam

9. $\mathrm{AB}$ benzodiazepine* or bzd or chlordiazepoxide or diazepam or alprazolam or lorazepam or prazepam or clobazam or bromazepam or flurazepam or triazolam or clonazepam or temazepam or nitrazepam or lormetazepam or flunitrazepam

10. (AB ( benzodiazepine* or bzd or chlordiazepoxide or diazepam or alprazolam or lorazepam or prazepam or clobazam or bromazepam or flurazepam or triazolam or clonazepam or temazepam or nitrazepam or lormetazepam or flunitrazepam )) AND (S7 or S8 or S9)

11. S4 and S10

12. TI psychotherap* or incentive* or voucher or psychosocial* or reinforcement or motivation* or contingent* or advice or biofeedback or community or education*

13. $\mathrm{AB}$ psychotherap* or incentive* or voucher or psychosocial* or reinforcement or motivation* or contingent* or advice or biofeedback or community or education*

14. AB (behavio* therap*) OR TI (behavio* ${ }^{*}$ therap*)

15. TX Psychotherapy

16. TX Counseling

17. TI counsel* OR AB counsel*

18. TI cognitive therap* OR AB cognitive therap* OR TI family therap* OR AB family therap* OR TI brief intervention* OR AB brief intervention* OR TI early intervention* OR AB early intervention* OR TI coping skill* OR AB coping skill* OR TI social skill* OR AB social skill*

19. TX supportive expressive therapy

20. TI stress management OR AB stress management OR TI relapse prevent* OR AB relapse prevent*

21. $\mathrm{S} 12$ or $\mathrm{S} 13$ or $\mathrm{S} 14$ or $\mathrm{S} 15$ or $\mathrm{S} 16$ or $\mathrm{S} 17$ or $\mathrm{S} 18$ or $\mathrm{S} 19$ or $\mathrm{S} 20$

22. TX clinical trials

23. TI clinic* OR AB clinic* OR TI trial* OR AB trial*

24. TI singl* OR TI doubl OR TI trebl* OR TI tripl*

25. TI blind* OR TI mask

26. (TI blind* OR TI mask) AND (S24 and S25)

27. $\mathrm{AB}$ singl* $\mathrm{OR}$ AB doubl* OR AB trebl* OR AB tripl*

28. AB blind* OR AB mask*

29. S27 and S28

30. TI randomi?ed control* trial $^{*} \mathrm{OR}$ AB randomi?ed control* trial $^{*}$

31. TX random assignment

32. TI random* allocat* OR AB random* allocat*

33. TX Placebos

34. TI Placebo* OR AB placebo*

35. TX Quantitative studies

36. $\mathrm{S} 22$ or $\mathrm{S} 26$ or $\mathrm{S} 29$ or $\mathrm{S} 30$ or $\mathrm{S} 31$ or $\mathrm{S} 32$ or $\mathrm{S} 33$ or $\mathrm{S} 34$ or $\mathrm{S} 35$

37. $S 11$ and S21 and S36

Psychosocial interventions for benzodiazepine harmful use, abuse or dependence (Review)

Copyright $\odot 2015$ The Cochrane Collaboration. Published by John Wiley \& Sons, Ltd. 


\section{Appendix 9. ASSIA Applied Social Sciences Index \& Abstracts}

1. S1 su(substance use)

2. S2 ti(abuse* OR abusing OR dependen* OR addict* OR misuse OR polyabuse OR overdose OR abstin* OR abstain OR withdrawal)

3. S3 ab(abuse* OR abusing OR dependen* OR addict* OR misuse OR polyabuse OR overdose OR abstin* OR abstain OR withdrawal)

4. S4 S1 OR S2 OR S3

5. $\mathrm{S} 5 \mathrm{su}$ (benzodiazepines)

6. S6 ti(epam[tiab] OR temazepam[tiab] OR nitrazepam[tiab] OR nitrazepam[tiab] OR lormetazepam[tiab] OBenzodiazepine* OR BZD OR chlordiazepoxide OR diazepam OR alprazolam OR lorazepam OR prazepam OR clobazam OR bromazepam OR flurazepam OR triazolam OR clonazepam OR temazepam OR nitrazepam OR nitrazepam OR lormetazepam OR flunitrazepam)

7. S7 ab(epam[tiab] OR temazepam[tiab] OR nitrazepam[tiab] OR nitrazepam[tiab] OR lormetazepam[tiab] OBenzodiazepine* OR BZD OR chlordiazepoxide OR diazepam OR alprazolam OR lorazepam OR prazepam OR clobazam OR bromazepam OR flurazepam OR triazolam OR clonazepam OR temazepam OR nitrazepam OR nitrazepam OR lormetazepam OR flunitrazepam)

8. S8 S5 OR S6 OR S7 S9 ti(psychotherap* OR incentive* OR voucher OR psychosocial* OR reinforcement OR motivation* OR contingent* OR advice OR biofeedback OR community OR education*) S10 ab(psychotherap* OR incentive* OR voucher OR psychosocial* OR reinforcement OR motivation* OR contingent* OR advice OR biofeedback OR community OR education*)

9. S9 ti(behavio* AND therap*)

10. S10 ab(behavio* AND therap*)

11. S11 su(psychotherapy)

12. $S 12$ su(counselling) OR ti(counsel*) OR ab(counsel*)

13. $S 13$ ti(cognitive therapy)

14. S14 ab(cognitive therapy)

15. $\mathrm{S} 15 \mathrm{ab}(\mathrm{CBT})$

16. $\mathrm{S} 16 \mathrm{ti}(\mathrm{CBT})$

17. $\mathrm{S} 17$ ti(brief intervention)

18. $\mathrm{S} 18 \mathrm{ab}$ (brief intervention)

19. S19 ti(early intervention)

20. S20 ab(early intervention)

21. S21 ti(family therapy)

22. $S 22$ ab(family therapy)

23. S23 ti(coping skill*)

24. S24 ab(coping skill ${ }^{*}$ )

25. S25 su(supportive expressive therapy)

26. S26 ti(social skill)

27. S27 ab(social skill)

28. S28 ti(stress management)

29. $S 29$ ab(stress management)

30. S30 su(social support)

31. S31 su(relaxation therapy)

32. S32 all(relapse prevention)

33. S33 all(dialectical behaviour)

34. S34 ti(motivational interview*)

35. S35 ab(motivational interview*)

36. $S 36$ ti(motivational enhance*)

37. S37 ab(motivational enhance*)

38. S38 S9 OR S 10 OR S 11 OR S 12 OR S 13 OR S 14 OR S 15 OR S 16 OR S 17 OR S 18 OR S 19 OR S 20 OR S 21 OR S 22 OR S23 OR S24 OR S25 OR S26 OR S27 OR S28 OR S29 OR S 30 OR S 31 OR S 32 OR S 33 OR S34 OR S 35 OR S 36 OR S 37 OR S38 OR S39

39. $\mathrm{S} 39 \mathrm{su}$ (randomized controlled trial)

40. $\mathrm{S} 40$ all(controlled clinical trial)

41. $\mathrm{S} 41$ ti(randomized)

Psychosocial interventions for benzodiazepine harmful use, abuse or dependence (Review)

Copyright @ 2015 The Cochrane Collaboration. Published by John Wiley \& Sons, Ltd. 
42. $\$ 42 \mathrm{ab}$ (randomized)

43. $S 43$ ti(placebo)

44. $\$ 44 \mathrm{ab}$ (placebo)

45. $\mathrm{S} 45 \mathrm{su}$ (drug therapy)

46. $S 46$ ti(randomly)

47. $\mathrm{S} 47 \mathrm{ab}$ (randomly)

48. $S 48$ ti(trial)

49. $\$ 49 \mathrm{ab}$ (trial)

50. $\mathrm{S} 50$ ti(groups)

51. S51 ab(groups)

52. S52 all(animals) NOT all(humans)

53. S53 S41 OR S42 OR S43 OR S44 OR S 45 OR S46 OR S 47 OR S 48 OR S49 OR S50 OR S51 OR S52 OR S53 S56 S55 NOT 554

54. S54 S4 AND S8 AND S40 AND S56

\section{Appendix 10. LILACS search strategy}

benzodiazepines (limited to humans)

\section{Appendix I I. Web of Science search strategy}

1. $\mathrm{TS}=$ Substance Use Disorders

2. TS $=$ (abuse* OR abusing OR dependen* OR addict* OR misuse OR polyabuse OR overdose OR abstin* OR abstain OR withdrawal)

3. \#2 OR \#1

4. $\mathrm{TS}=$ benzodiazepine

5. $\mathrm{TS}=$ (Benzodiazepine OR BZD OR chlordiazepoxide OR diazepam OR alprazolam OR lorazepam OR prazepam OR clobazam OR bromazepam OR flurazepam OR triazolam OR clonazepam OR temazepam OR nitrazepam OR nitrazepam OR lormetazepam OR flunitrazepam)

6. TS = anti-anxiety agents

7. \#6 OR \#5 OR \#4

8. $\mathrm{TS}=$ (psychotherap OR incentive $\mathrm{OR}$ voucher OR psychosocial OR reinforcement OR motivation OR contingent OR advice OR biofeedback OR community OR education)

9. $\mathrm{TS}=$ (behavior AND therapy)

10. $\mathrm{TS}=$ psychotherapy

11. $\mathrm{TS}=$ (counselling OR counsel)

12. $\mathrm{TS}=$ cognitive therapy

13. $\mathrm{TS}=\mathrm{CBT}$

14. $\mathrm{TS}=$ brief intervention

15. $\mathrm{TS}=$ early intervention

16. $\mathrm{TS}=$ family therapy

17. $\mathrm{TS}=$ coping skill

18. $\mathrm{TS}=$ supportive expressive therapy

19. $\mathrm{TS}=$ social skill

20. $\mathrm{TS}=$ stress management

21. $\mathrm{TS}=$ social support

22. $\mathrm{TS}=$ relaxation therapy

23. $\mathrm{TS}=$ relapse prevention

24. $\mathrm{TS}=$ dialectical behaviour

25. $\mathrm{TS}=$ motivational interview

26. $\mathrm{TS}=$ motivational enhance

Psychosocial interventions for benzodiazepine harmful use, abuse or dependence (Review)

Copyright @ 2015 The Cochrane Collaboration. Published by John Wiley \& Sons, Ltd. 
28. $\mathrm{TS}=$ randomised controlled trial

29. $\mathrm{TS}=$ controlled clinical trial

30. $\mathrm{TS}=$ randomized

31. $\mathrm{TS}=$ placebo

32. $T S=$ drug therapy

33. $\mathrm{TS}=$ randomly

34. $\mathrm{TS}=$ trial

35. $\mathrm{TS}=$ groups

36. $\mathrm{TS}=$ (animals NOT humans)

37. \#35 OR \#34 OR\#33 OR \#32 OR\#31 OR\#30 OR\#29 OR\#28

38. \#37 NOT \#36

39. \#3 AND \#7 AND \#27 AND \#38

\section{Appendix 12. Dissertation Abstracts}

all(benzodiazepine*) OR all((chlordiazepoxide OR diazepam)) OR all((alprazolam OR lorazepam)) OR all((prazepam OR clobazam)) OR all((bromazepam OR flurazepam)) OR all((triazolam OR clonazepam)) OR all((temazepam OR nitrazepam)) OR all((lormetazepam OR flunitrazepam)) OR all(“Antianxiety Agents”) AND all(addict*) OR all((overdose* OR intoxicate*)) OR all((abstain* OR abstain)) OR all((withdraw* OR abus*)) OR all((abusing OR misuse*)) OR all((disorder* OR dependent*) OR all(polyabus*)

\section{Appendix 13. Index to Theses}

all(benzodiazepine* $)$ OR all((chlordiazepoxide OR diazepam)) OR all((alprazolam OR lorazepam)) OR all((prazepam OR clobazam)) OR all((bromazepam OR flurazepam)) OR all((triazolam OR clonazepam)) OR all((temazepam OR nitrazepam)) OR all((lormetazepam OR flunitrazepam)) OR all(“Antianxiety Agents”) AND all(addict*) OR all((overdos* OR intoxicat* )) OR all((abstin* OR abstain)) OR all((withdraw* OR abus* $)$ ) OR all((abusing OR misus*)) OR all((disorder* OR dependen*)) OR all(polyabus*)

\section{Appendix 14. 'Risk of bias' assessment tool}

Criteria for 'Risk of bias' assessment in RCTs (CDAG)

\begin{tabular}{ll|l}
\hline Item & Judgment & Description \\
\hline $\begin{array}{l}\text { 1. Random sequence generation (selection } \\
\text { bias) }\end{array}$ & Low risk & $\begin{array}{l}\text { The investigators describe a random component in the sequence gener- } \\
\text { ation process such as: random number table; computer random num- } \\
\text { ber generator; coin tossing; shuffling cards or envelopes; throwing dice; } \\
\text { drawing of lots; minimisation }\end{array}$ \\
\cline { 2 - 3 } & High risk & $\begin{array}{l}\text { The investigators describe a non-random component in the sequence } \\
\text { generation process such as: odd or even date of birth; date (or day) of } \\
\text { admission; hospital or clinic record number; alternation; judgement of } \\
\text { the clinician; results of a laboratory test or a series of tests; availability of } \\
\text { the intervention }\end{array}$ \\
\hline
\end{tabular}

Psychosocial interventions for benzodiazepine harmful use, abuse or dependence (Review) 
Unclear risk Insufficient information about the sequence generation process to permit judgement of low or high risk

2. Allocation concealment (selection bias) Low risk

Investigators enrolling participants could not foresee assignment because one of the following, or an equivalent method, was used to conceal allocation: central allocation (including telephone, web-based, and pharmacycontrolled, randomisation); sequentially numbered drug containers of identical appearance; sequentially numbered, opaque, sealed envelopes

High risk Investigators enrolling participants could possibly foresee assignments because one of the following method was used: open random allocation schedule (e.g. a list of random numbers); assignment envelopes without appropriate safeguards (e.g. if envelopes were unsealed or nonopaque or not sequentially numbered); alternation or rotation; date of birth; case record number; any other explicitly unconcealed procedure

Unclear risk Insufficient information to permit judgement of low or high risk. This is usually the case if the method of concealment is not described or not described in sufficient detail to allow a definite judgement

3. Blinding of participants and providers Low risk (performance bias)

No blinding or incomplete blinding, but the review authors judge that the outcome is not likely to be influenced by lack of blinding; blinding of participants and key study personnel ensured, and unlikely that the blinding could have been broken

High risk No blinding or incomplete blinding, and the outcome is likely to be influenced by lack of blinding; blinding of key study participants and personnel attempted, but likely that the blinding could have been broken, and the outcome is likely to be influenced by lack of blinding

Unclear risk Insufficient information to permit judgement of low or high risk

4. Blinding of participants and providers Low risk (performance bias)

Subjective outcomes

Blinding of participants and providers and unlikely that the blinding could have been broken

High risk

No blinding or incomplete blinding, and the outcome is likely to be influenced by lack of blinding; blinding of key study participants and personnel attempted, but likely that the blinding could have been broken, and the outcome is likely to be influenced by lack of blinding

Unclear risk Insufficient information to permit judgement of low or high risk

5. Blinding of outcome assessor (detection Low risk bias)

Objective outcomes
No blinding of outcome assessment, but the review authors judge that the outcome measurement is not likely to be influenced by lack of blinding; blinding of outcome assessment ensured, and unlikely that the blinding could have been broken 
High risk No blinding of outcome assessment, and the outcome measurement is likely to be influenced by lack of blinding; blinding of outcome assessment, but likely that the blinding could have been broken, and the outcome measurement is likely to be influenced by lack of blinding

Unclear risk Insufficient information to permit judgement of low or high risk

6. Blinding of outcome assessor (detection Low risk bias)

Subjective outcomes
No blinding of outcome assessment, but the review authors judge that the outcome measurement is not likely to be influenced by lack of blinding; blinding of outcome assessment ensured, and unlikely that the blinding could have been broken

High risk No blinding of outcome assessment, and the outcome measurement is likely to be influenced by lack of blinding; blinding of outcome assessment, but likely that the blinding could have been broken, and the outcome measurement is likely to be influenced by lack of blinding

Unclear risk Insufficient information to permit judgement of low or high risk

7. Incomplete outcome data (attrition bias) Low risk For all outcomes except retention in treatment or drop-out

No missing outcome data; reasons for missing outcome data unlikely to be related to true outcome (for survival data, censoring unlikely to be introducing bias); missing outcome data balanced in numbers across intervention groups, with similar reasons for missing data across groups; for dichotomous outcome data, the proportion of missing outcomes compared with observed event risk not enough to have a clinically relevant impact on the intervention effect estimate; for continuous outcome data, plausible effect size (difference in means or standardized difference in means) among missing outcomes not enough to have a clinically relevant impact on observed effect size; missing data have been imputed using appropriate methods; all randomised patients are reported/analysed in the group they were allocated to by randomisation irrespective of noncompliance and co-interventions (ITT)

High risk Reason for missing outcome data likely to be related to true outcome, with either imbalance in numbers or reasons for missing data across intervention groups; for dichotomous outcome data, the proportion of missing outcomes compared with observed event risk enough to induce clinically relevant bias in intervention effect estimate; for continuous outcome data, plausible effect size (difference in means or standardized difference in means) among missing outcomes enough to induce clinically relevant bias in observed effect size; 'as-treated' analysis done with substantial departure of the intervention received from that assigned at randomisation

Unclear risk Insufficient information to permit judgement of low or high risk (e.g. number randomised not stated, no reasons for missing data provided; number of drop-out not reported for each group) 
8. Selective reporting (reporting bias) Low risk The study protocol is available and all of the study's pre-specified (primary and secondary) outcomes that are of interest in the review have been reported in the pre-specified way; the study protocol is not available but it is clear that the published reports include all expected outcomes, including those that were pre-specified (convincing text of this nature may be uncommon)

High risk Not all of the study's pre-specified primary outcomes have been reported; one or more primary outcomes are reported using measurements, analysis methods or subsets of the data (e.g. subscales) that were not pre-specified; one or more reported primary outcomes were not pre-specified (unless clear justification for their reporting is provided, such as an unexpected adverse effect); one or more outcomes of interest in the review are reported incompletely so that they cannot be entered in a meta-analysis; the study report fails to include results for a key outcome that would be expected to have been reported for such a study

Unclear risk Insufficient information to permit judgement of low or high risk

\section{CONTRIBUTIONSOFAUTHORS}

All review authors drafted the protocol.

Dr. Catherine Darker developed and ran the search strategy.

Dr. Catherine Darker obtained the full-text of studies.

Dr. Catherine Darker, Dr. Brion Sweeney and Dr. Joe Barry selected which studies to include.

Dr. Catherine Darker, Dr. Joe Barry and Dr. Brion Sweeney extracted data from included studies.

Dr. Catherine Darker and Dr Erica Donnelly-Swift entered data into RevMan 2014.

Dr. Catherine Darker \& Dr Erica Donnelly-Swift performed the analyses.

All review authors interpreted the analyses.

All review authors (Dr. Catherine Darker conducted first draft) drafted the final review.

All review authors will update the review. 


\section{DECLARATIONSOF INTEREST}

Each review author (CD, BS, JB, MF, EDS) have no interests to declare relating to this work. This study was funded in part by a Cochrane Fellowship from the Health Research Board in Ireland to one of the review authors (CD).

\section{SOURCES OF SUPPORT}

\section{Internal sources}

- Department of Public Health \& Primary Care, Trinity College Dublin, Ireland.

- Tallaght Hospital Library, Tallaght Hospital, Dublin 24, Ireland.

\section{External sources}

- Cochrane Fellowship Funding, Health Research Board (HRB), Ireland.

\section{DIFFERENCES BETWEEN PROTOCOLAND REVIEW}

There are four differences between the protocol, Darker 2012, and this Cochrane review. First, we excluded controlled clinical trials and this review now includes RCTs only. Secondly, we excluded data relating to z-hypnotics. Thirdly, we included two additional outcomes in the review - reduction of BZDs by $>50 \%$ as an additional outcome of interest and drop-outs/loss to follow-up. Fourthly, due to the complexity of the systematic review and subsequent meta-analyses, we deemed that it was beyond the scope of this review to examine a number of secondary outcomes originally identified in the protocol, such as craving, mortality, deliberate self-harm, quality of life, legal problems/crime, physical health, psychological health, adverse outcomes, health service usage and severity of dependence. 